\title{
Total Synthesis and Structural Revision of Cyclotetrapeptide Asperterrestide A
}

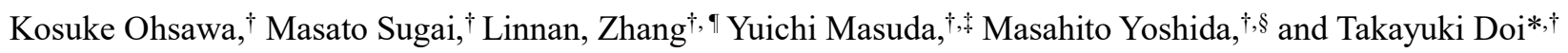

$\dagger^{\dagger}$ Graduate School of Pharmaceutical Sciences, Tohoku University, 6-3 Aza-aoba, Aramaki, Aoba-ku, Sendai, 980-8578, Japan

*Corresponding author

E-mail address: doi_taka@mail.pharm.tohoku.ac.jp

Phone number: +81-22-795-6865

Fax number $+81-22-795-6864$

ORCID: Takayuki Doi 0000-0002-8306-6819

Present address

${ }^{\natural}$ Faculty of Pharmaceutical Engineering, Shenyang Pharmaceutical University, No.26 Huatuo Rd, High \& New Tech Development Zone, Benxi, Liaoning, 117004, P.R.China

$\$$ Graduate School of Bioresources, Mie university, 1577 Kurimamachiya-cho, Tsu, 514-8507, Japan

$\S$ Department of Chemistry, Graduate School of Pure and Applied Sciences, University of Tsukuba, 1-1-1 Tennodai, Tsukuba, 305-8571, Japan 


\section{Table of Contents}

1. ${ }^{1} \mathrm{H}$ and ${ }^{13} \mathrm{C}$ NMR spectra of synthetic compounds $\quad$ S3-S52

2. NOESY spectra of synthetic $\mathbf{1 a}$ and $\mathbf{1 b} \quad$ S53-S54

3. Comparisons of NMR spectroscopic data observed in $\mathrm{CDCl}_{3}$ between natural asperterrestide A, synthetic 1a and 1b $\quad$ S55-S58

4. Cartesian coordinates of the conformers of $\mathbf{1 d}$

5. Cartesian coordinates of the conformers of $\mathbf{1 e}$

S59-S62

6. Cartesian coordinates of the conformers of $\mathbf{1 f}$

S63-S65

7. Correlation between observed NMR spectroscopic data and calculated values by molecular modeling

S66-S69

S70-S61

8. A panel of three selected cancer cell lines for cytotoxicity

S72-S74 


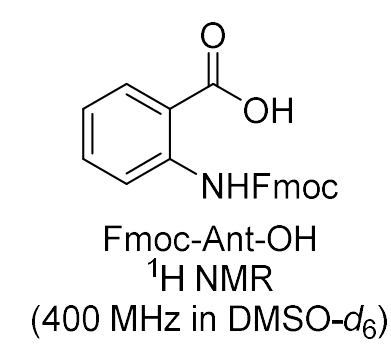

${ }^{1} \mathrm{H}$ NMR spectrum of Fmoc-Ant-OH

$(400 \mathrm{MHz}$ in DMSO-d

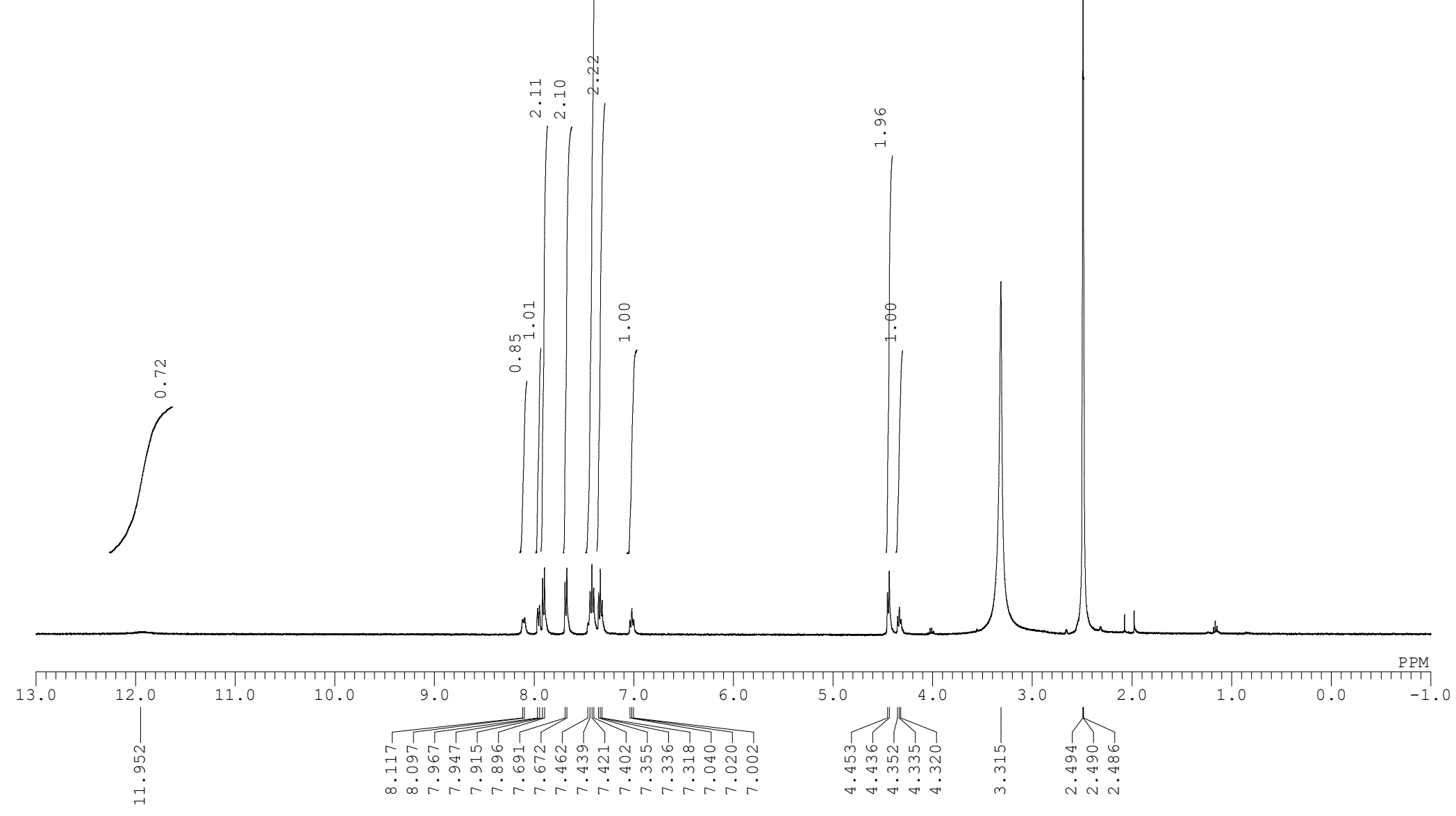




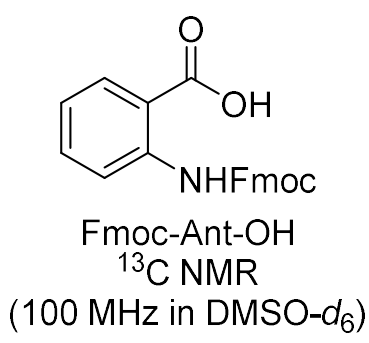

${ }^{13} \mathrm{C}$ NMR spectrum of Fmoc-Ant-OH

$\left(100 \mathrm{MHz}\right.$ in DMSO-d $\left.{ }_{6}\right)$
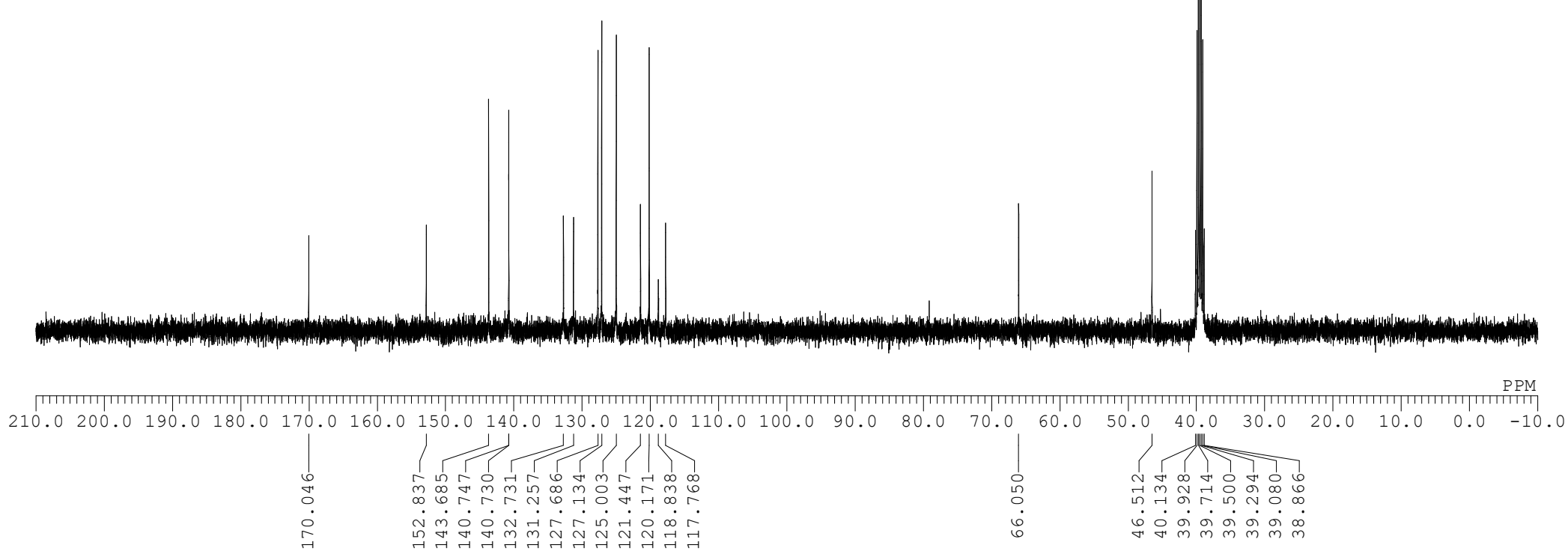
TBSO

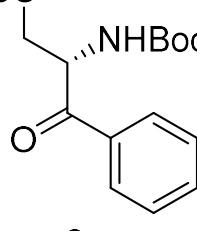

3

${ }^{1} \mathrm{H}$ NMR

(400 $\mathrm{MHz}$ in $\mathrm{CDCl}_{3}$ )

${ }^{1} \mathrm{H}$ NMR spectrum of $\mathbf{3}$

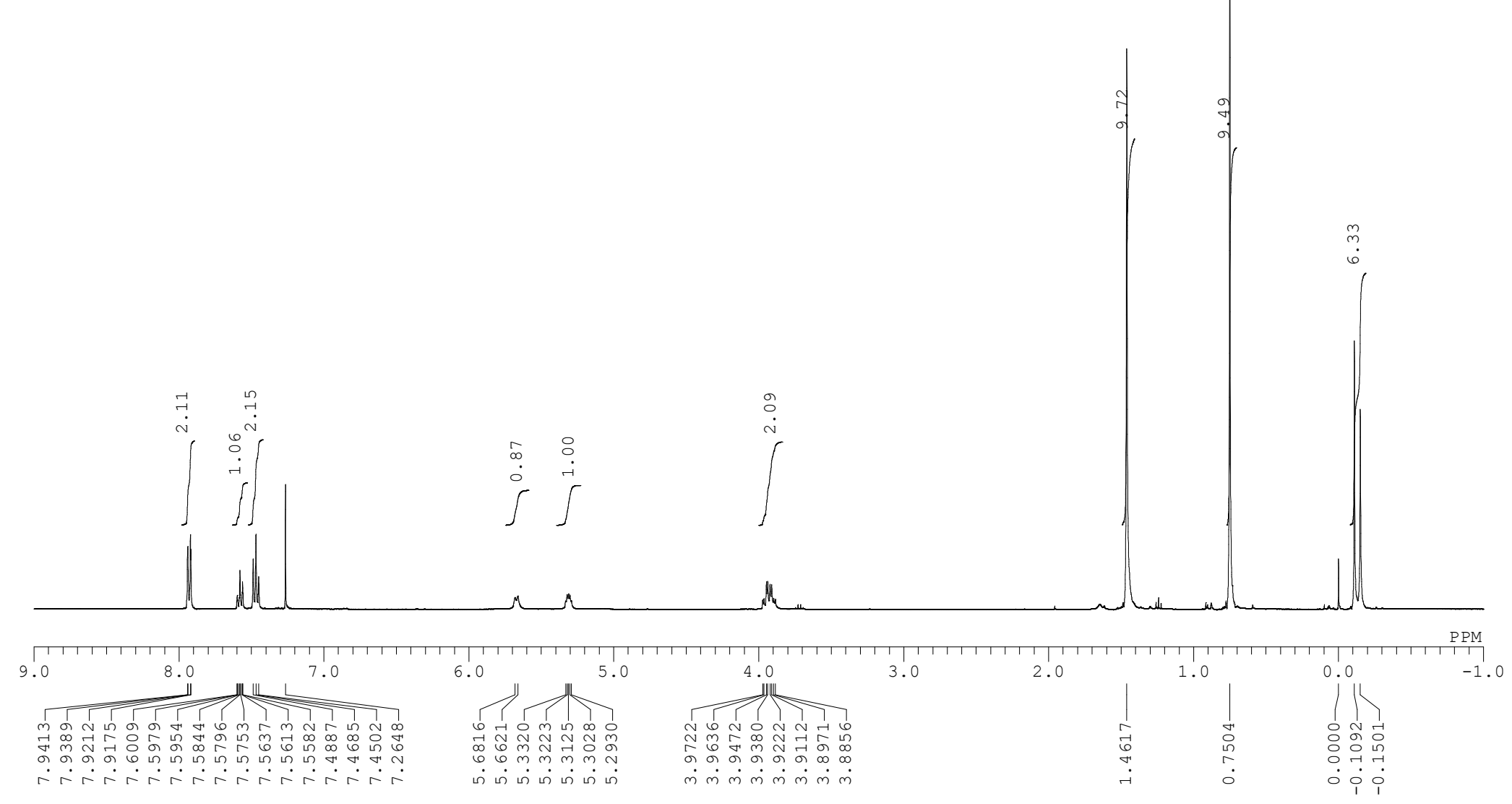


TBSO

NNHBoc

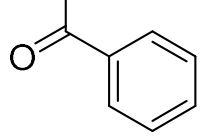

3

${ }^{13} \mathrm{C}$ NMR

(100 $\mathrm{MHz}$ in $\mathrm{CDCl}_{3}$ )
${ }^{13} \mathrm{C}$ NMR spectrum of $\mathbf{3}$

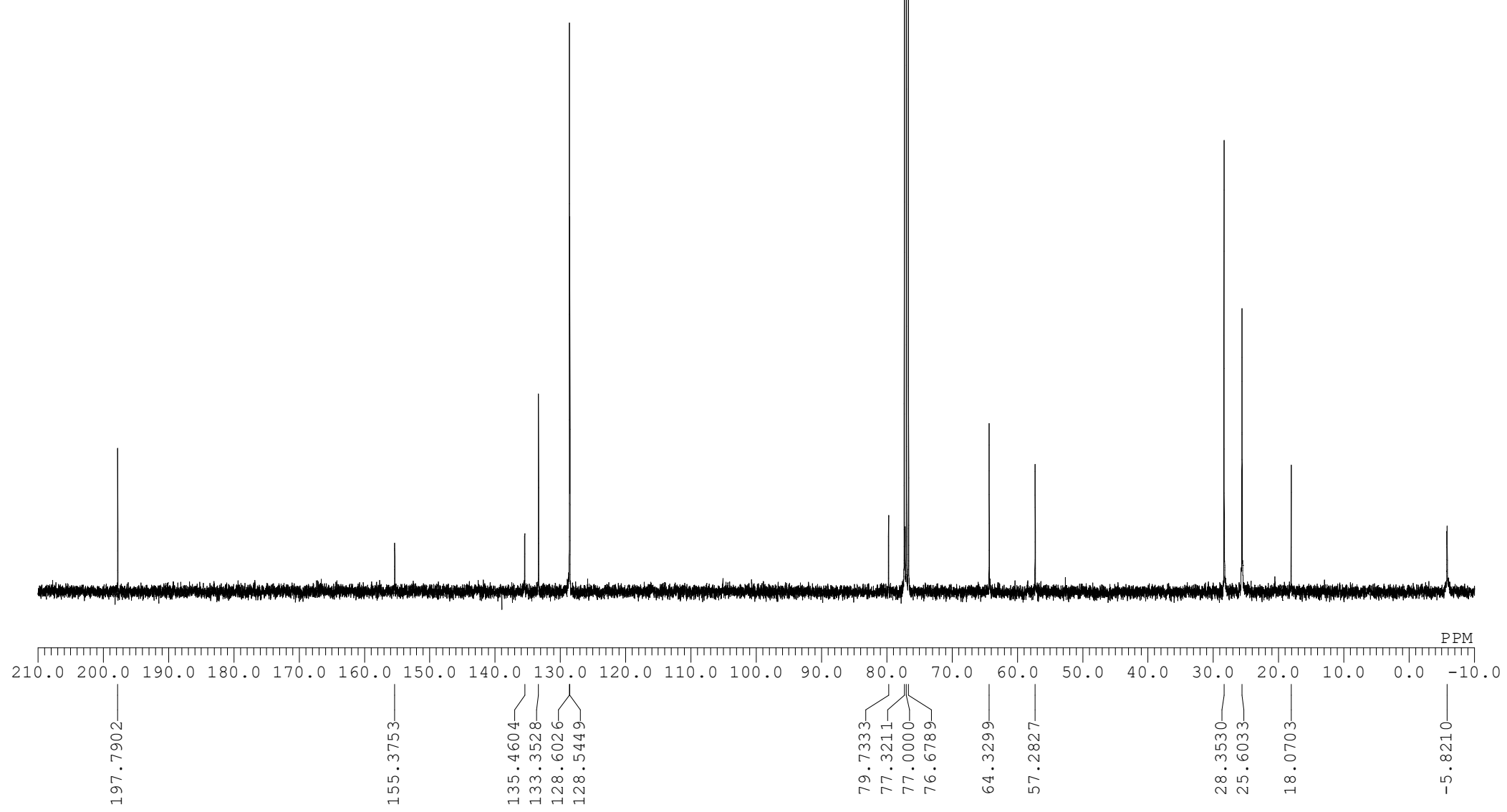


TBSO

$\mathrm{HO}$

$4 a$

${ }^{1} \mathrm{H}$ NMR

(400 MHz in $\mathrm{CDCl}_{3}$ )

including diastreomer

NHBoc<smiles>[c]1ccccc1</smiles>

${ }^{1} \mathrm{H}$ NMR spectrum of $\mathbf{4 a}$ 
TBSO

$\mathrm{HO}$

NHBoc

$4 a$

${ }^{13} \mathrm{C}$ NMR

$\left(100 \mathrm{MHz}\right.$ in $\mathrm{CDCl}_{3}$ )

including diastreomer
${ }^{13} \mathrm{C}$ NMR spectrum of $\mathbf{4 a}$

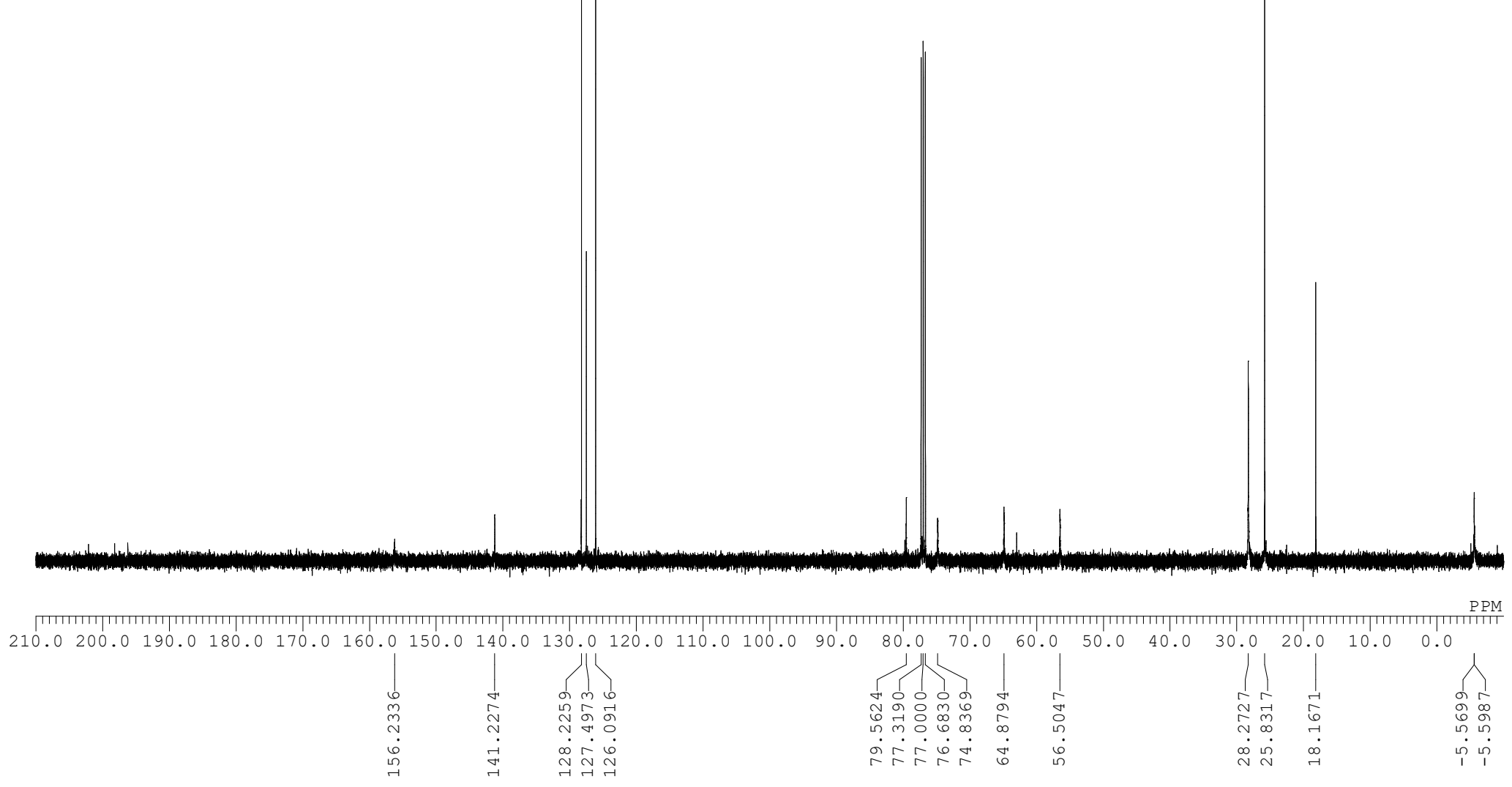


TBSO

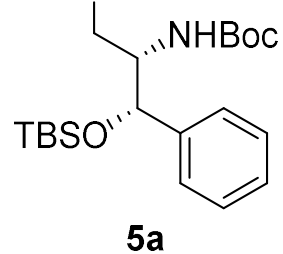

HNMR

(400 MHz in $\mathrm{CDCl}_{3}$ )

including diastreomer
${ }^{1} \mathrm{H}$ NMR spectrum of $\mathbf{5 a}$

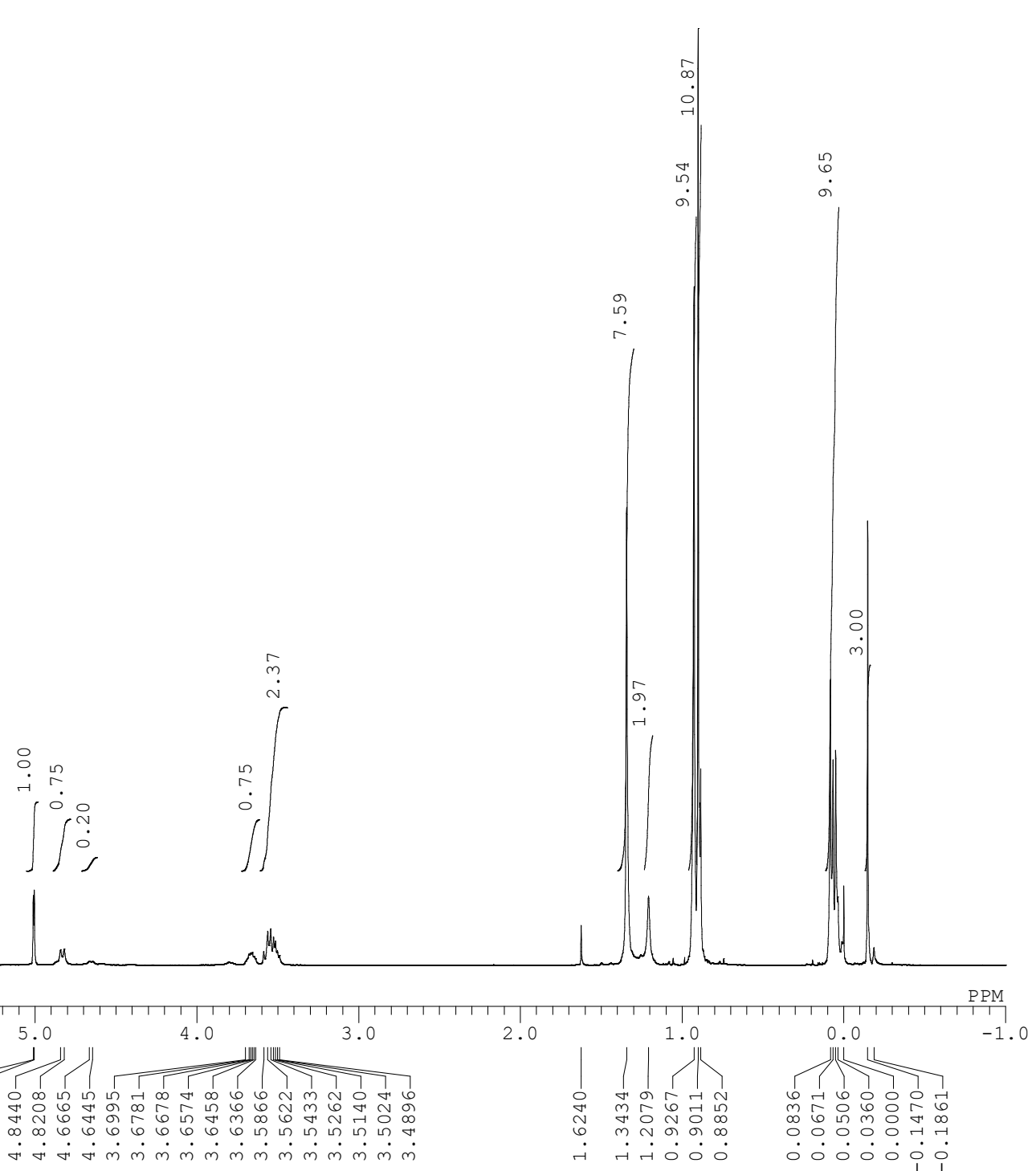


TBSO

NHBoc

TBSO"

5 a

${ }^{13} \mathrm{C}$ NMR

$\left(100 \mathrm{MHz}\right.$ in $\left.\mathrm{CDCl}_{3}\right)$

including diastreomer
${ }^{13} \mathrm{C}$ NMR spectrum of $\mathbf{5 a}$

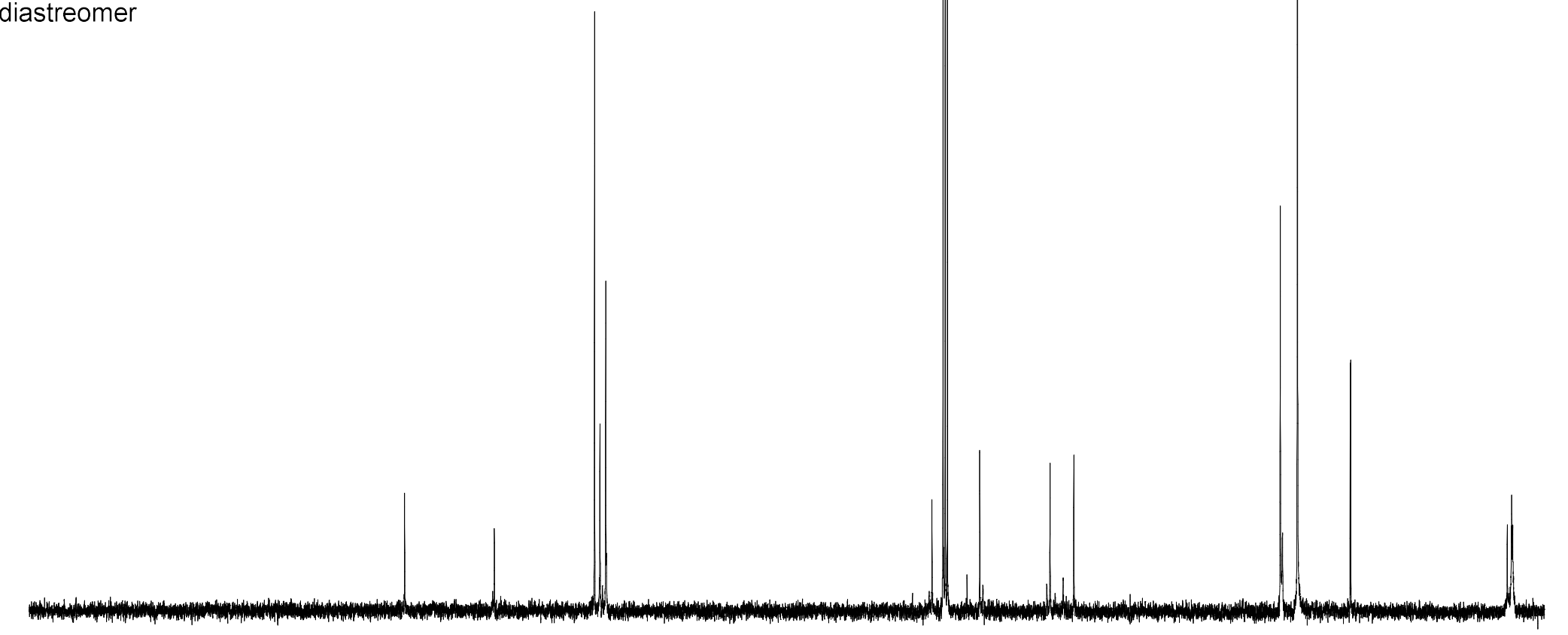

पPM $210.0200 .0190 .0 \quad 180.0170 .0 \quad 160.0150 .0140 .0130 .0120 .0110 .0100 .090 .60$
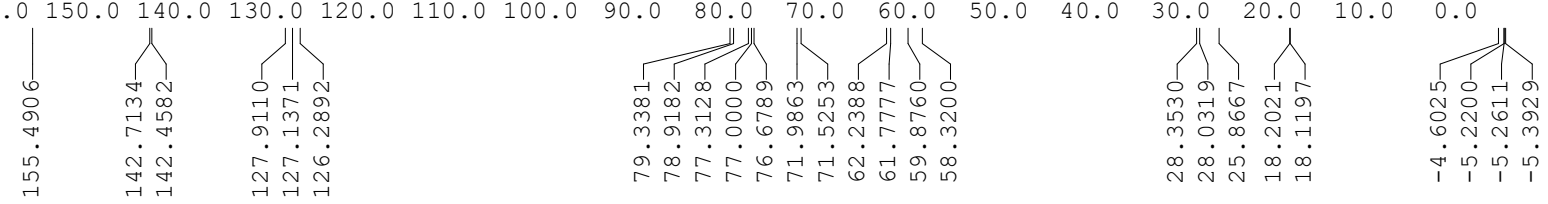


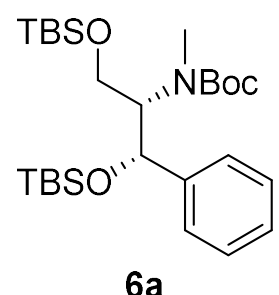

${ }^{1} \mathrm{H}$ NMR spectrum of $\mathbf{6 a}$

${ }^{1} \mathrm{H} N M R$

(400 MHz in $\mathrm{CDCl}_{3}$ )

including diastreomer

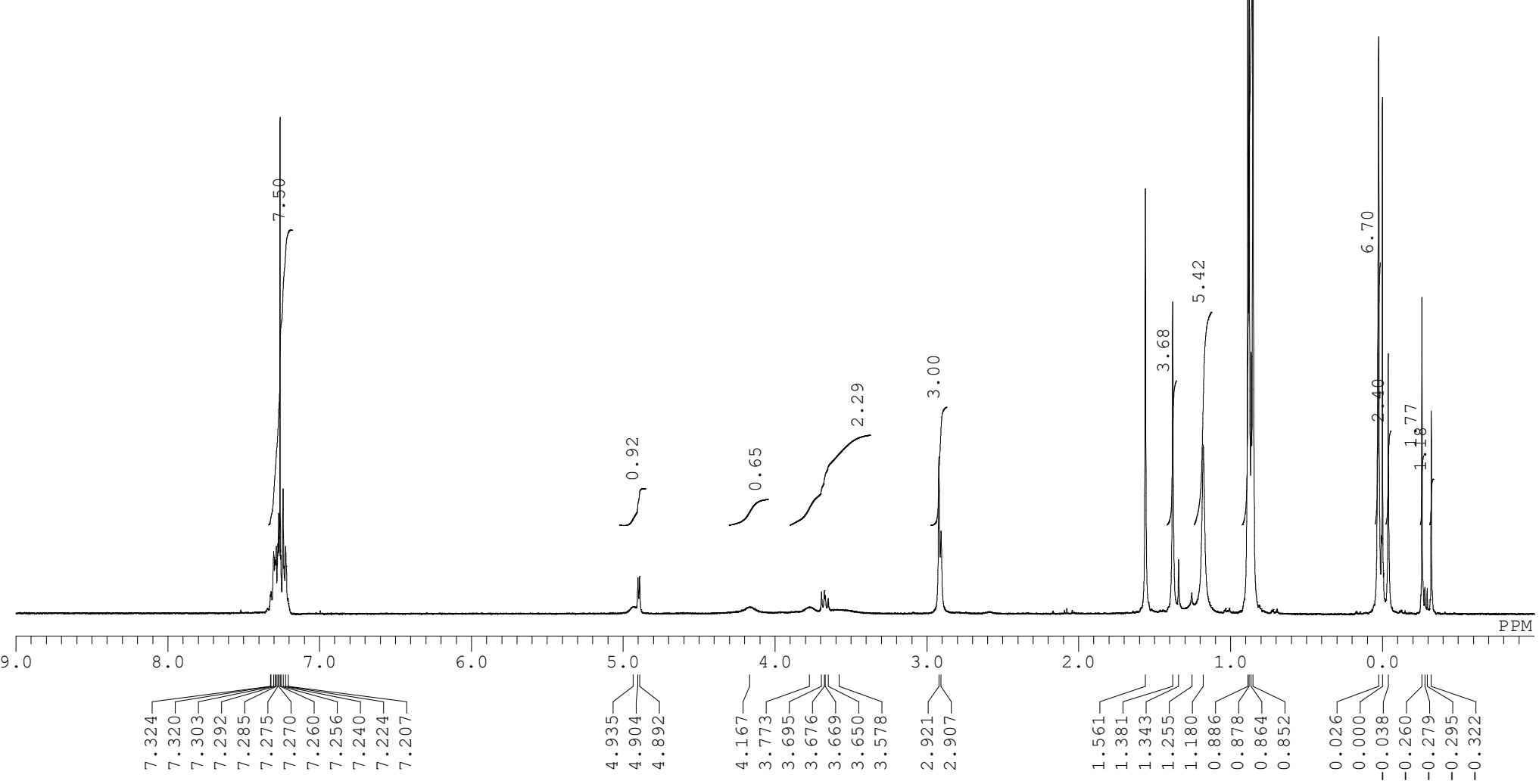




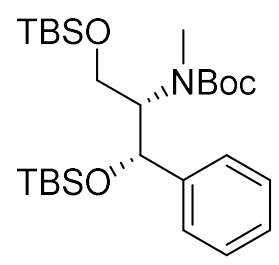

${ }^{13} \mathrm{C}$ NMR spectrum of $\mathbf{6 a}$

$6 a$

${ }^{13} \mathrm{C}$ NMR

(100 $\mathrm{MHz}$ in $\mathrm{CDCl}_{3}$ )

including diastreomer

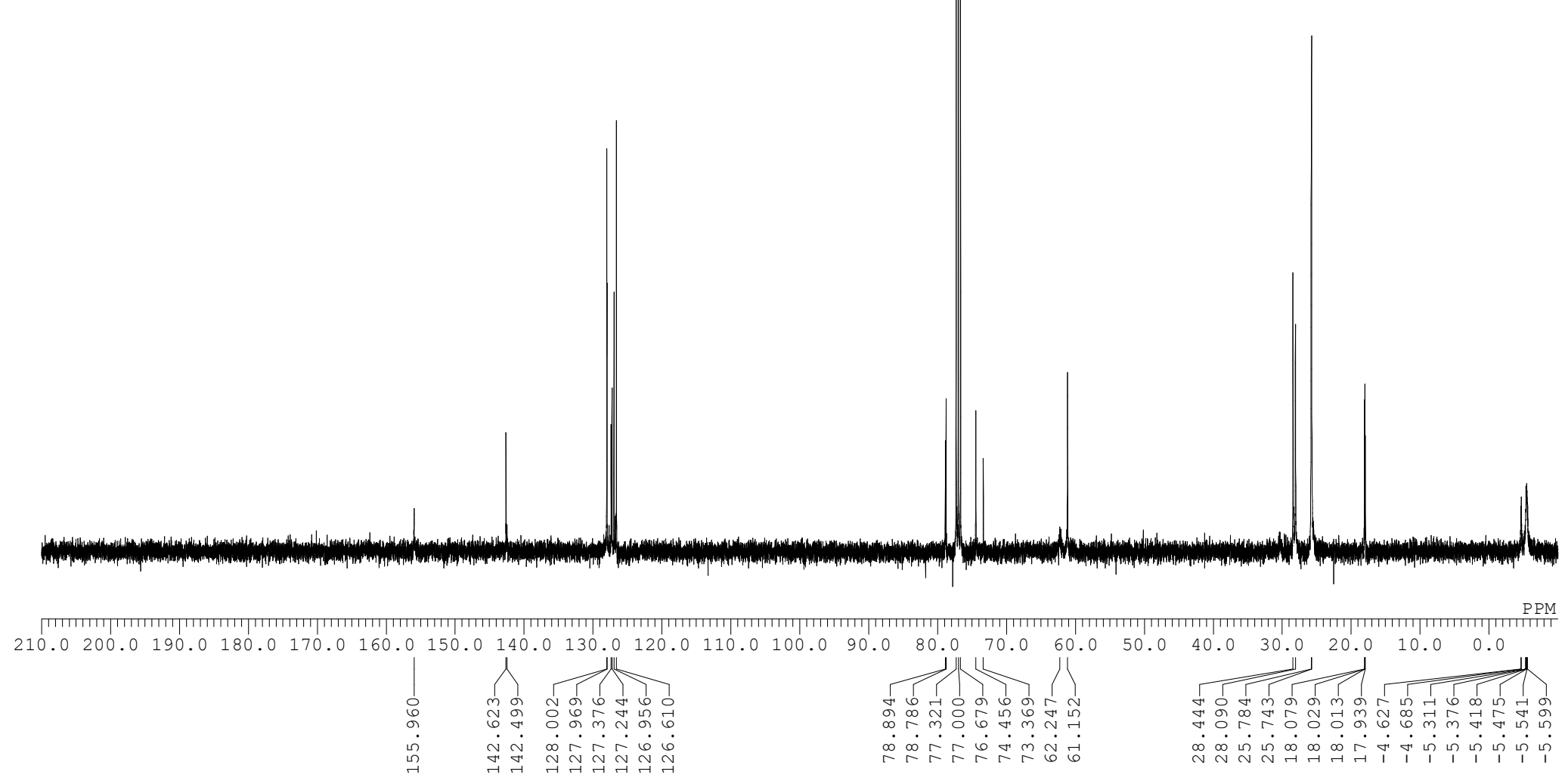




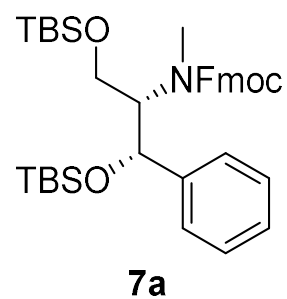

${ }^{1} \mathrm{H}$ NMR spectrum of $7 \mathbf{a}$

${ }^{1} \mathrm{H}$ NMR

(400 $\mathrm{MHz}$ in $\mathrm{CDCl}_{3}$ )

including diastreomer
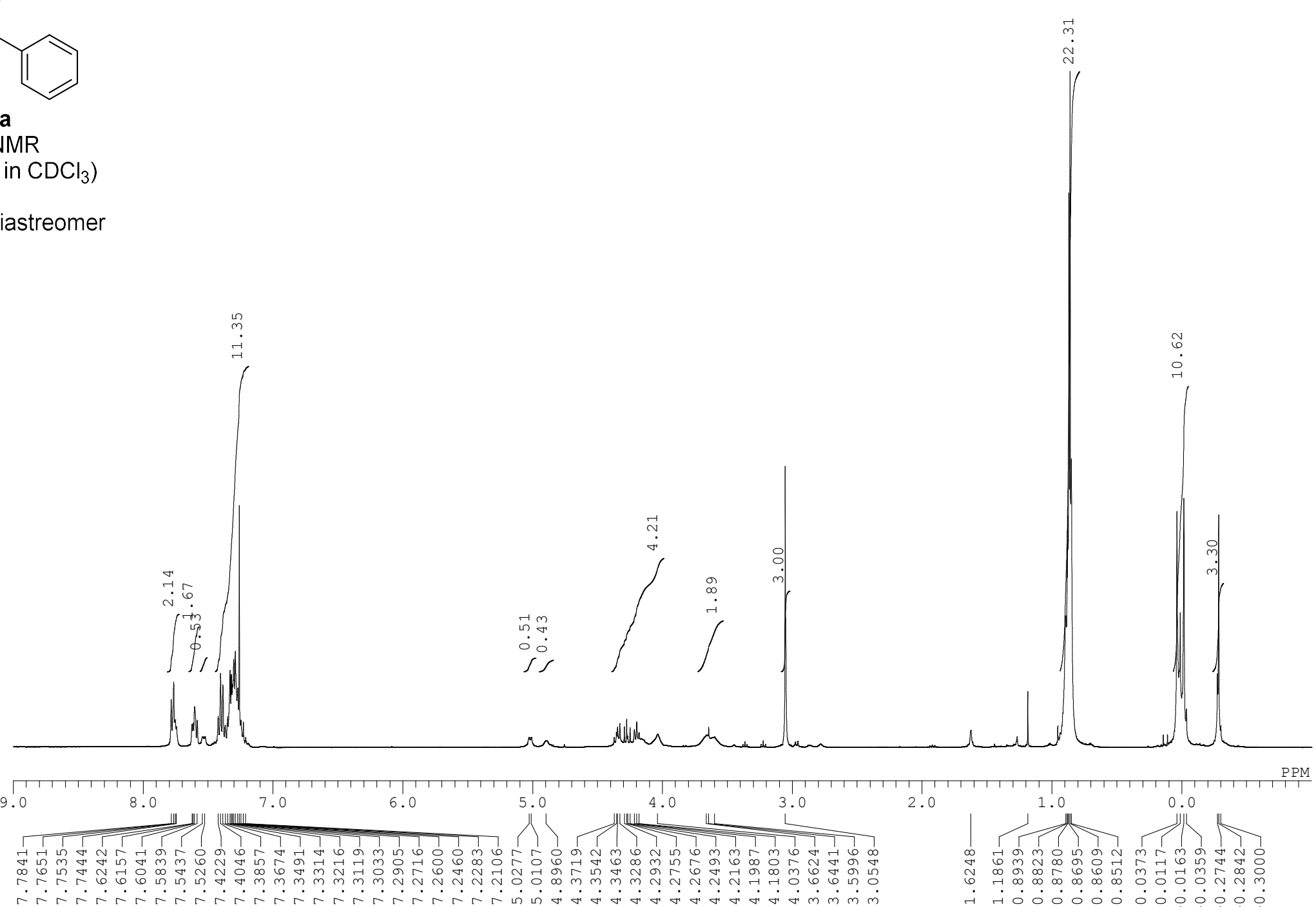

2.0

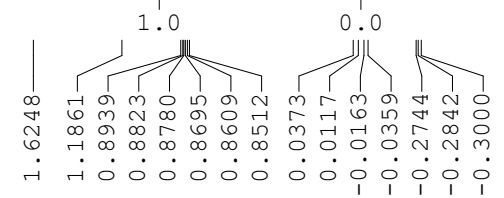




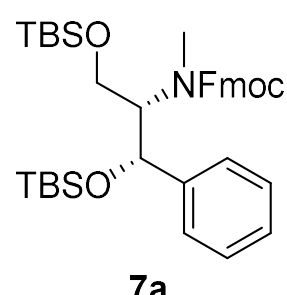

$7 a$

${ }^{13} \mathrm{C}$ NMR

(100 MHz in $\mathrm{CDCl}_{3}$ )

including diastreomer

${ }^{13} \mathrm{C}$ NMR spectrum of $7 \mathbf{a}$

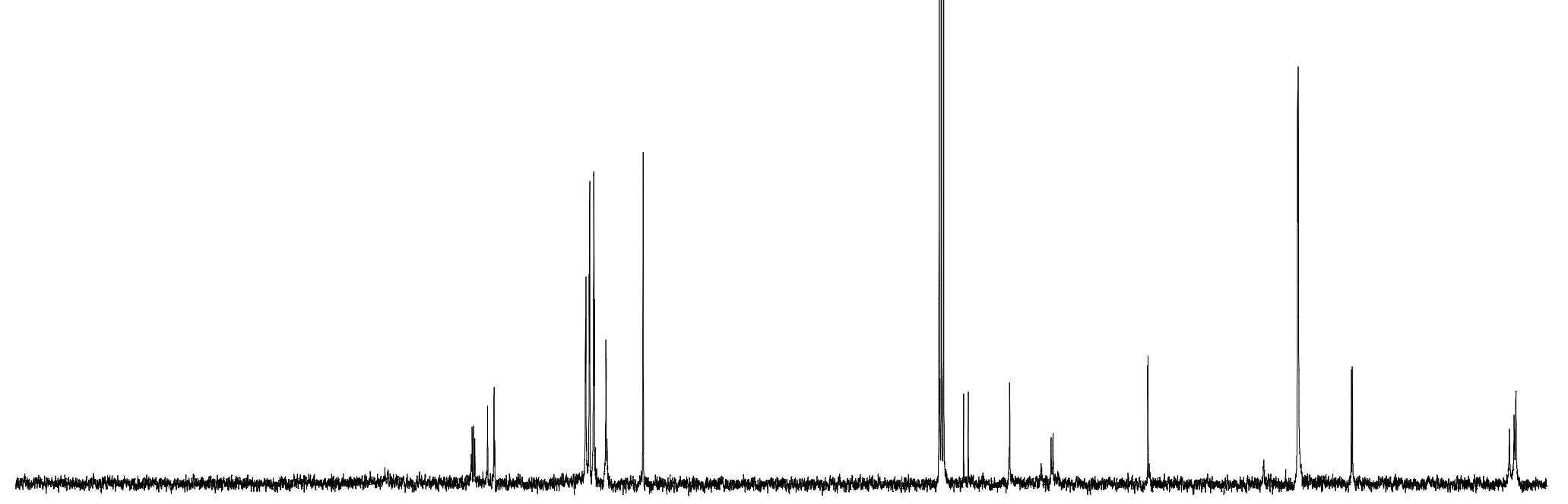

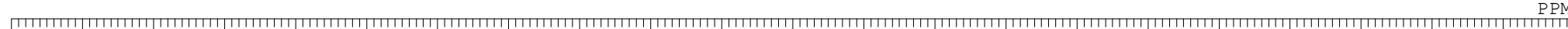

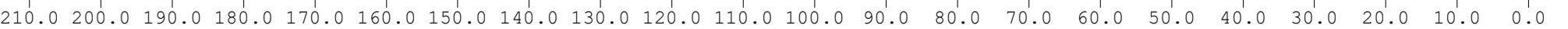
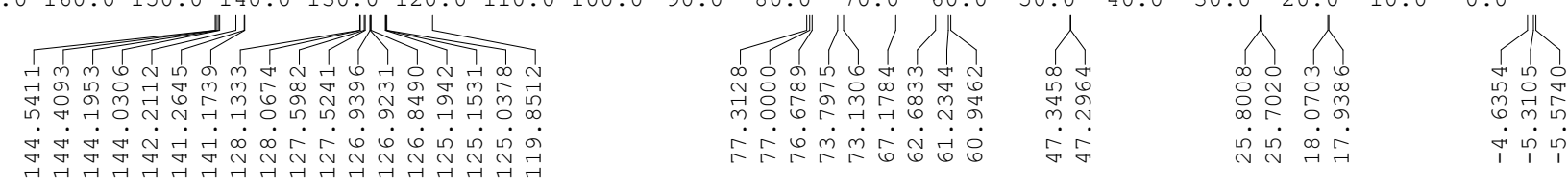


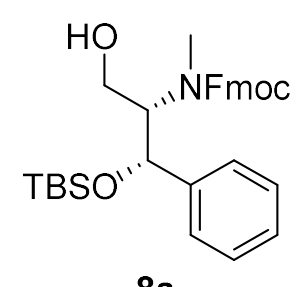

${ }^{1} \mathrm{H}$ NMR spectrum of $\mathbf{8 a}$

${ }^{1} \mathrm{H}$ NMR

(400 $\mathrm{MHz}$ in $\mathrm{CDCl}_{3}$ )
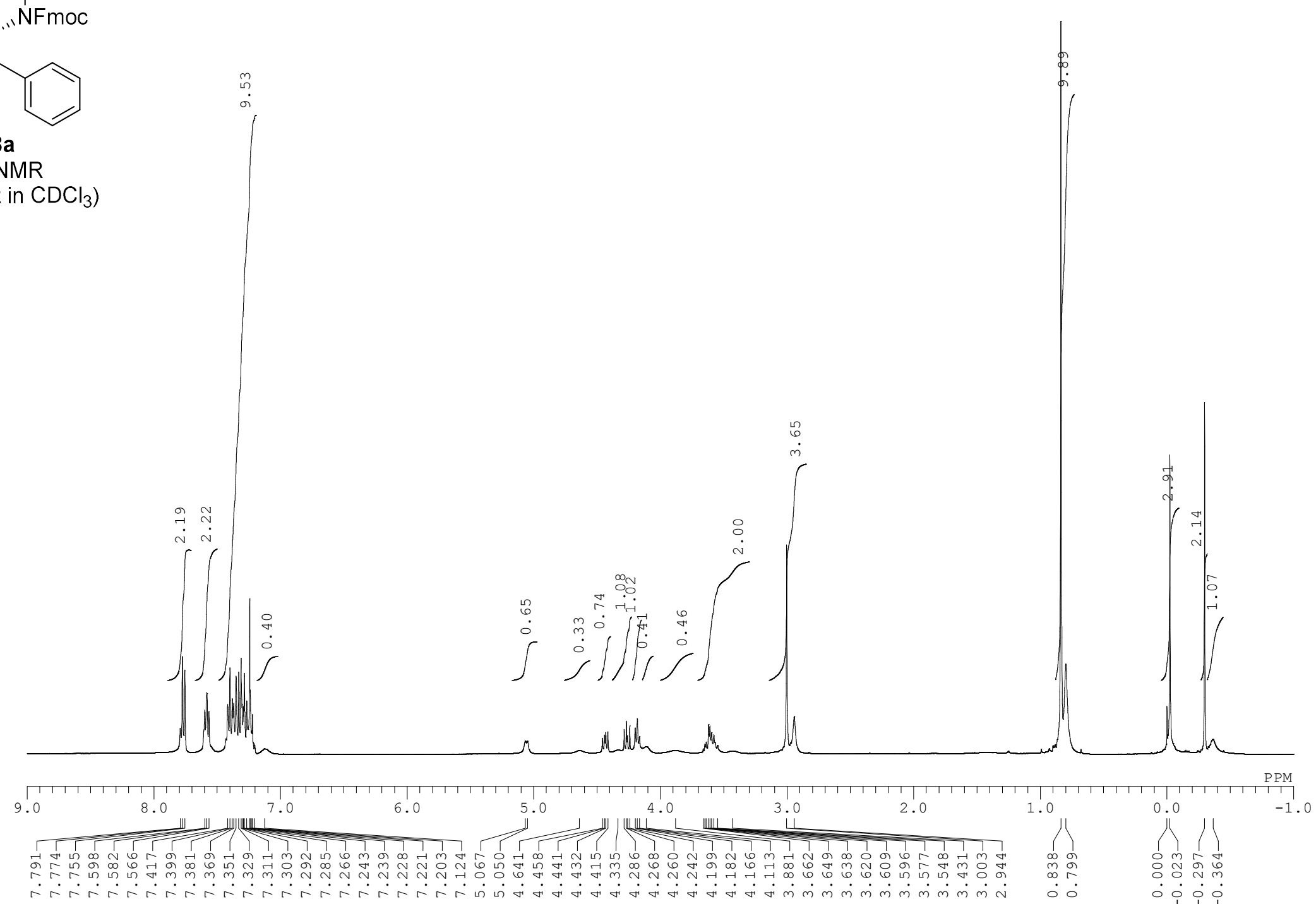


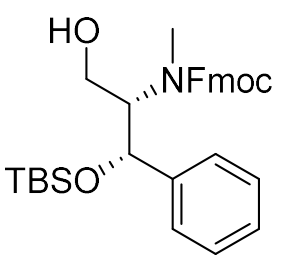

${ }^{13} \mathrm{C}$ NMR spectrum of $\mathbf{8 a}$

8

${ }^{13} \mathrm{C}$ NMR

$\left(100 \mathrm{MHz}\right.$ in $\left.\mathrm{CDCl}_{3}\right)$

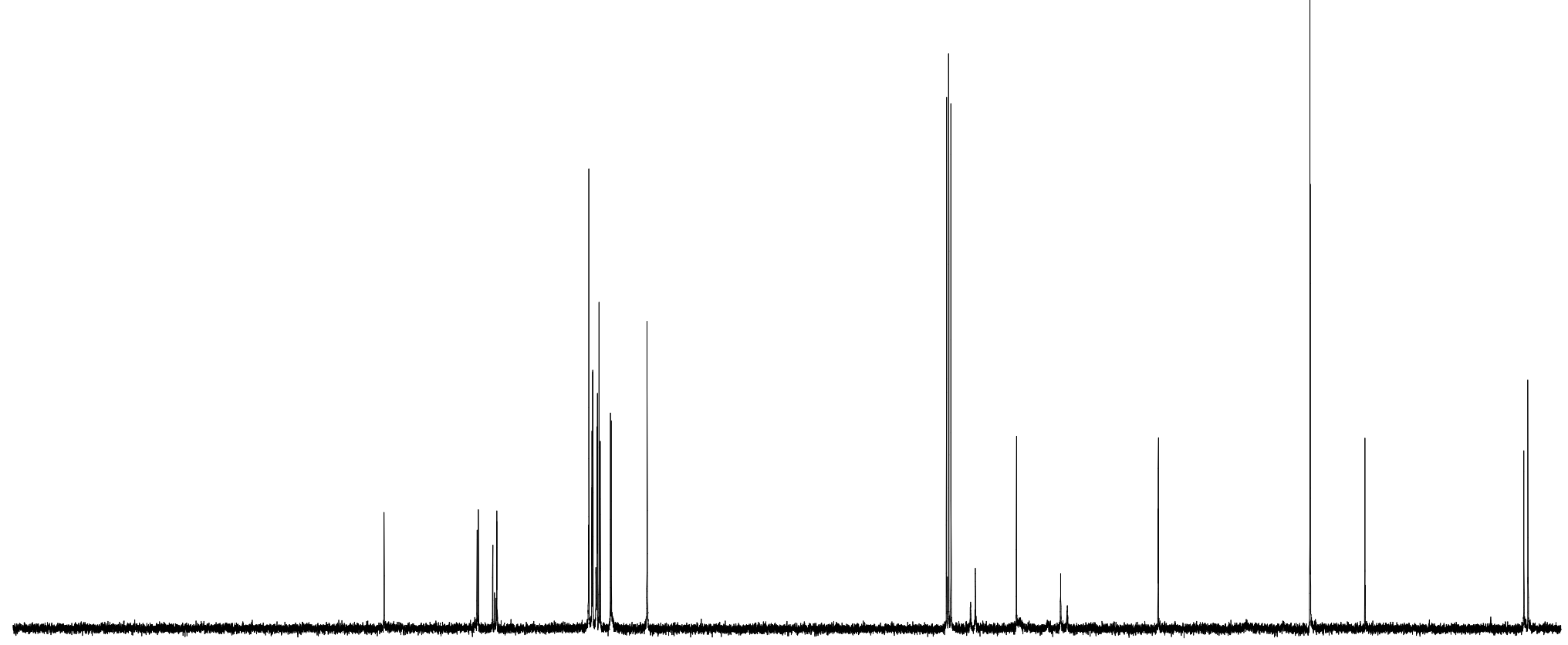

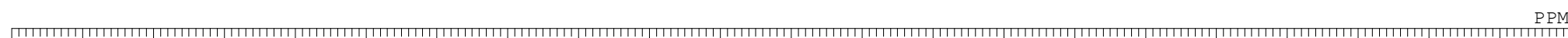

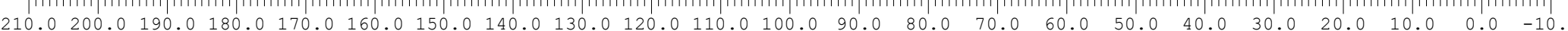
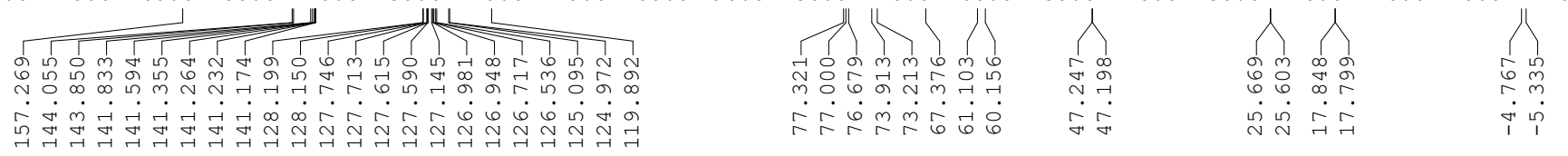


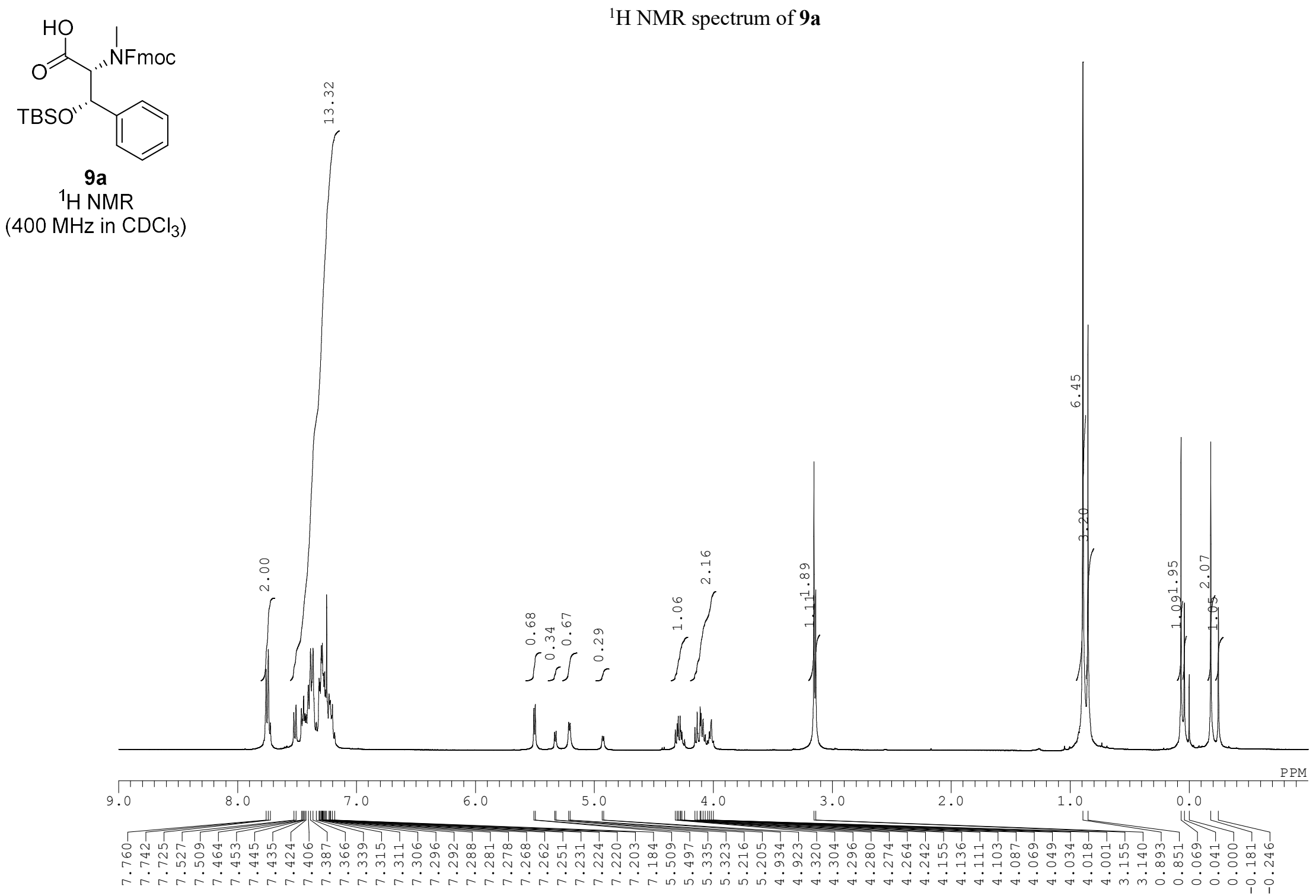




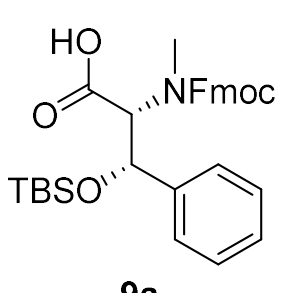

.

${ }^{13} \mathrm{C}$ NMR

$\left(100 \mathrm{MHz}\right.$ in $\left.\mathrm{CDCl}_{3}\right)$

${ }^{13} \mathrm{C}$ NMR spectrum of $\mathbf{9 a}$

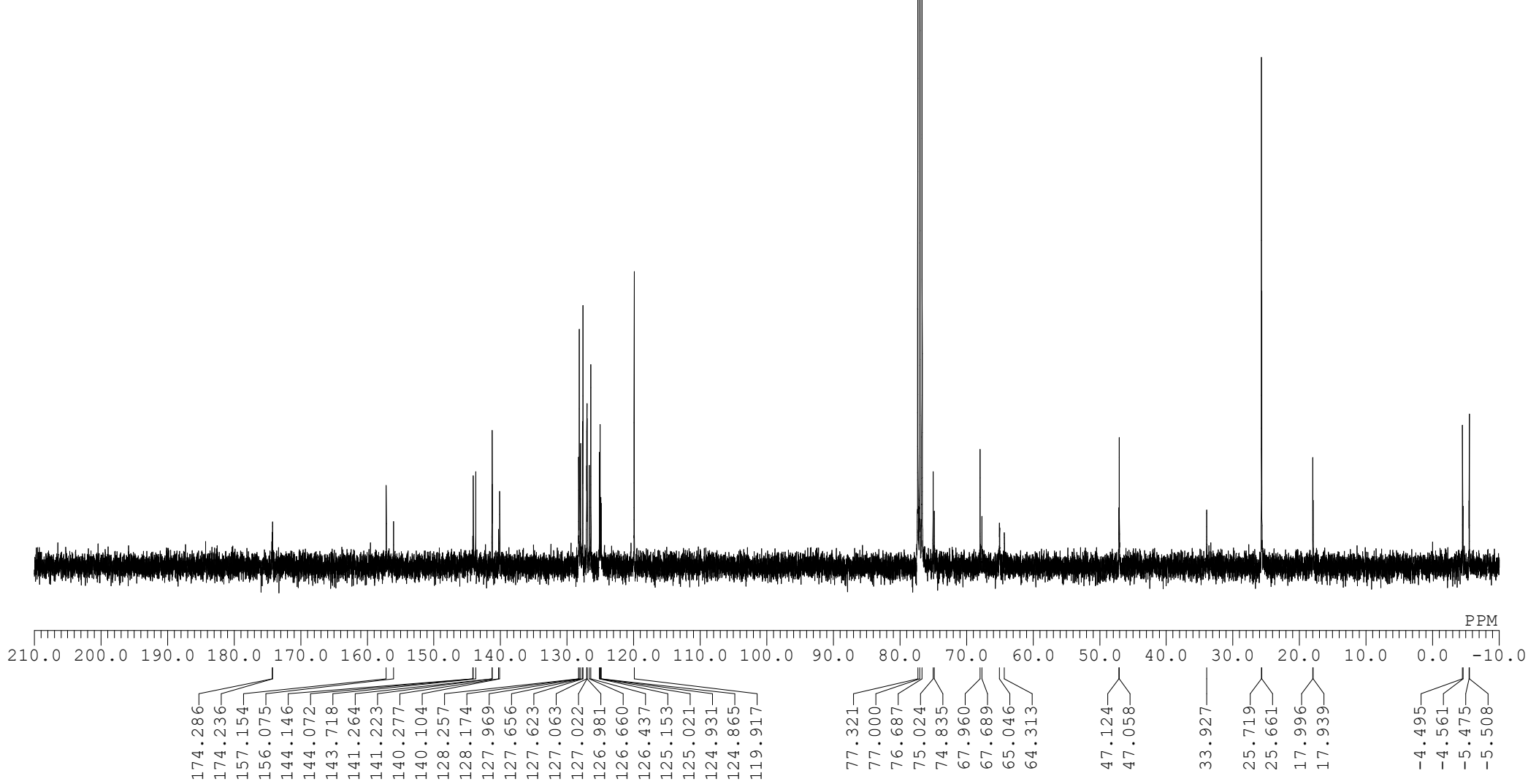




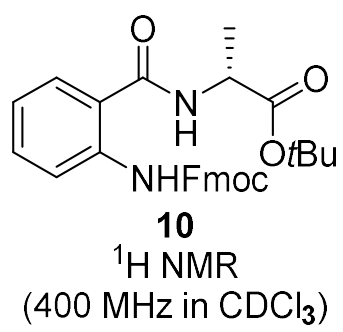

${ }^{1} \mathrm{H}$ NMR spectrum of $\mathbf{1 0}$

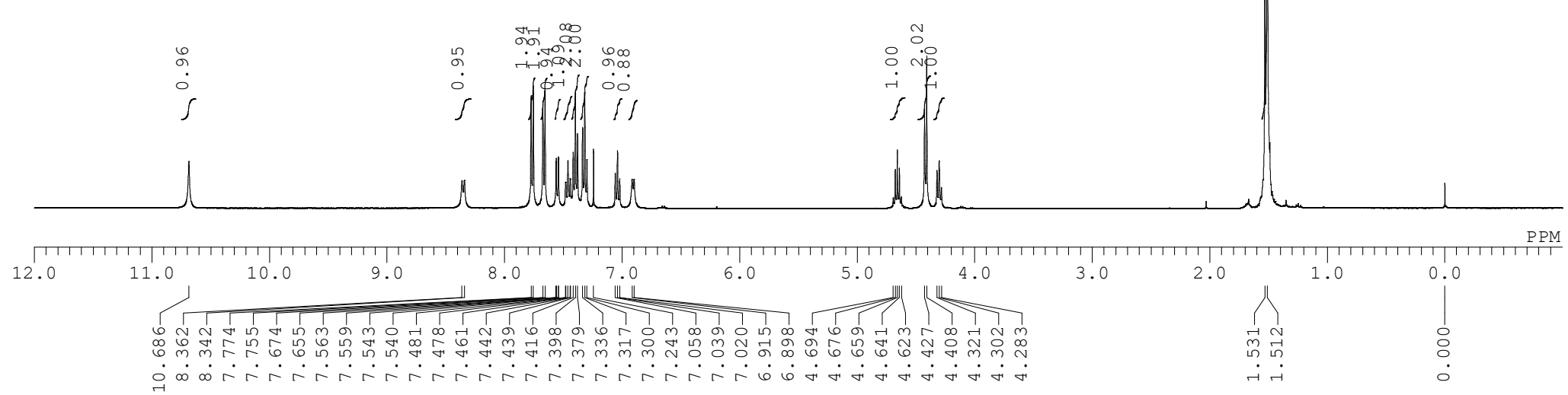




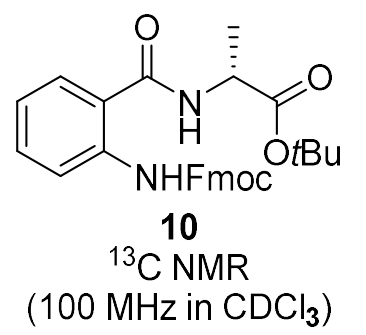

${ }^{13} \mathrm{C}$ NMR spectrum of $\mathbf{1 0}$

$\left(100 \mathrm{MHz}\right.$ in $\left.\mathrm{CDCl}_{3}\right)$

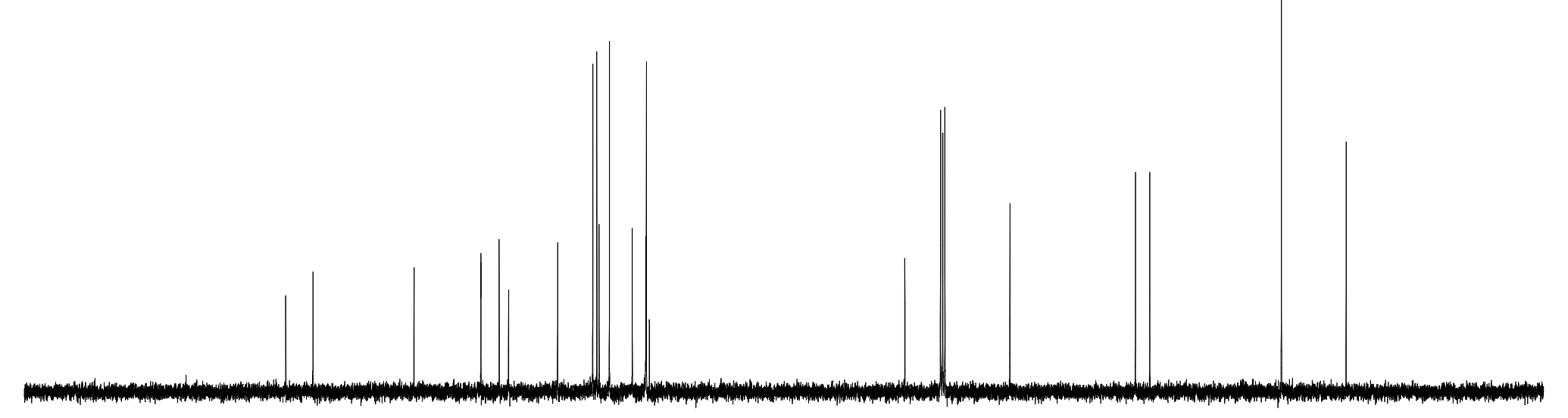

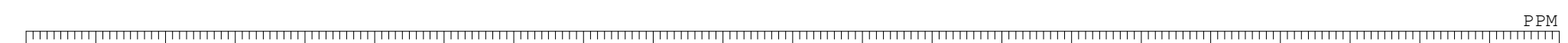

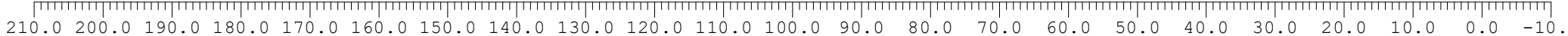

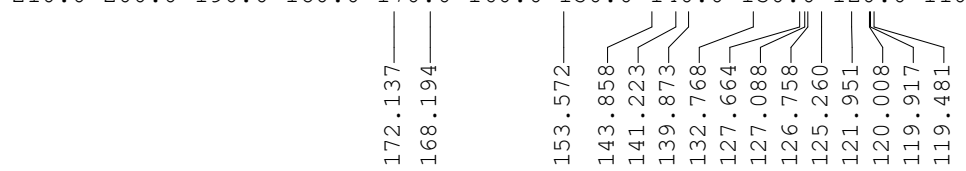

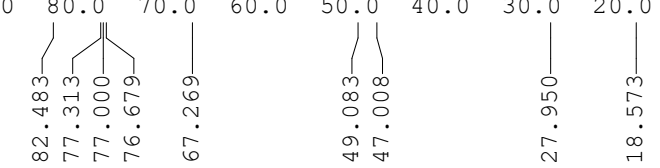




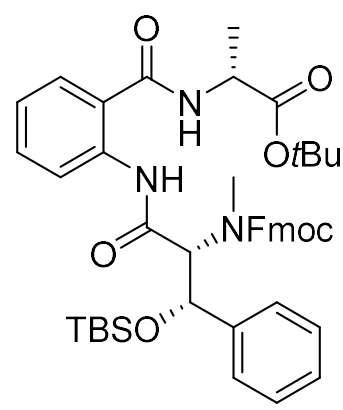

${ }^{1} \mathrm{H}$ NMR spectrum of $\mathbf{1 1 a}$

$11 \mathrm{a}$

${ }^{1} \mathrm{H}$ NMR

(400 $\mathrm{MHz}$ in $\mathrm{CDCl}_{3}$ )

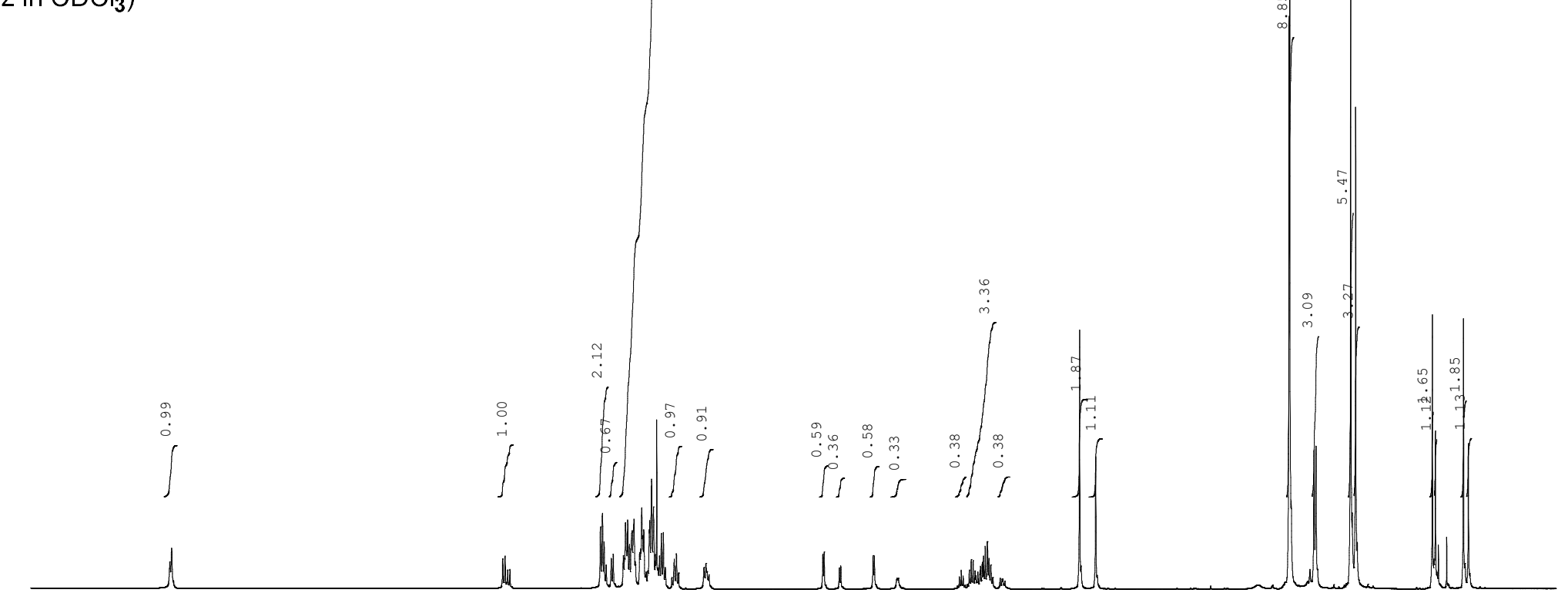

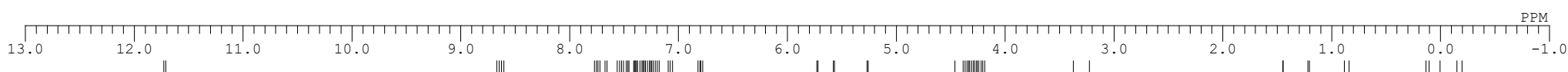

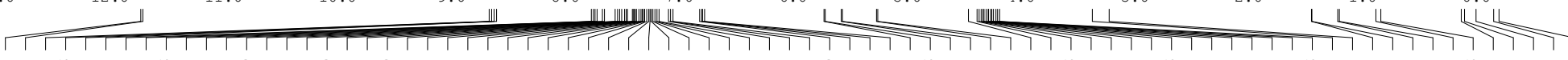

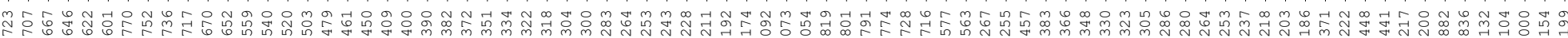

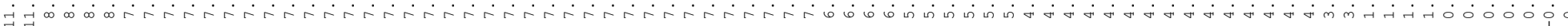




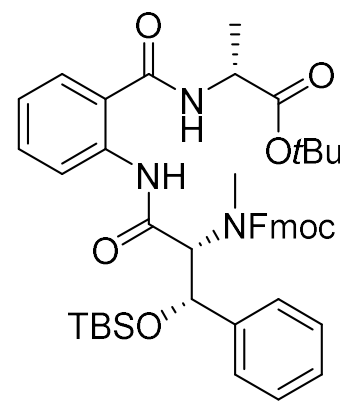

${ }^{13} \mathrm{C}$ NMR spectrum of $\mathbf{1 1 a}$

$11 a$

${ }^{13} \mathrm{C}$ NMR

$\left(100 \mathrm{MHz}\right.$ in $\mathrm{CDCl}_{3}$ )
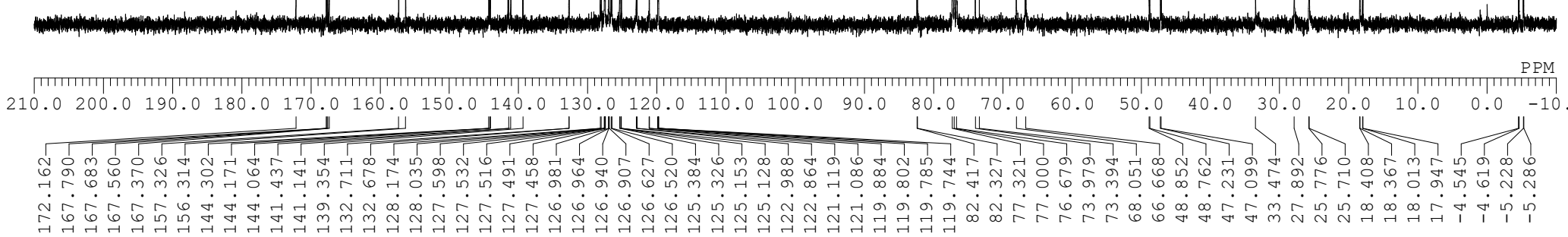


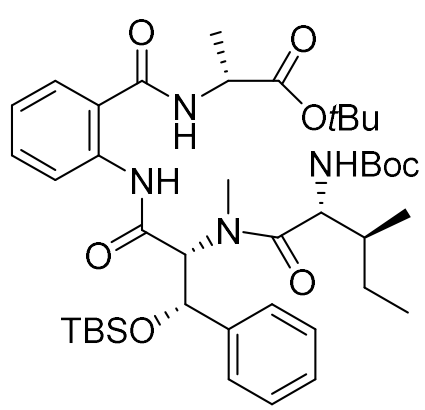

12

${ }^{1} \mathrm{H}$ NMR

$\left(400 \mathrm{MHz}\right.$ in $\left.\mathrm{CDCl}_{3}\right)$

${ }^{1} \mathrm{H}$ NMR spectrum of $\mathbf{1 2}$

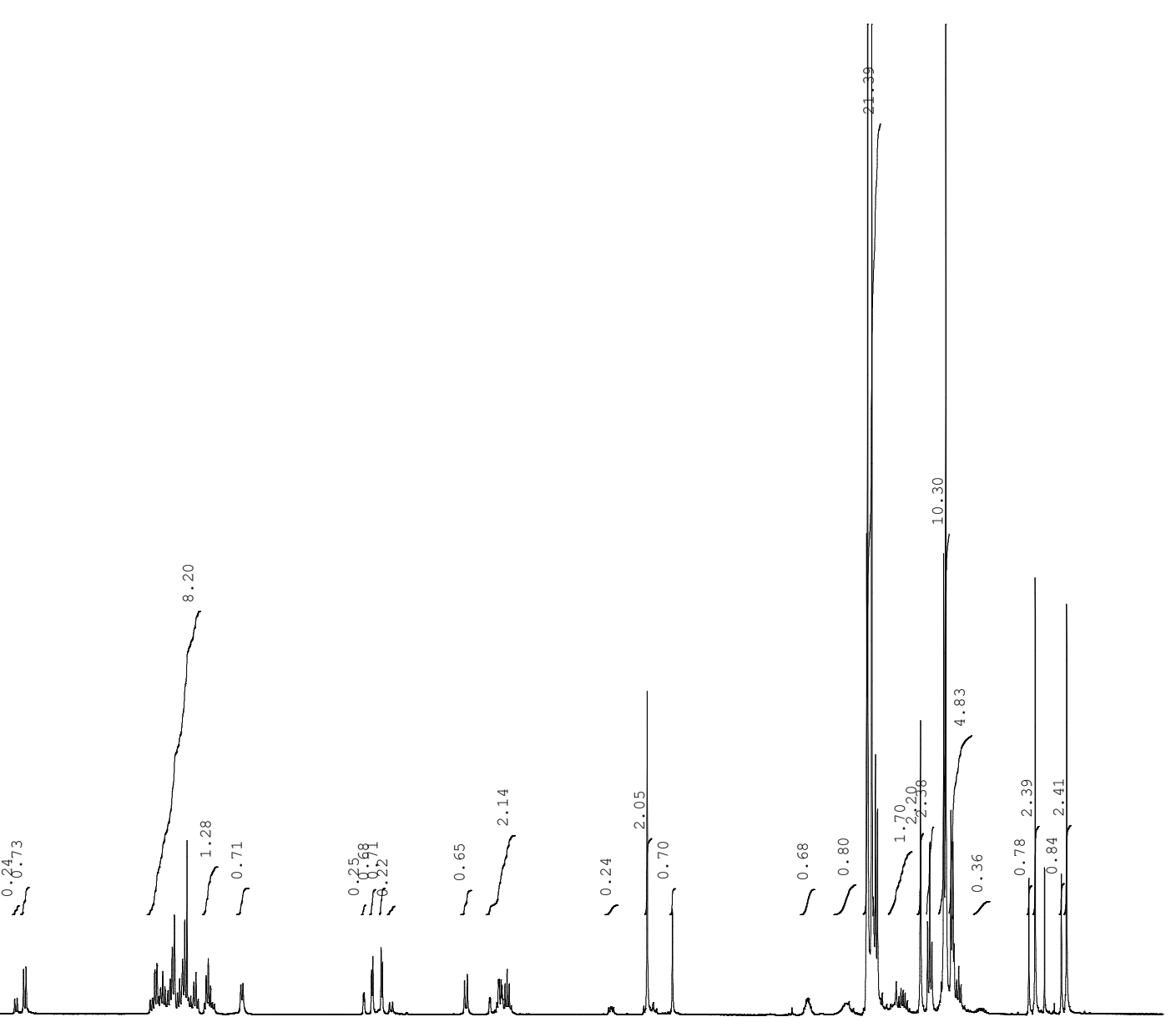

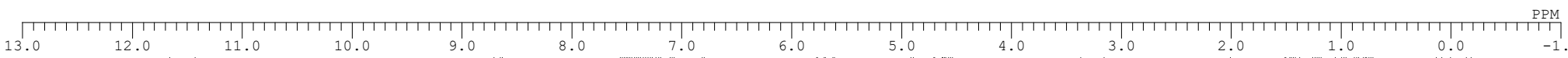

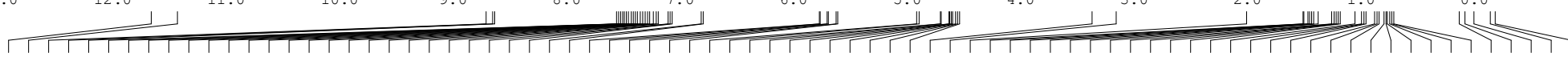

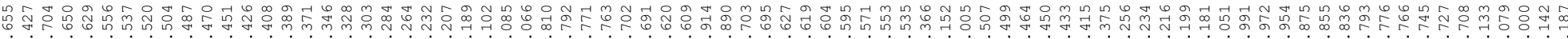
- 


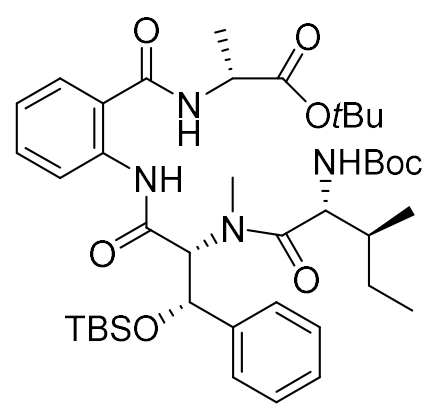

${ }^{13} \mathrm{C}$ NMR spectrum of $\mathbf{1 2}$

12

${ }^{13} \mathrm{C}$ NMR

$\left(100 \mathrm{MHz}\right.$ in $\left.\mathrm{CDCl}_{3}\right)$

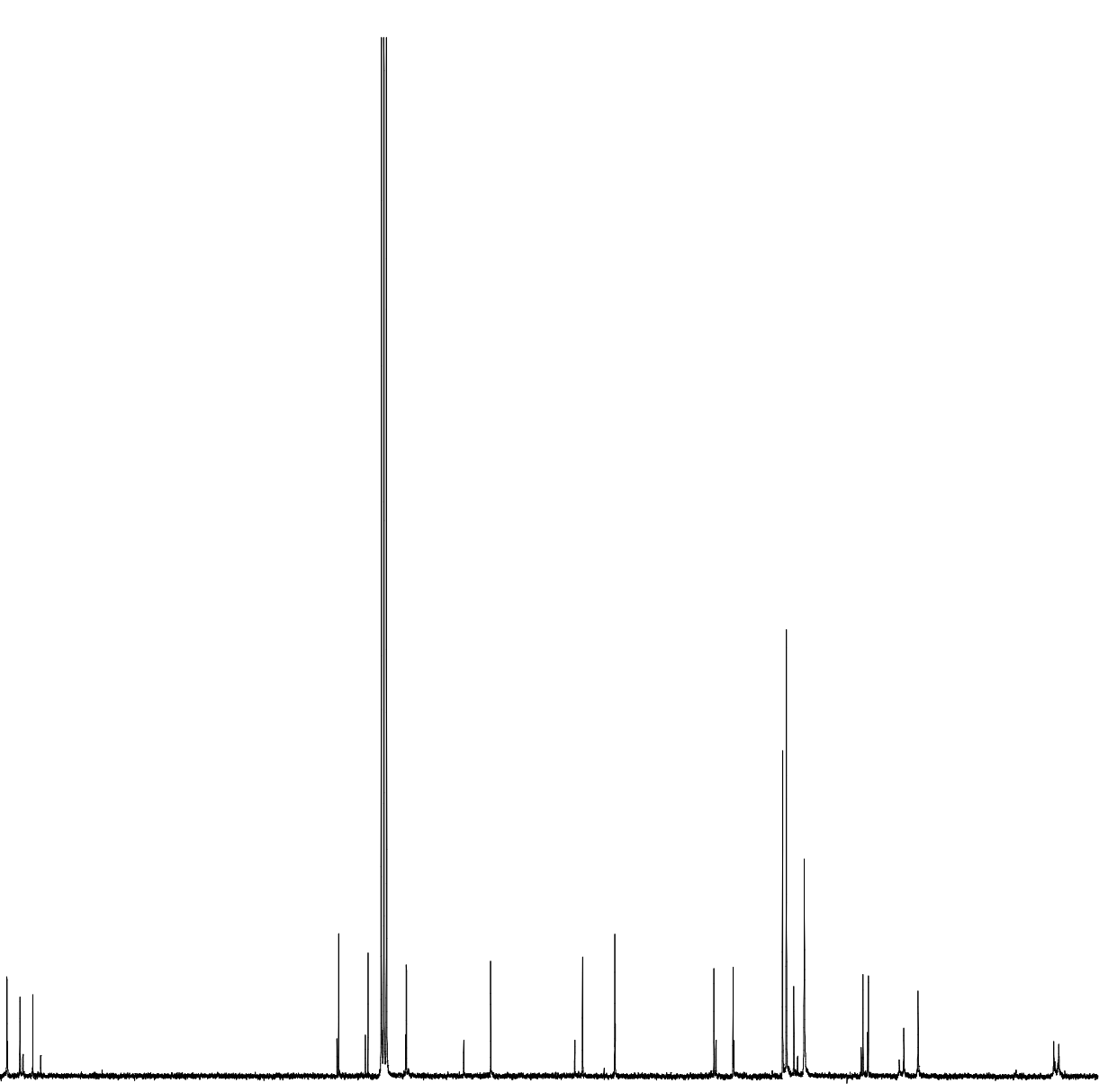

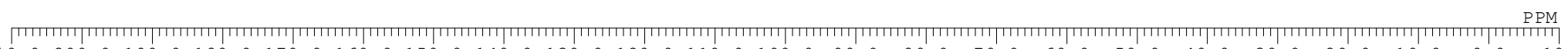

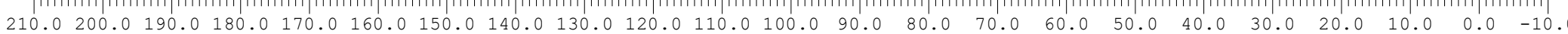

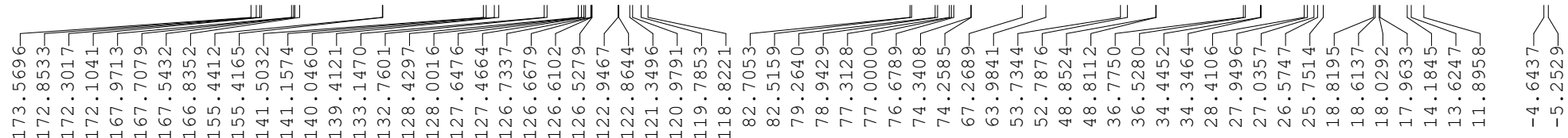




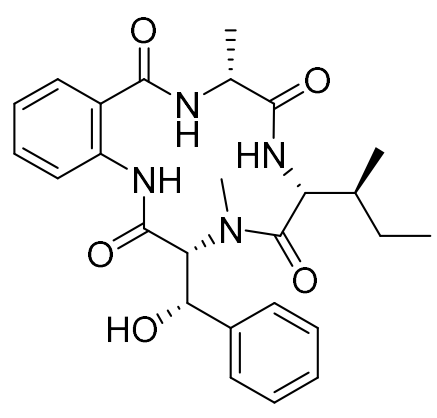

${ }^{1} \mathrm{H}$ NMR spectrum of $\mathbf{1 a}$

asperterrestide $A$

(1a, proposed)

${ }^{1} \mathrm{H}$ NMR

(600 $\mathrm{MHz}$ in $\mathrm{CDCl}_{3}$ )

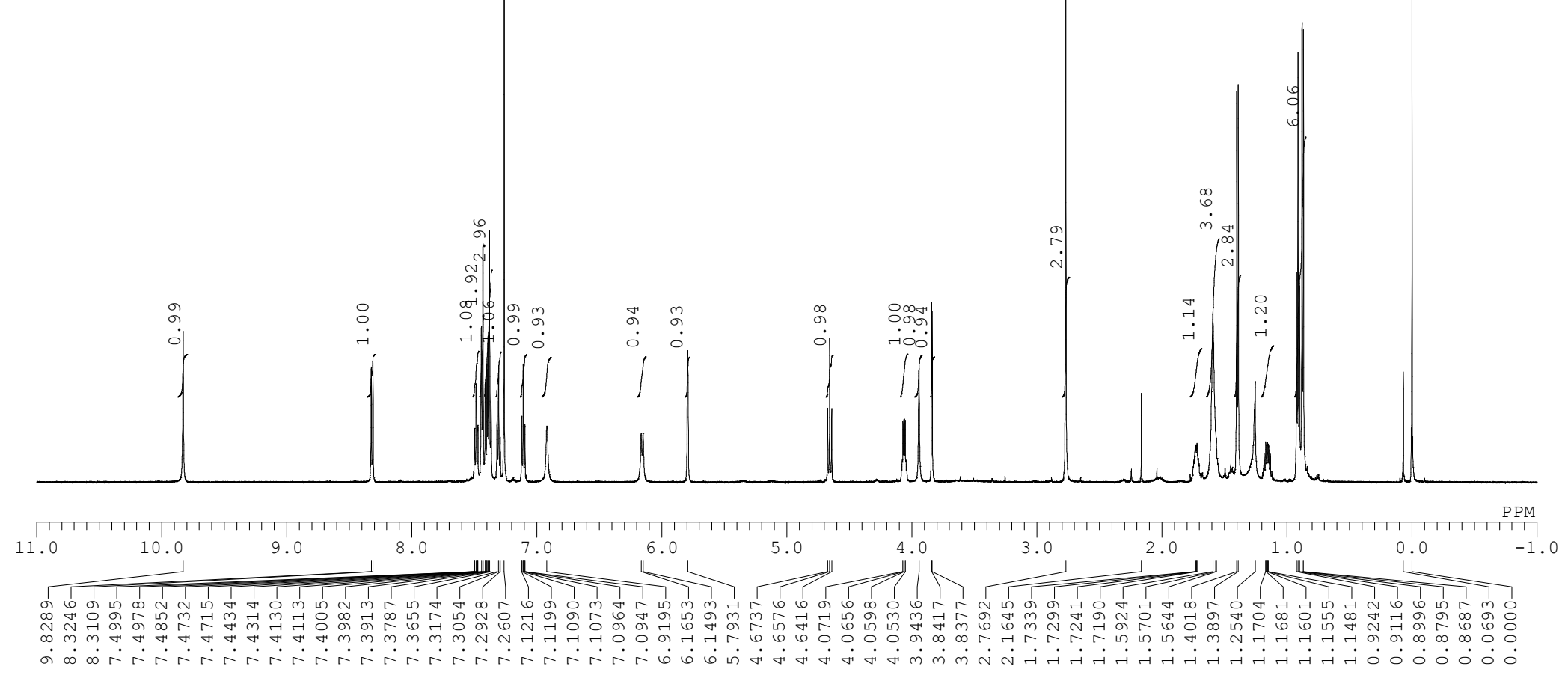




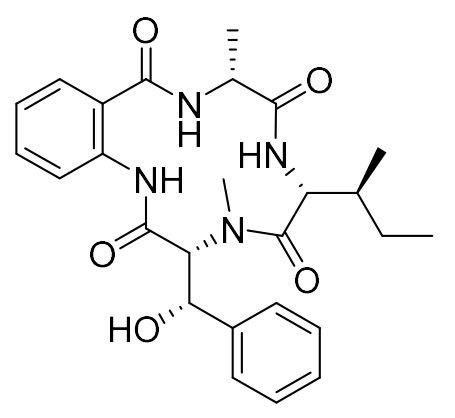

${ }^{13} \mathrm{C}$ NMR spectrum of $\mathbf{1 a}$

asperterrestide $A$

(1a, proposed)

${ }^{13} \mathrm{C}$ NMR

(150 $\mathrm{MHz}$ in $\mathrm{CDCl}_{3}$ )

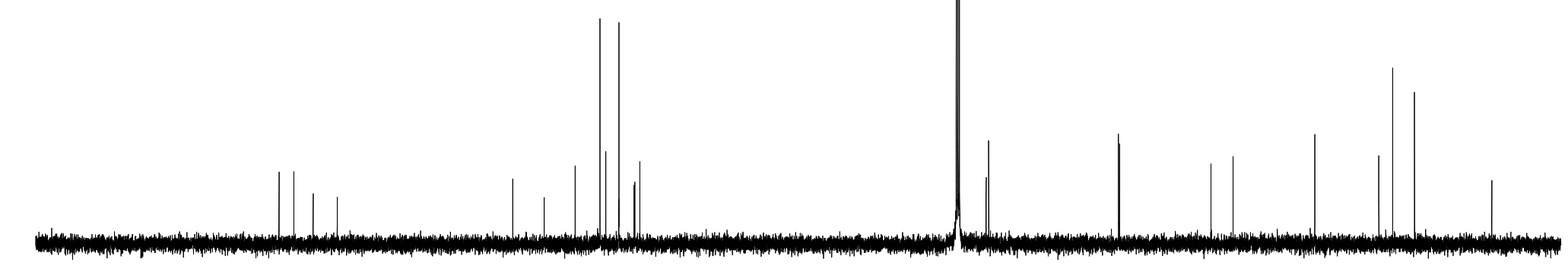

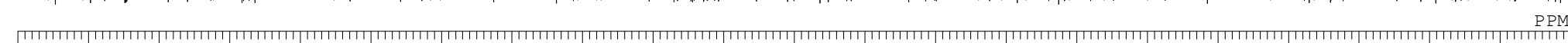

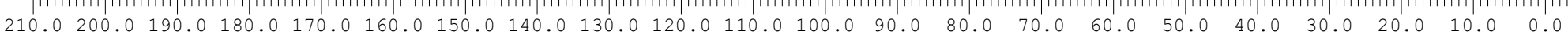
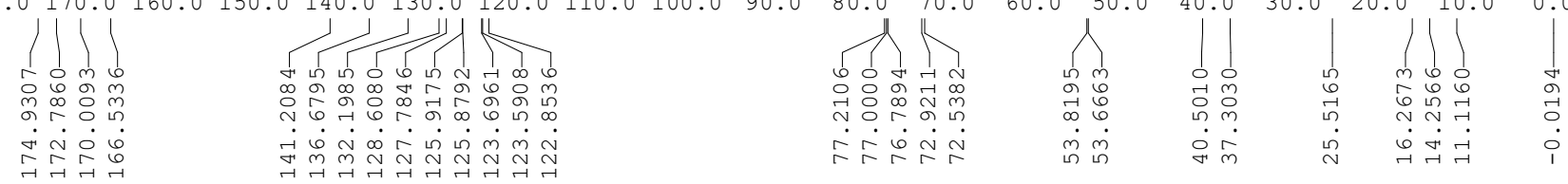
TBSO<smiles>CCC(C)NC(=O)OCc1ccccc1</smiles><smiles>CCC(=O)c1ccccc1</smiles>

ent-3

${ }^{1} \mathrm{H}$ NMR

(400 $\mathrm{MHz}$ in $\mathrm{CDCl}_{3}$ )

${ }^{1} \mathrm{H}$ NMR spectrum of ent-3

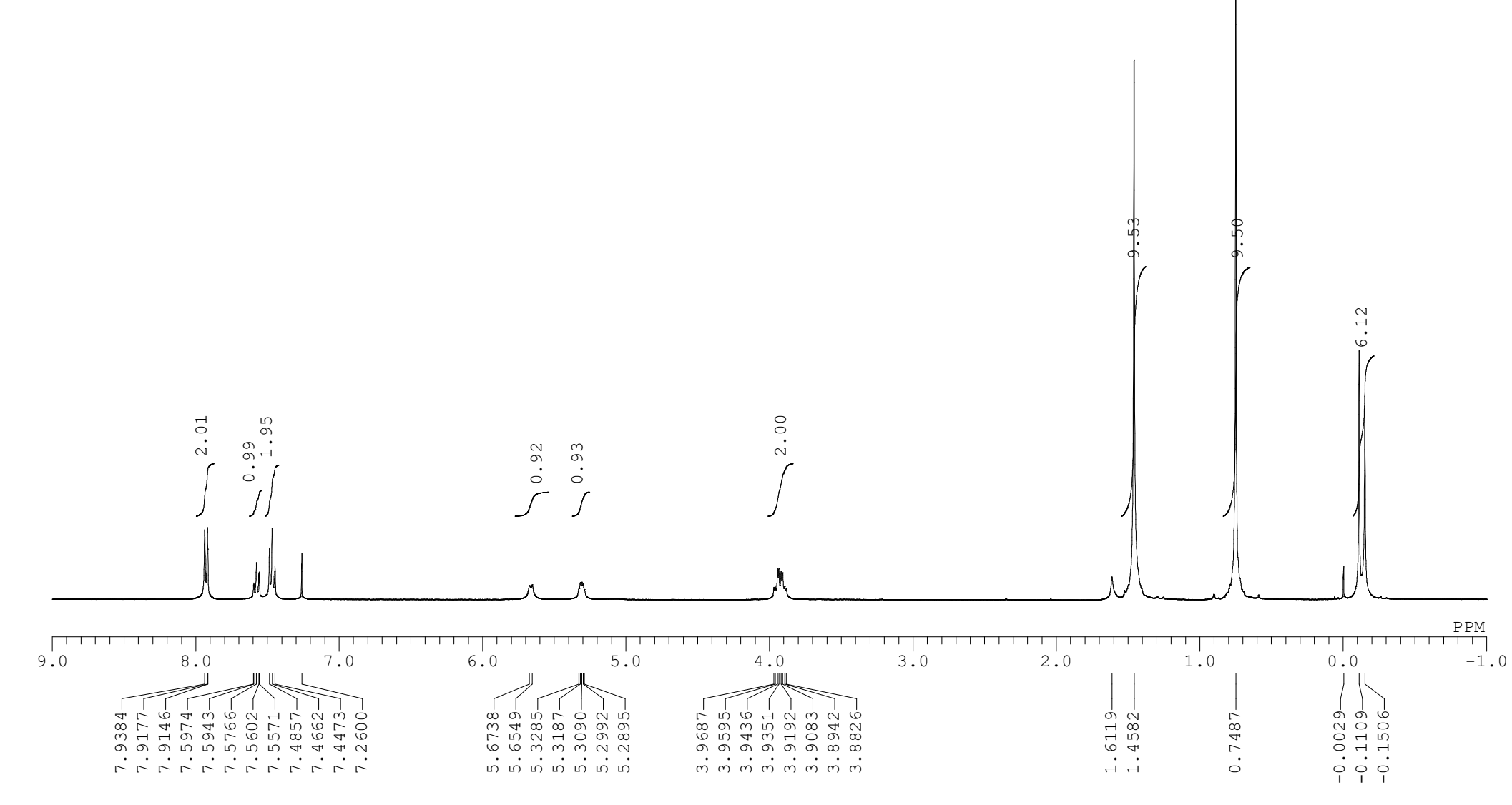


TBSO

${ }^{13} \mathrm{C}$ NMR spectrum of ent -3

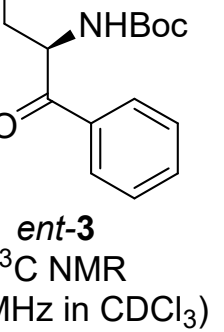

(100 $\mathrm{MHz}$ in $\mathrm{CDCl}_{3}$ )

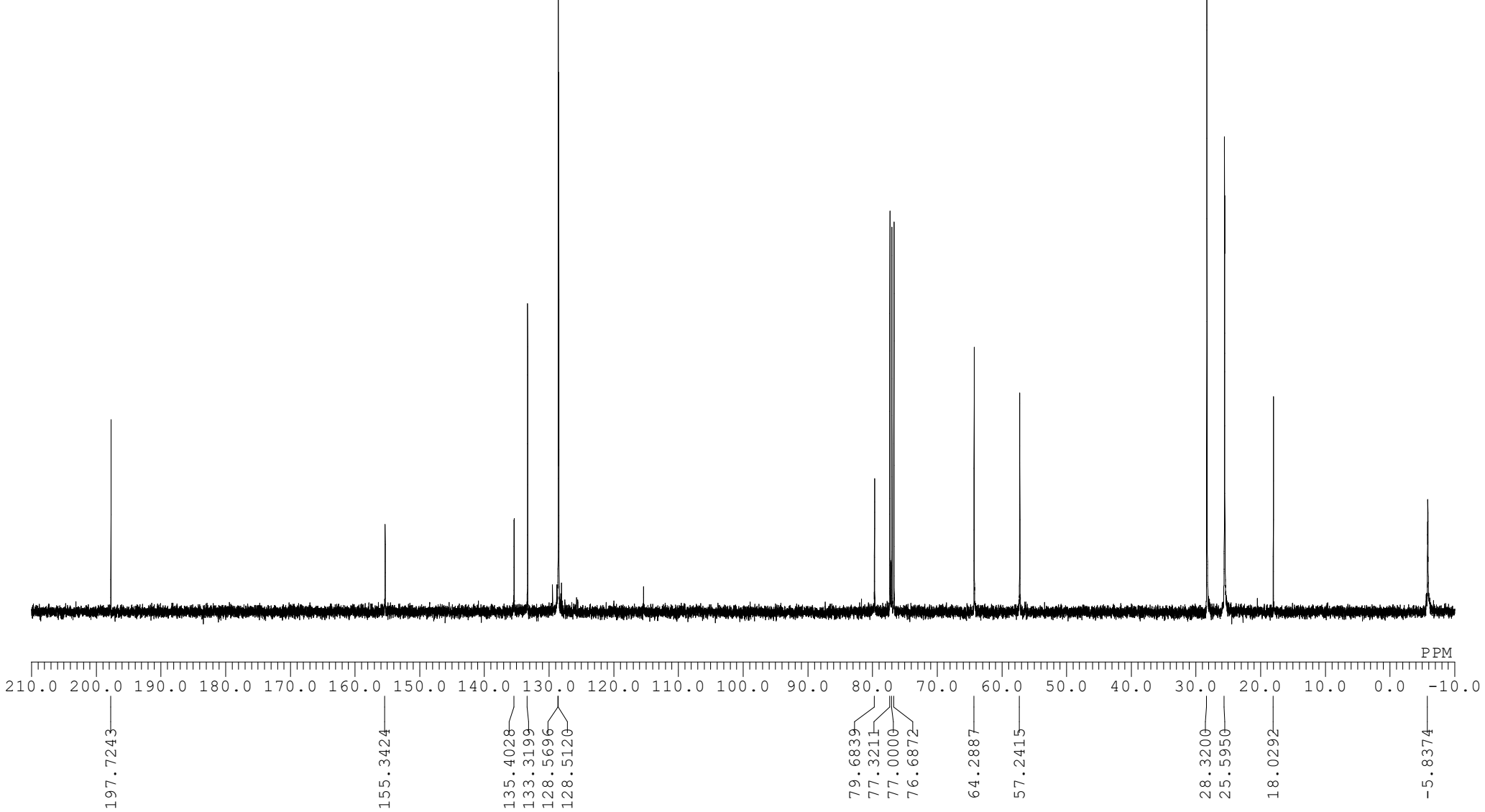


TBSO

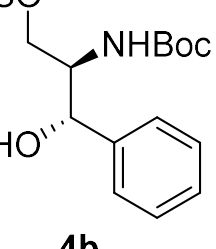

${ }^{1} \mathrm{H}$ NMR

(400 MHz in $\mathrm{CDCl}_{3}$ )

including diastreomer
${ }^{1} \mathrm{H}$ NMR spectrum of $\mathbf{4 b}$

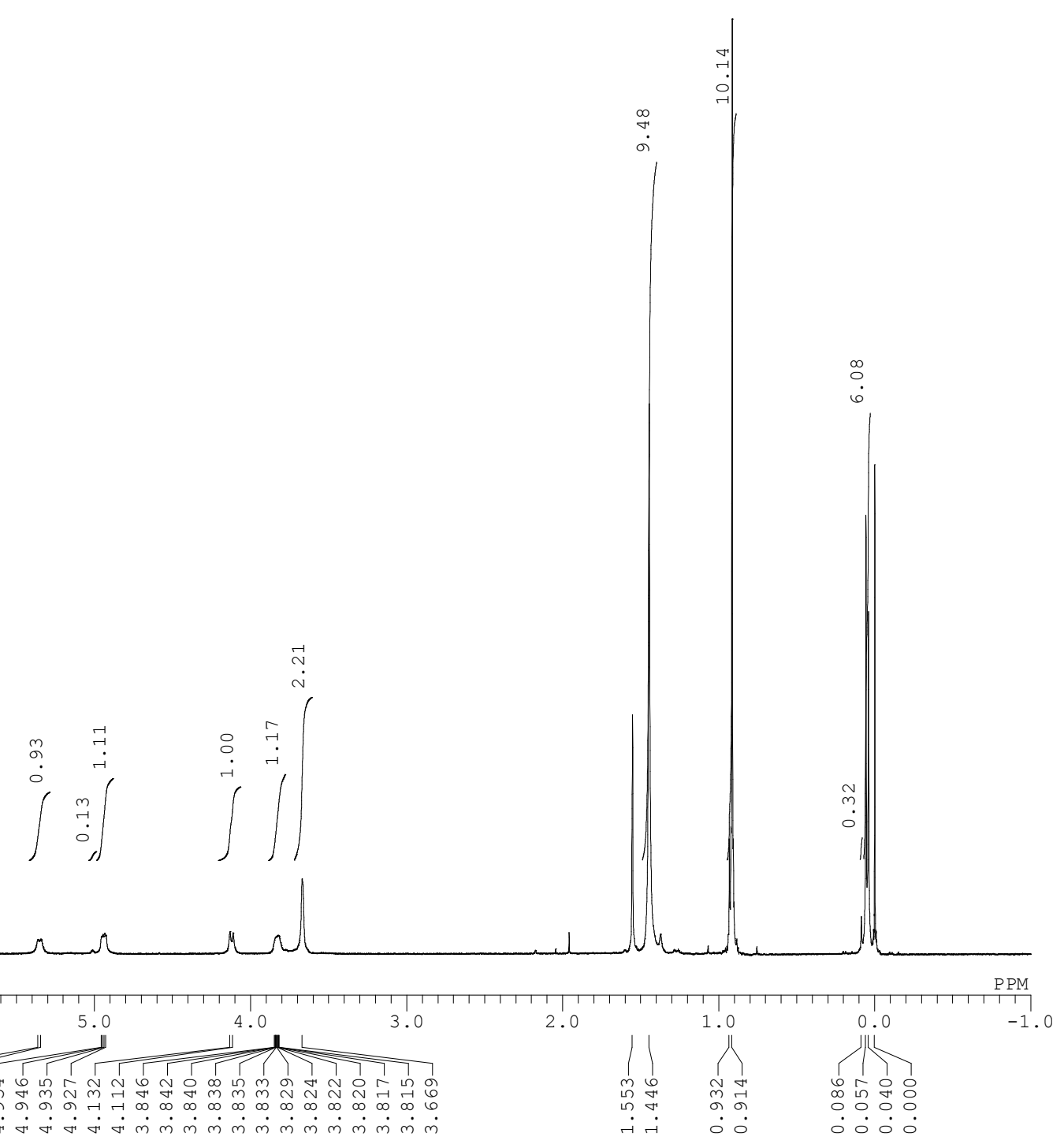

S29 


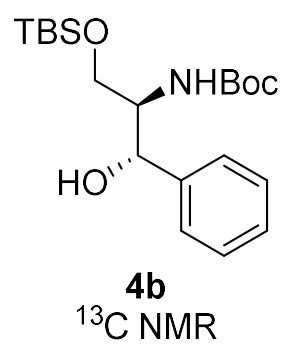

$\left(100 \mathrm{MHz}\right.$ in $\mathrm{CDCl}_{3}$ )

including diastreomer
${ }^{13} \mathrm{C}$ NMR spectrum of $\mathbf{4 b}$

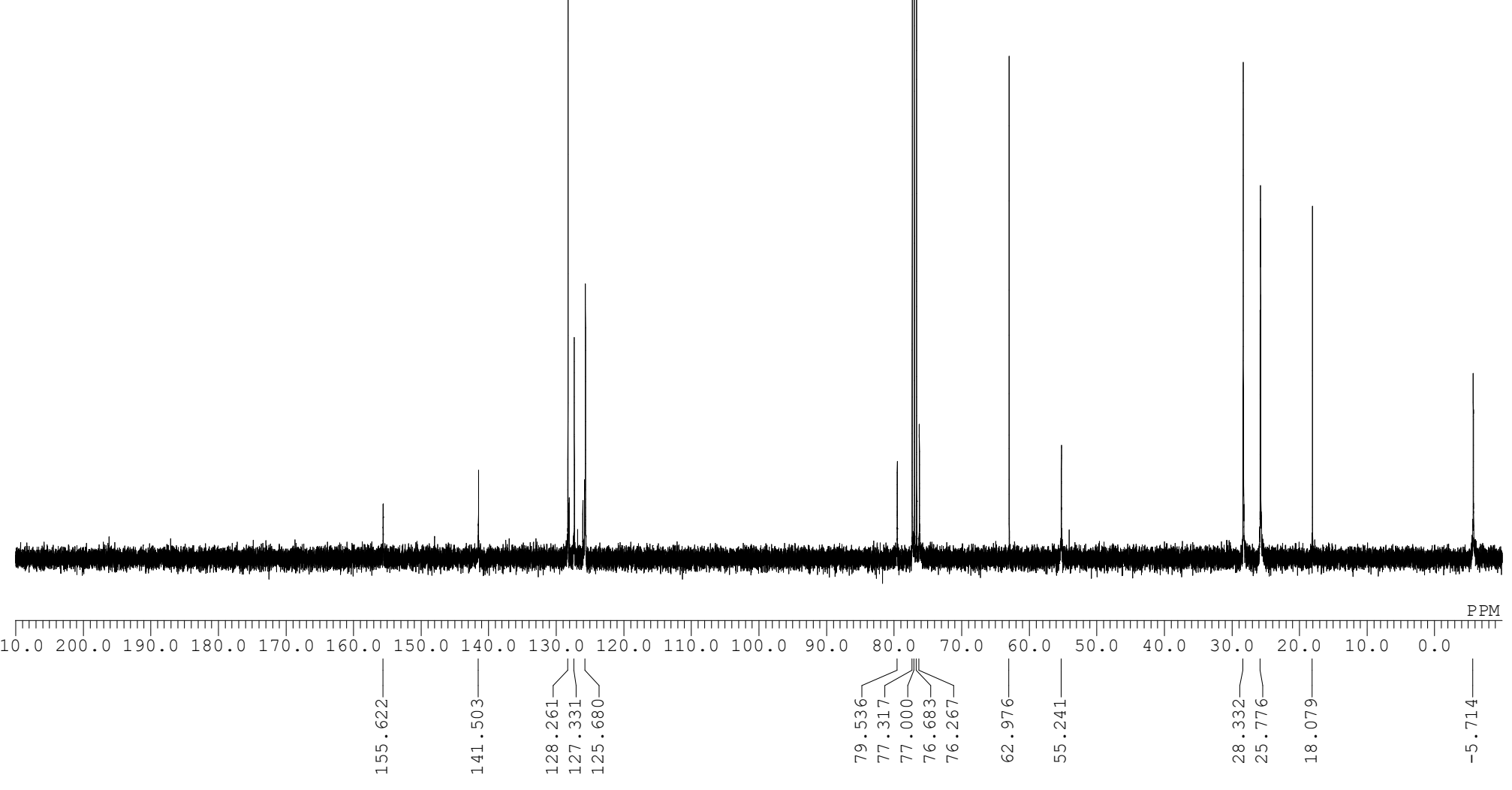




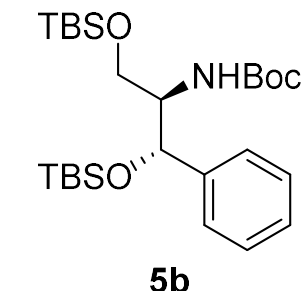

$5 b$

${ }^{1} \mathrm{H}$ NMR

(400 $\mathrm{MHz}$ in $\mathrm{CDCl}_{3}$ )

including diastreomer

${ }^{1} \mathrm{H}$ NMR spectrum of $\mathbf{5 b}$

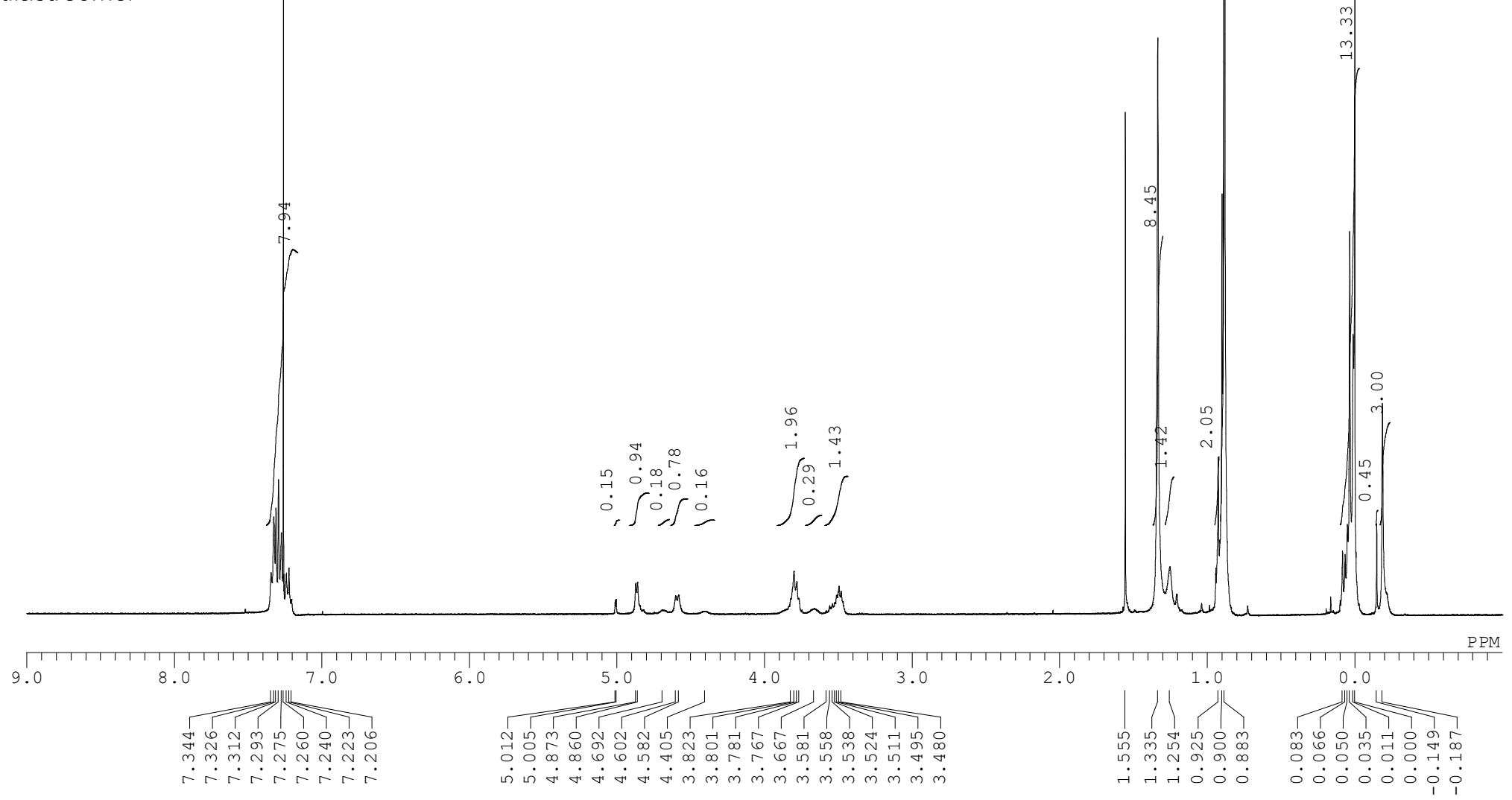




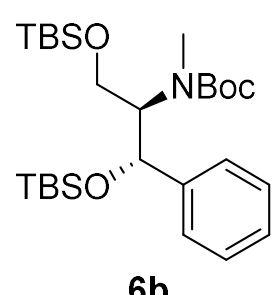

$6 \mathrm{~b}$

${ }^{1} \mathrm{H}$ NMR

(400 $\mathrm{MHz}$ in $\mathrm{CDCl}_{3}$ )

including diastereomer

${ }^{1} \mathrm{H}$ NMR spectrum of $\mathbf{6 b}$

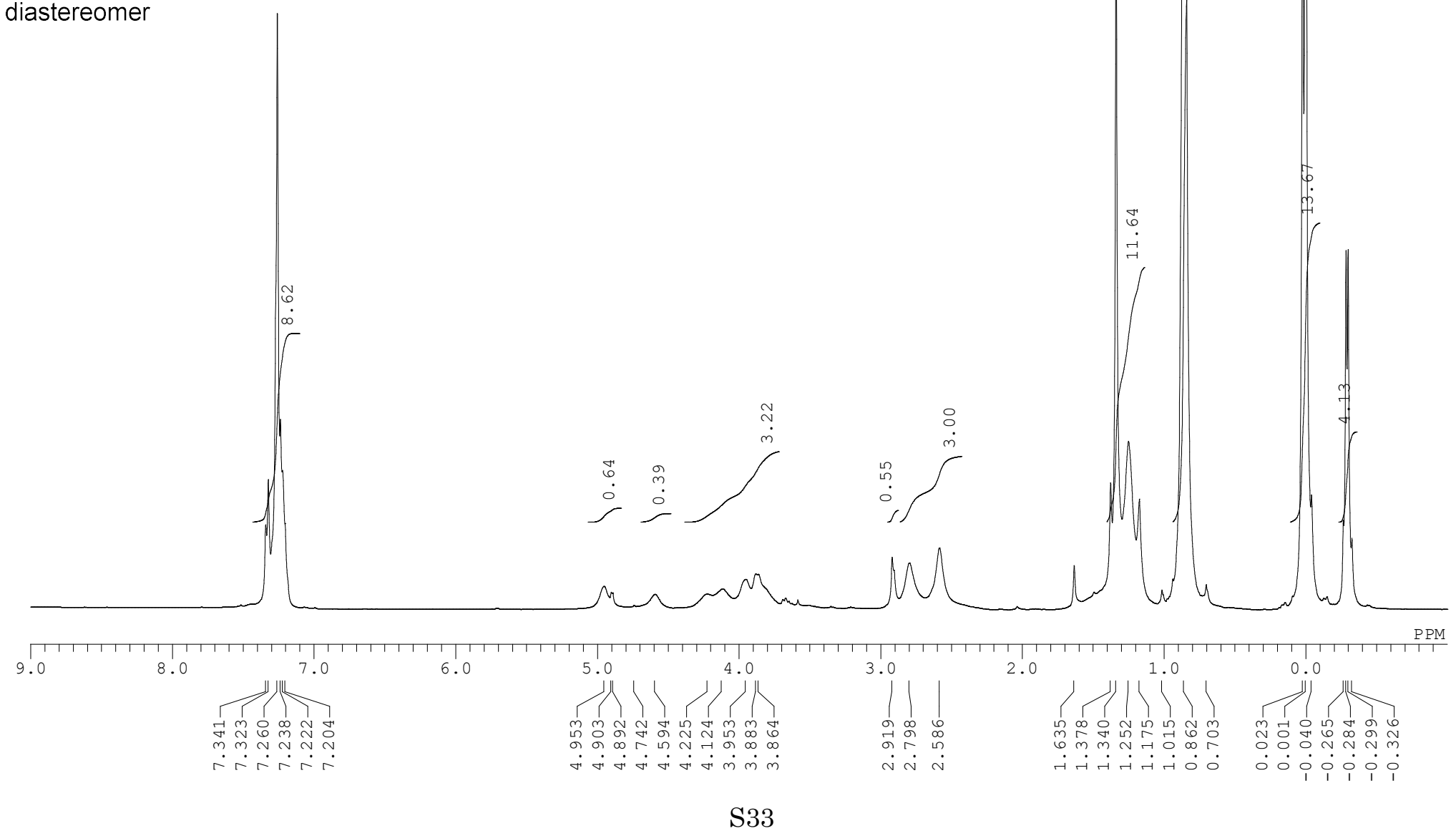




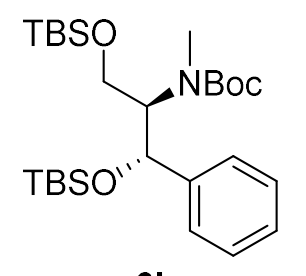

${ }^{13} \mathrm{C}$ NMR spectrum of $\mathbf{6 b}$

$6 b$

${ }^{13} \mathrm{C}$ NMR

$\left(100 \mathrm{MHz}\right.$ in $\mathrm{CDCl}_{3}$ )

including diastereomer

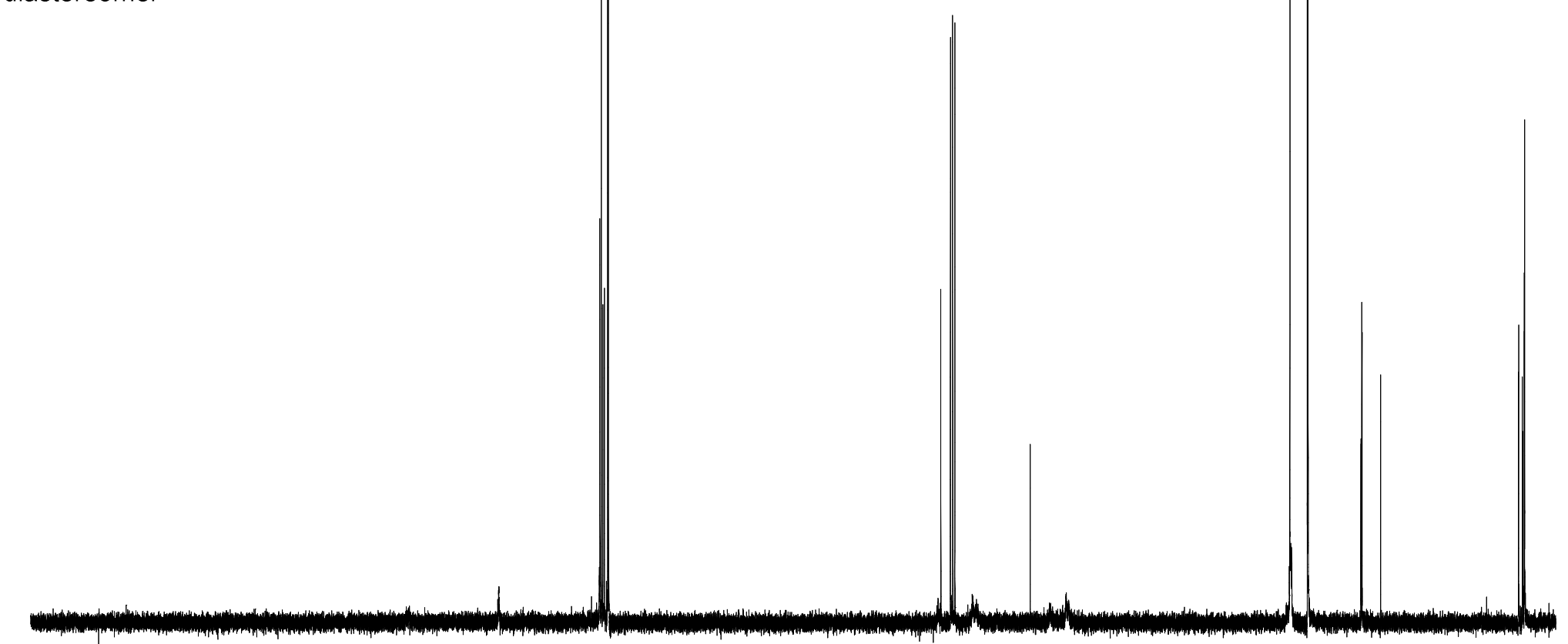

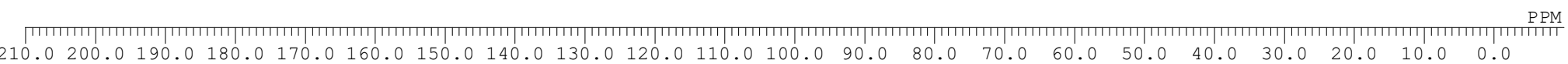
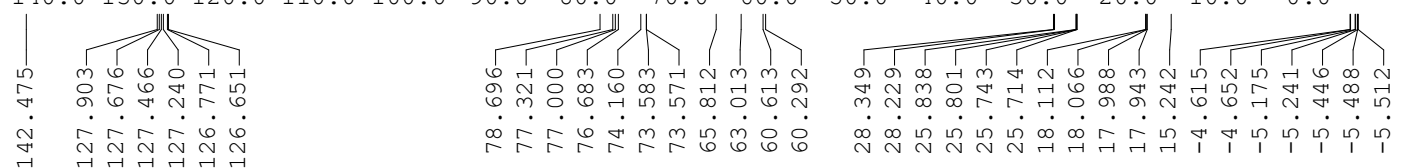


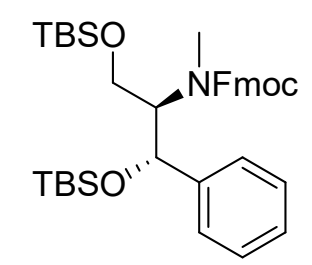

${ }^{1} \mathrm{H}$ NMR spectrum of $\mathbf{7 b}$

7b

${ }^{1} \mathrm{H}$ NMR

(400 MHz in $\mathrm{CDCl}_{3}$ )

including diastereomer

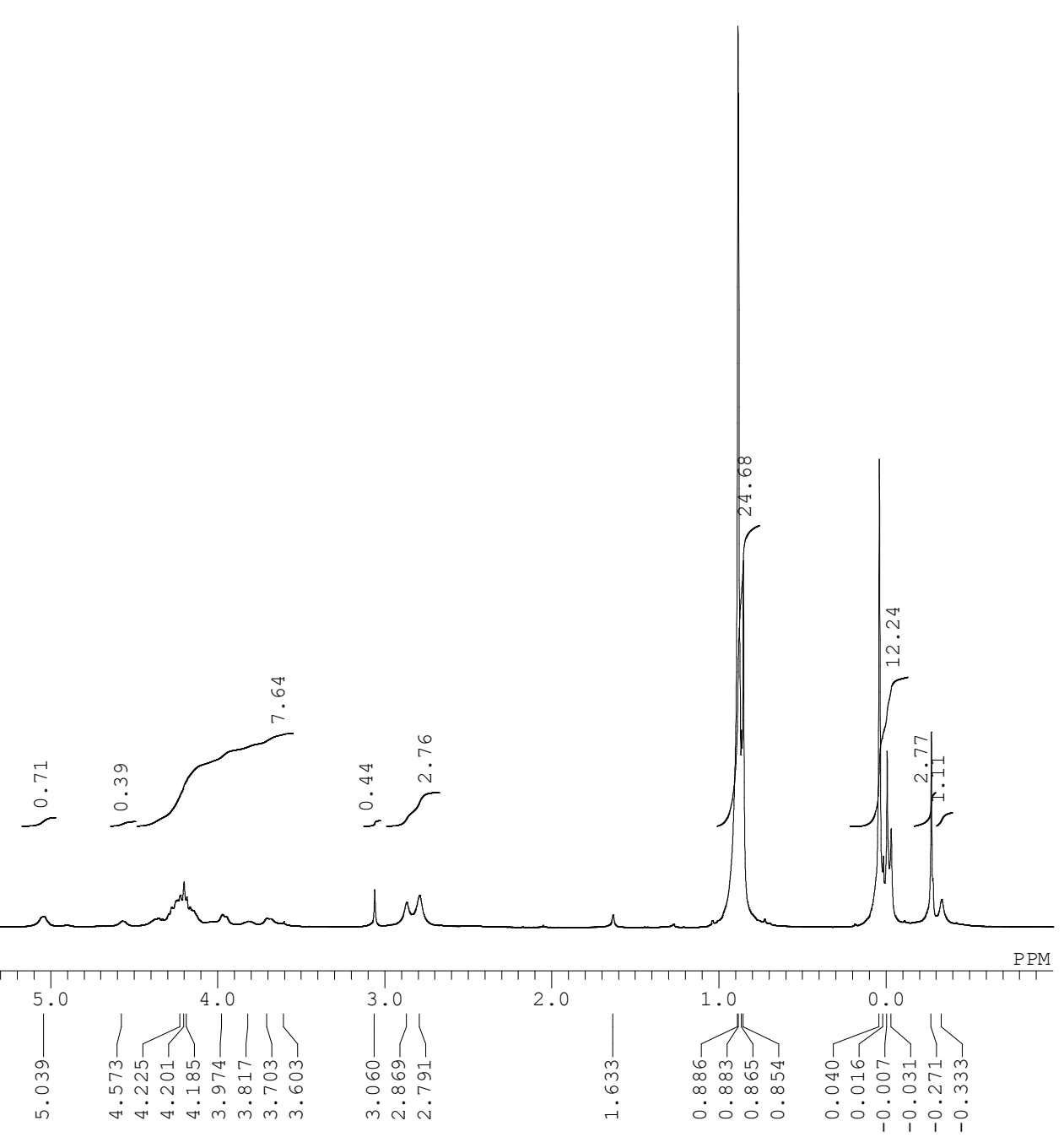




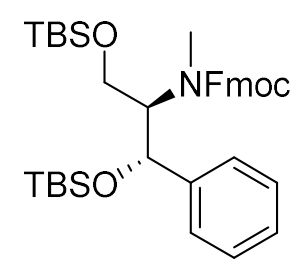

${ }^{13} \mathrm{C}$ NMR spectrum of $7 \mathbf{b}$

$7 b$

${ }^{13} \mathrm{C}$ NMR

$\left(100 \mathrm{MHz}\right.$ in $\left.\mathrm{CDCl}_{3}\right)$

including diastereomer

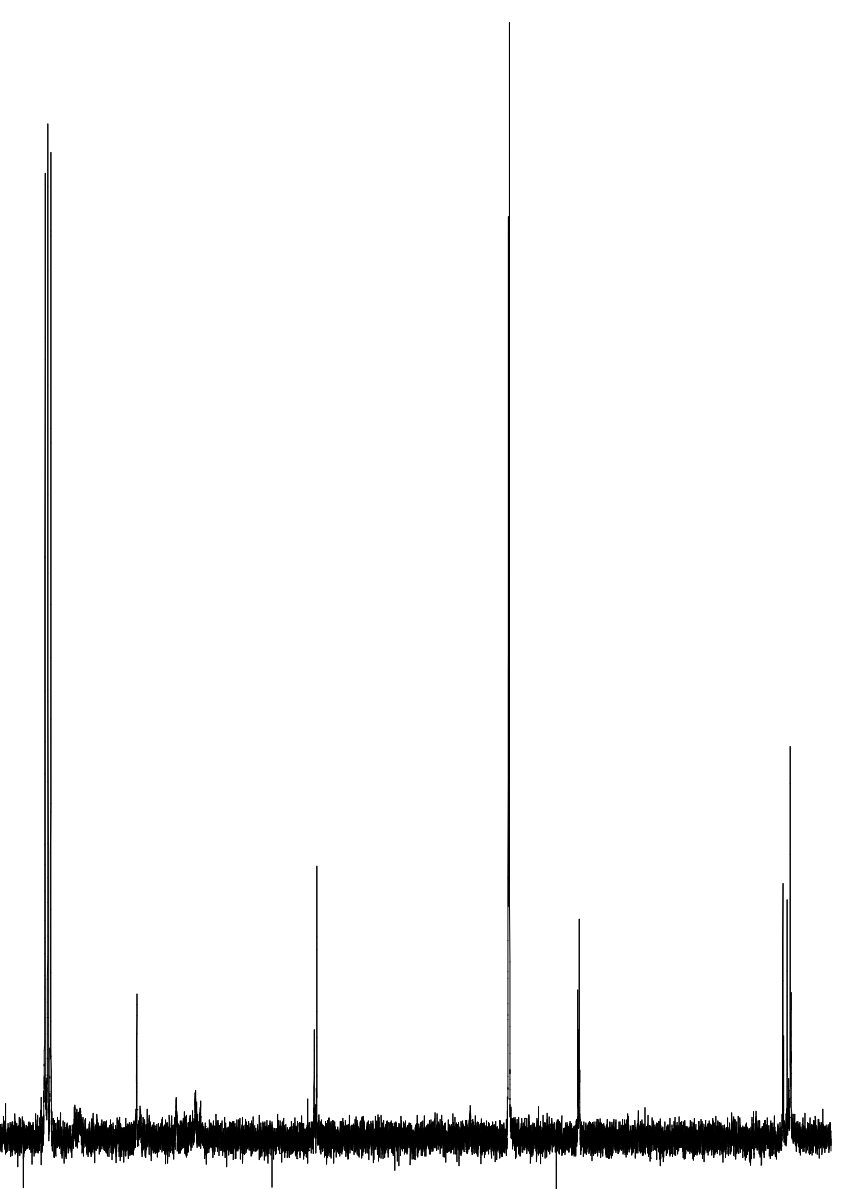

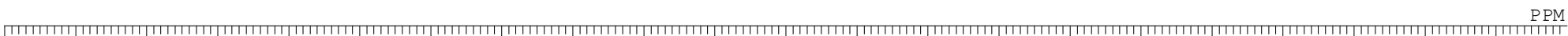
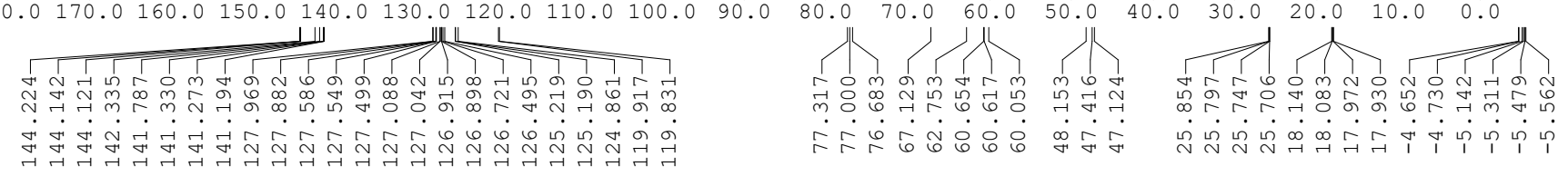


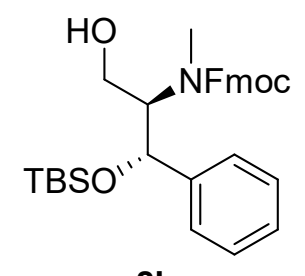

${ }^{1} \mathrm{H}$ NMR spectrum of $\mathbf{8 b}$

ab

${ }^{1} \mathrm{H}$ NMR

(400 $\mathrm{MHz}$ in $\mathrm{CDCl}_{3}$ )

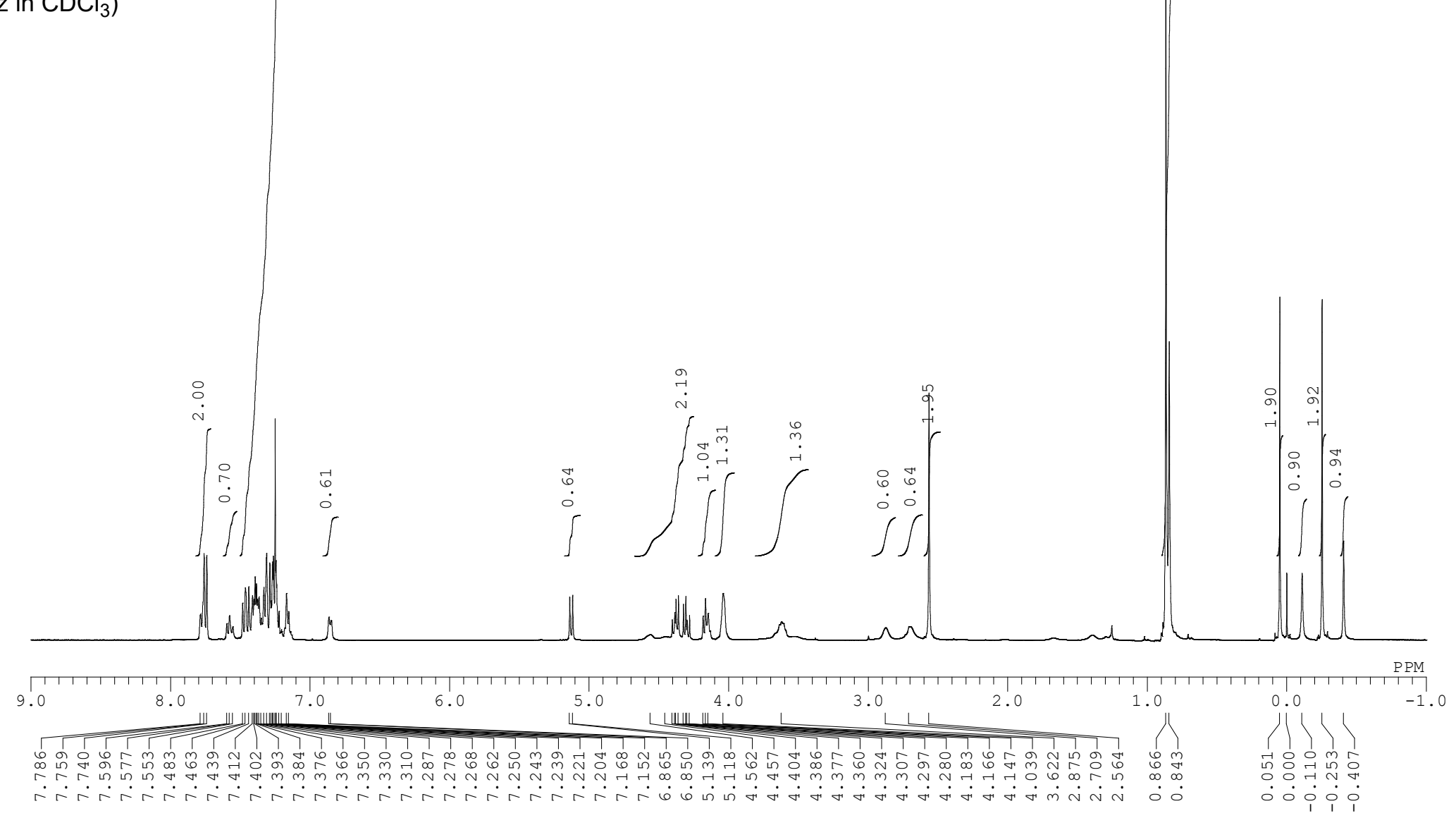




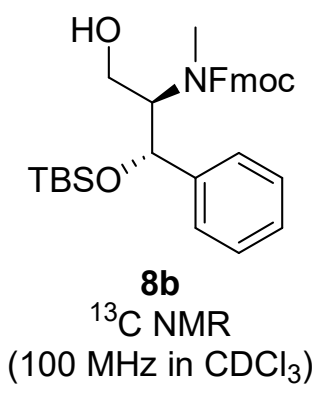

${ }^{13} \mathrm{C}$ NMR spectrum of $\mathbf{8 b}$

$\left(100 \mathrm{MHz}\right.$ in $\left.\mathrm{CDCl}_{3}\right)$
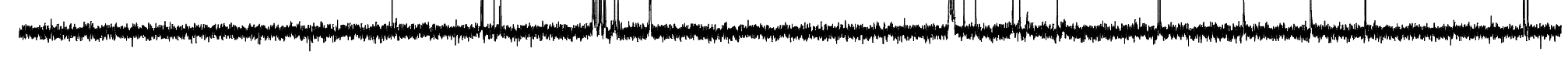

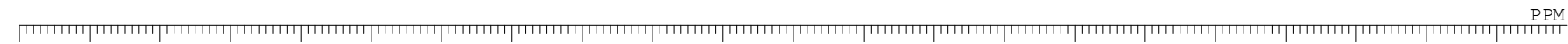

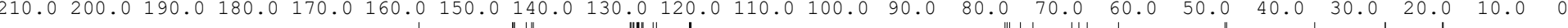
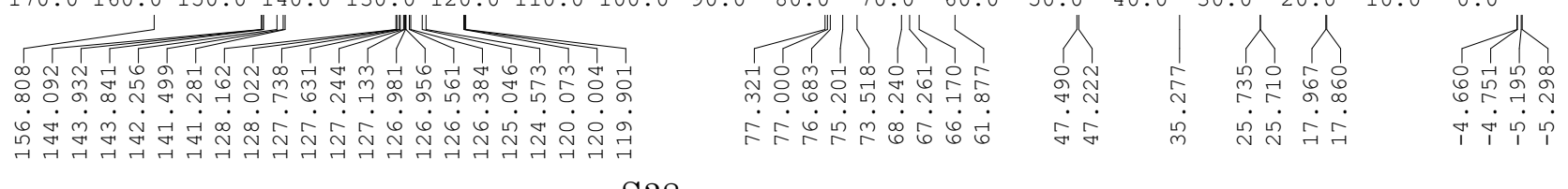


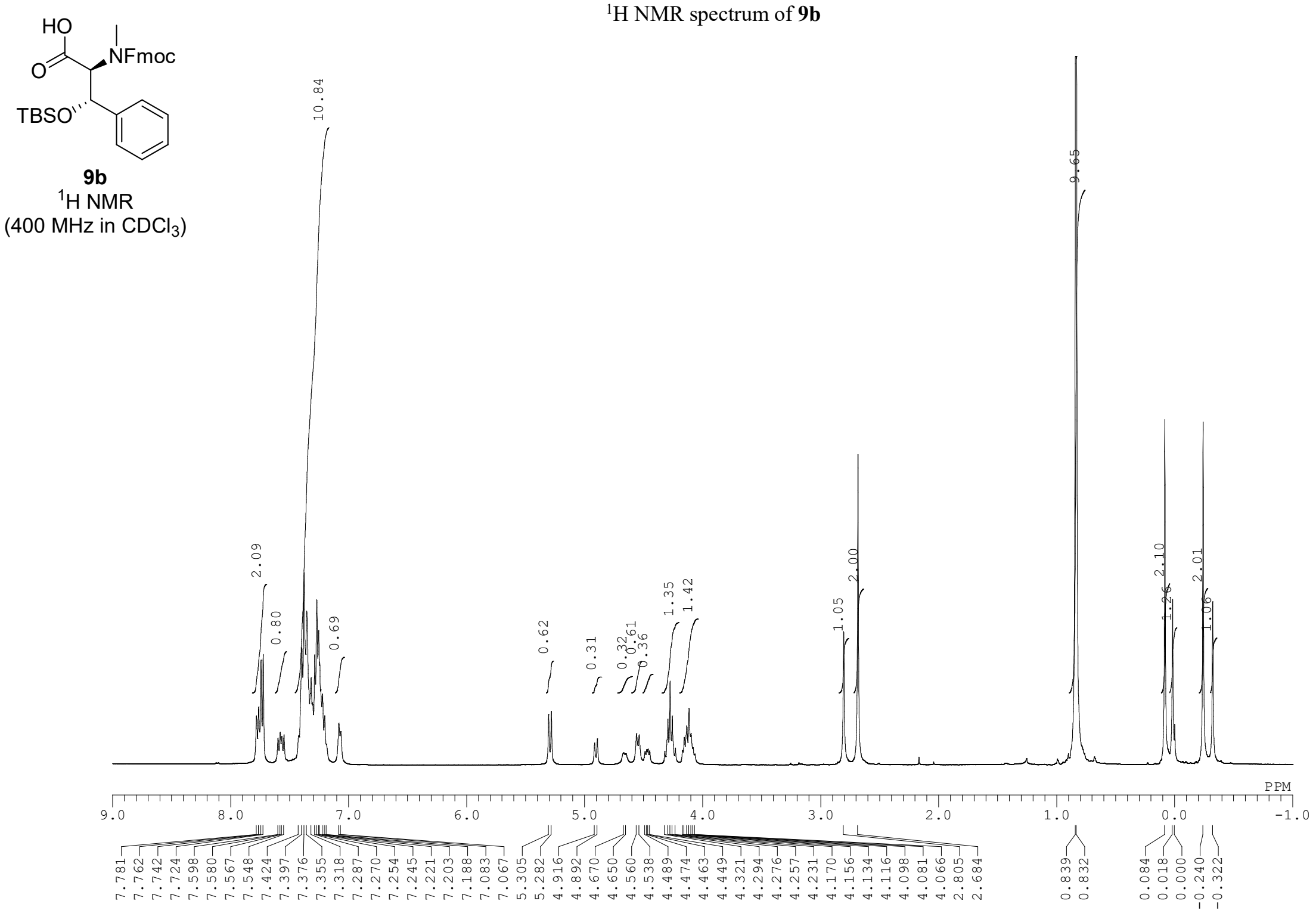




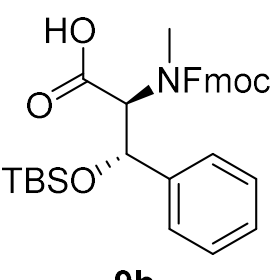

$9 b$

${ }^{13} \mathrm{C} N M R$

$\left(100 \mathrm{MHz}\right.$ in $\left.\mathrm{CDCl}_{3}\right)$

${ }^{13} \mathrm{C}$ NMR spectrum of $\mathbf{9 b}$

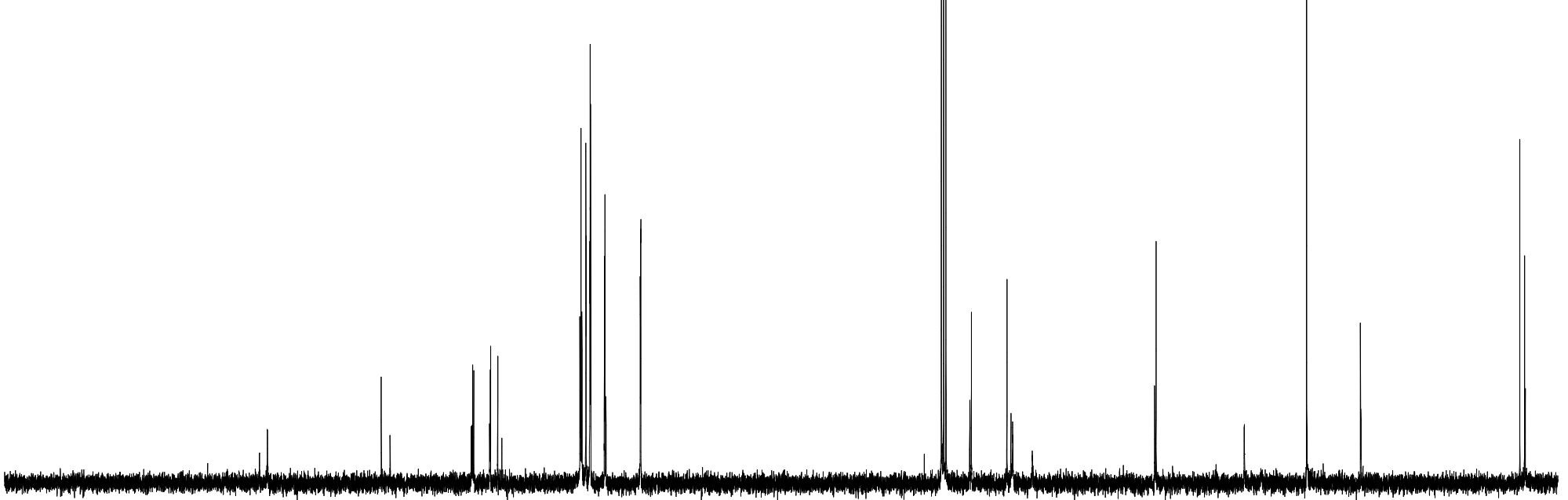

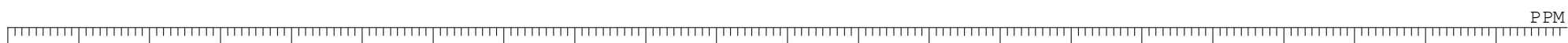

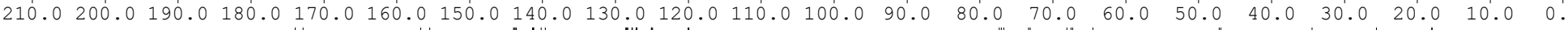
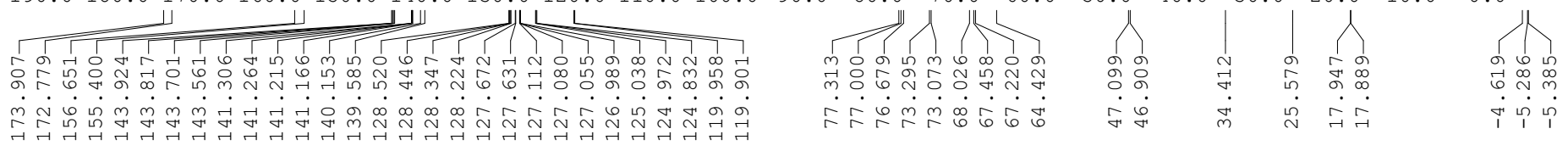


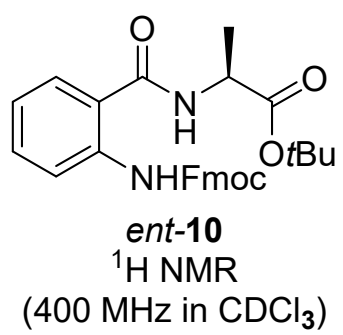

${ }^{1} \mathrm{H}$ NMR spectrum of ent-10

$\left(400 \mathrm{MHz}\right.$ in $\mathrm{CDCl}_{3}$ )

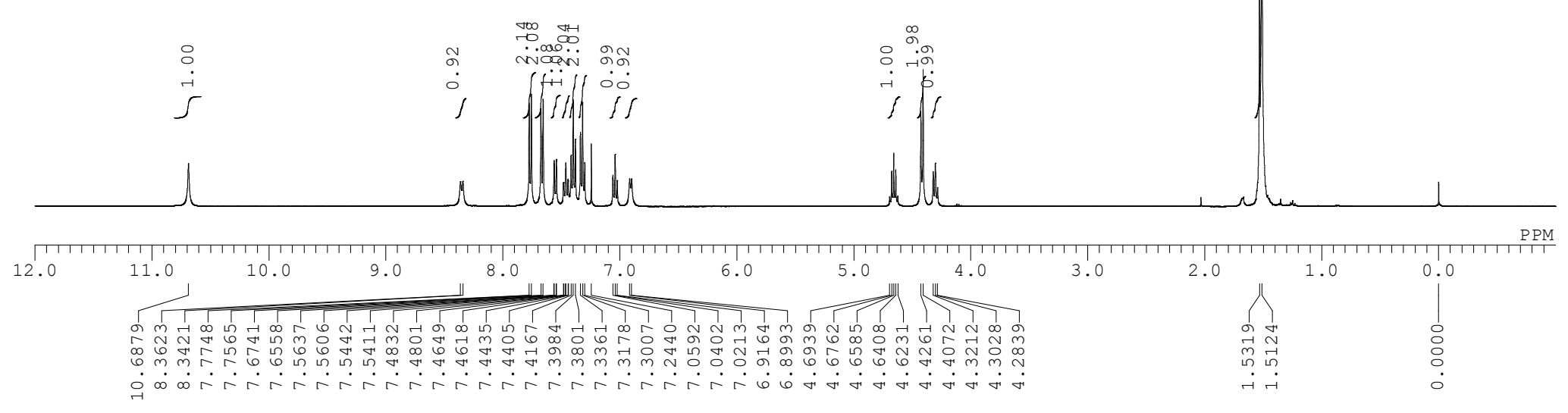




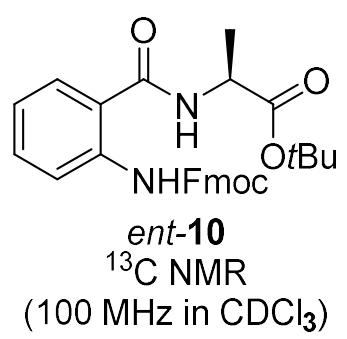

${ }^{13} \mathrm{C}$ NMR spectrum of ent-10

$\left(100 \mathrm{MHz}\right.$ in $\left.\mathrm{CDCl}_{3}\right)$

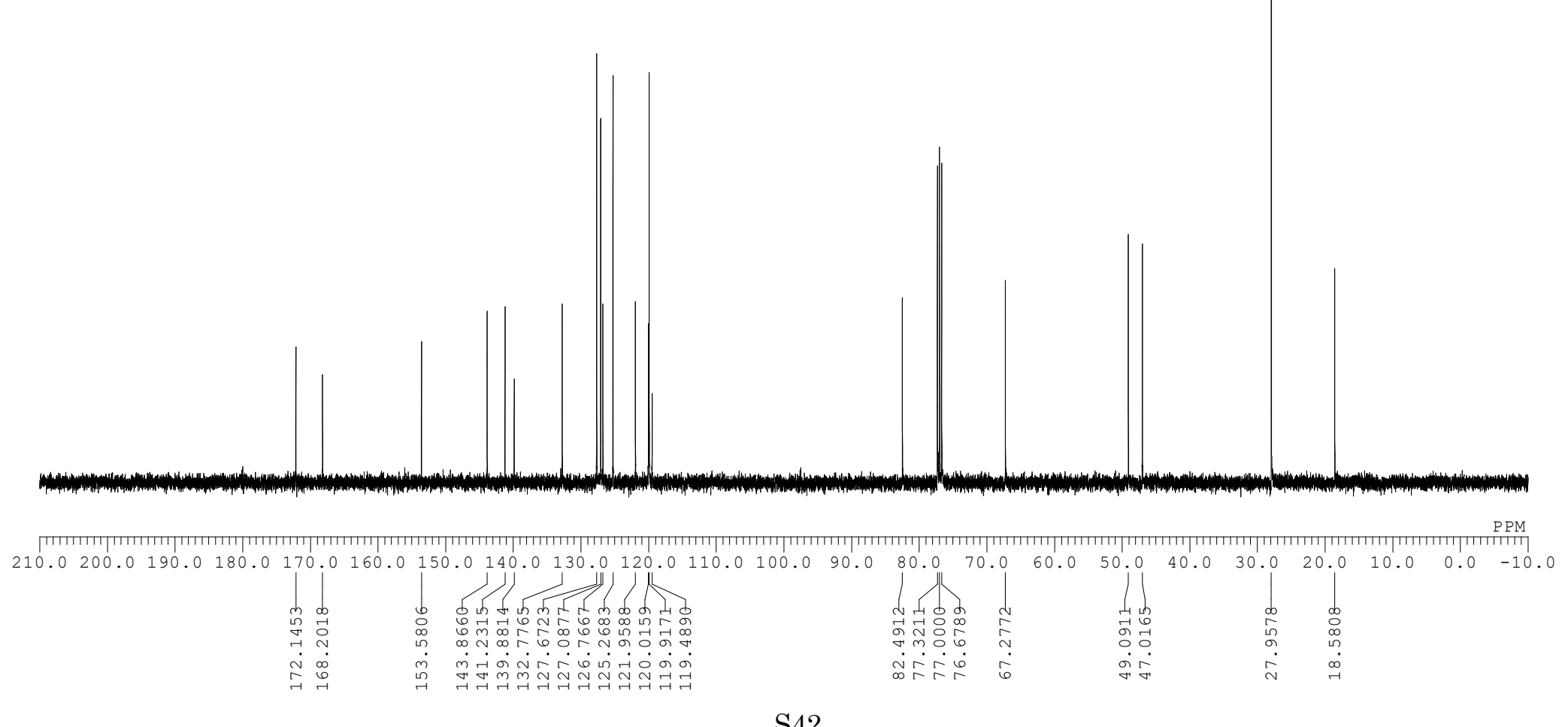




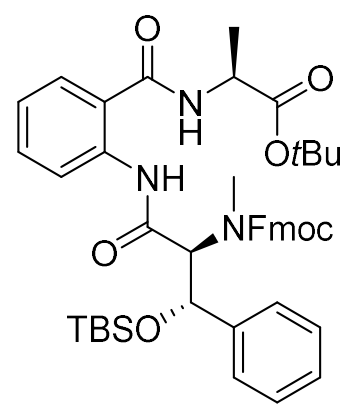

11b

${ }^{1} \mathrm{H}$ NMR

(400 $\mathrm{MHz}$ in $\mathrm{CDCl}_{3}$ )

${ }^{1} \mathrm{H}$ NMR spectrum of $\mathbf{1 1 b}$

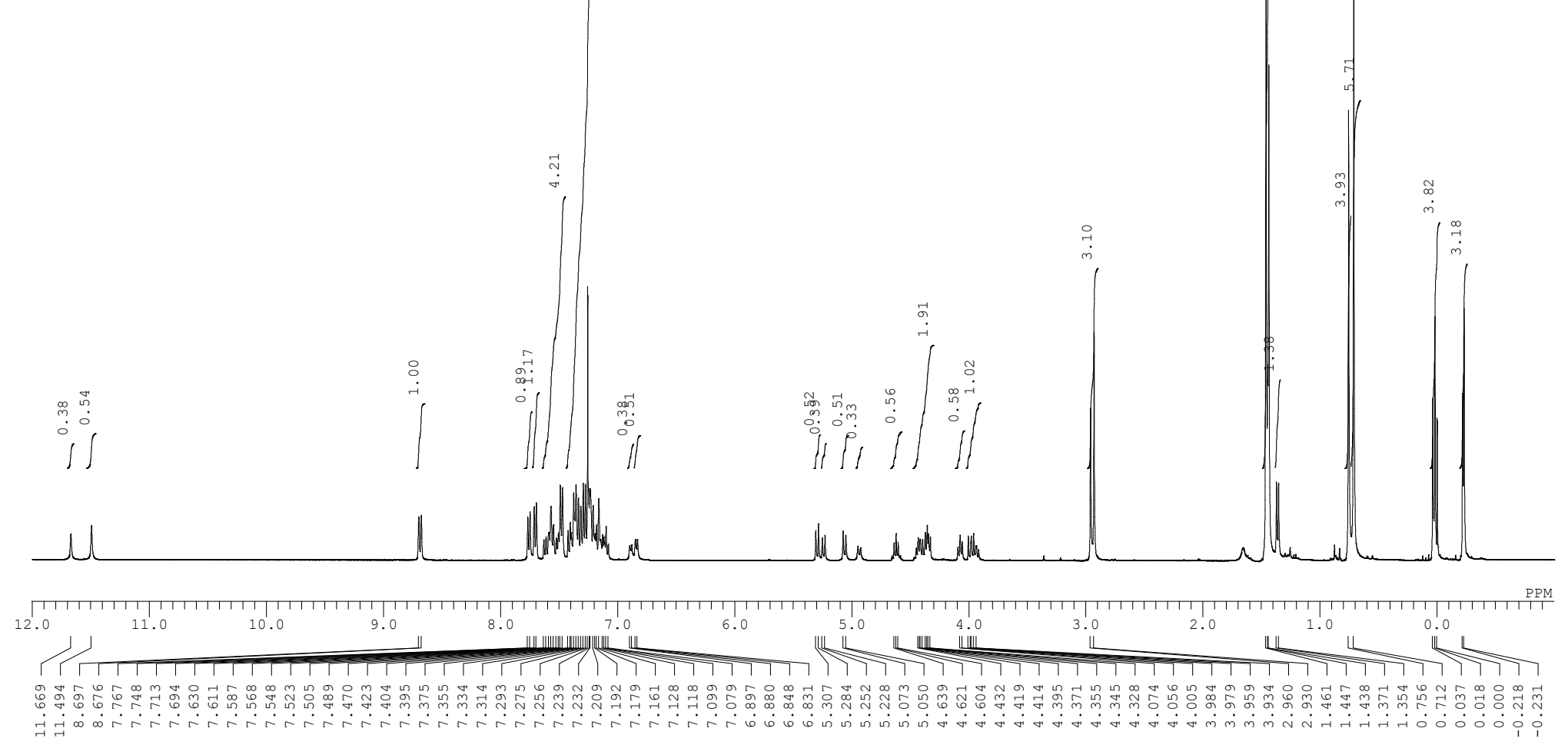




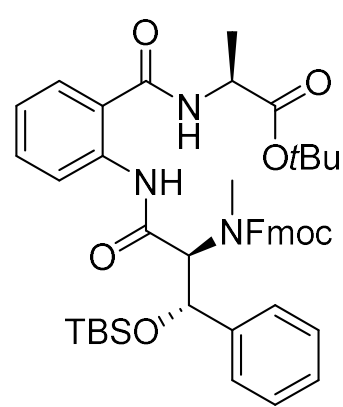

${ }^{13} \mathrm{C}$ NMR spectrum of $\mathbf{1 1 b}$

$11 \mathrm{~b}$

${ }^{13} \mathrm{C}$ NMR

$\left(100 \mathrm{MHz}\right.$ in $\left.\mathrm{CDCl}_{3}\right)$

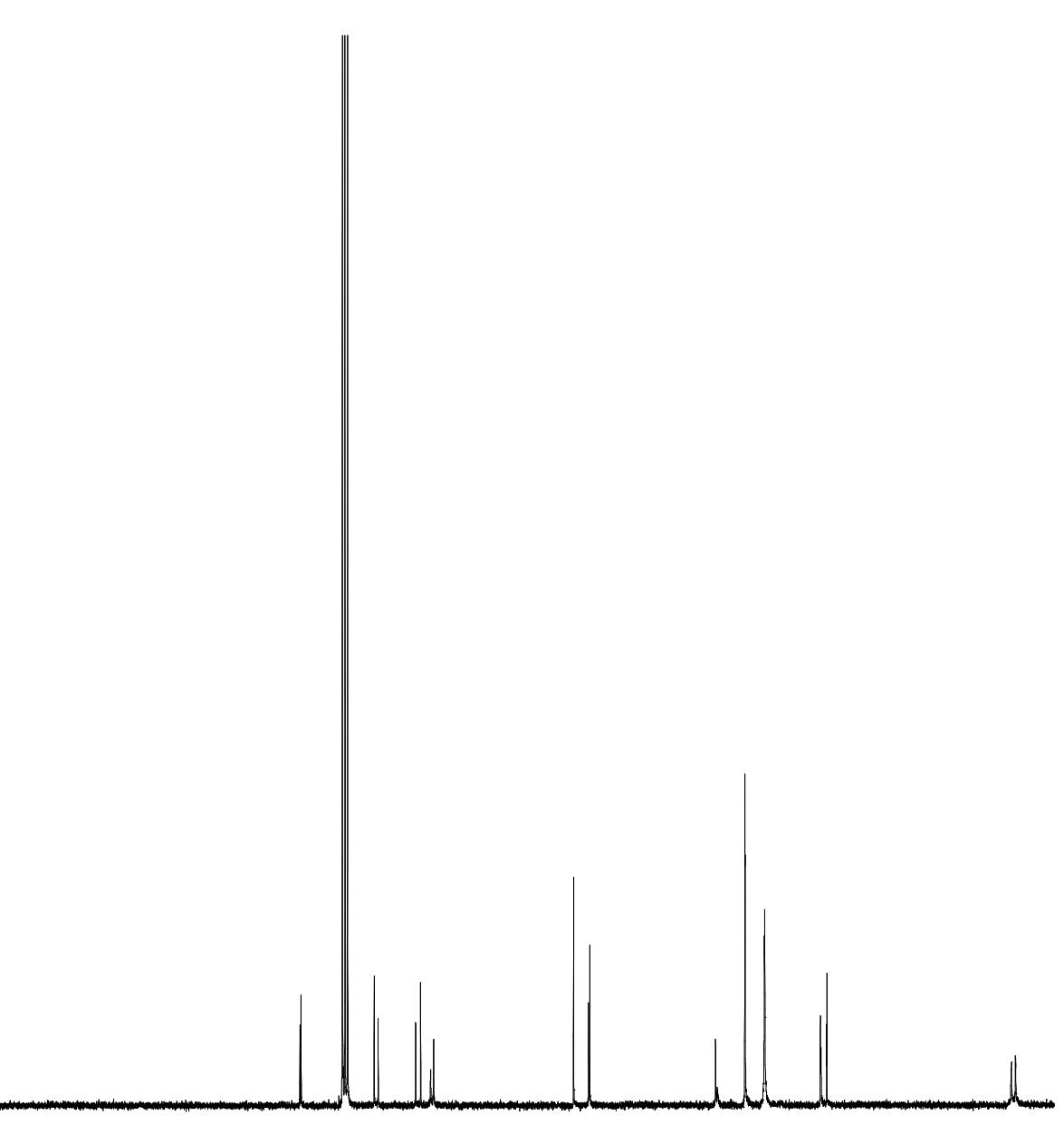

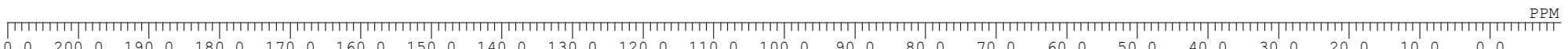

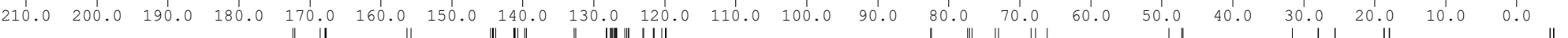

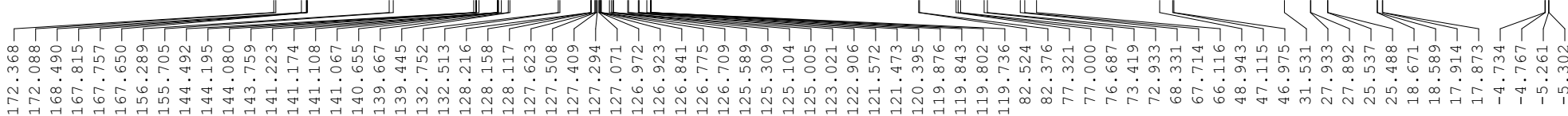




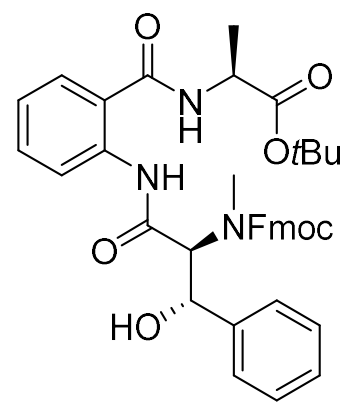

13

${ }^{1} \mathrm{H}$ NMR

(400 $\mathrm{MHz}$ in $\mathrm{CDCl}_{3}$ )

${ }^{1} \mathrm{H}$ NMR spectrum of $\mathbf{1 3}$
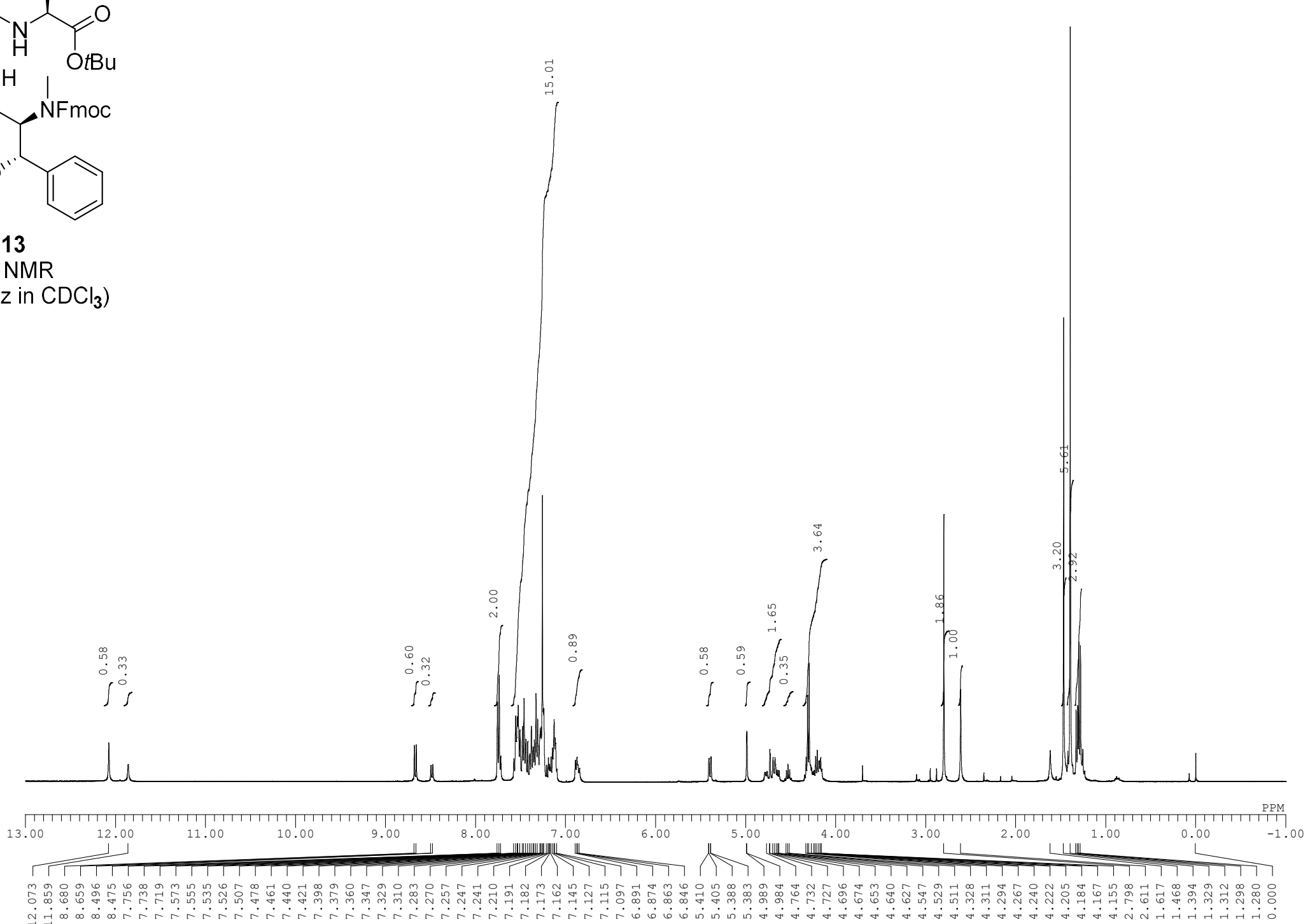


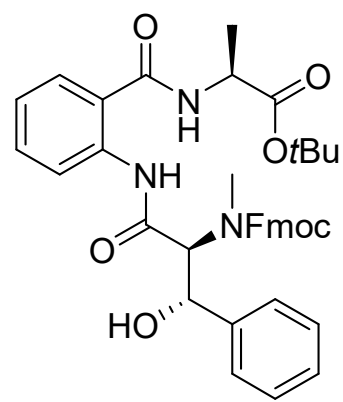

${ }^{13} \mathrm{C}$ NMR spectrum of $\mathbf{1 3}$

13

${ }^{13} \mathrm{C}$ NMR

$\left(100 \mathrm{MHz}\right.$ in $\mathrm{CDCl}_{3}$ )

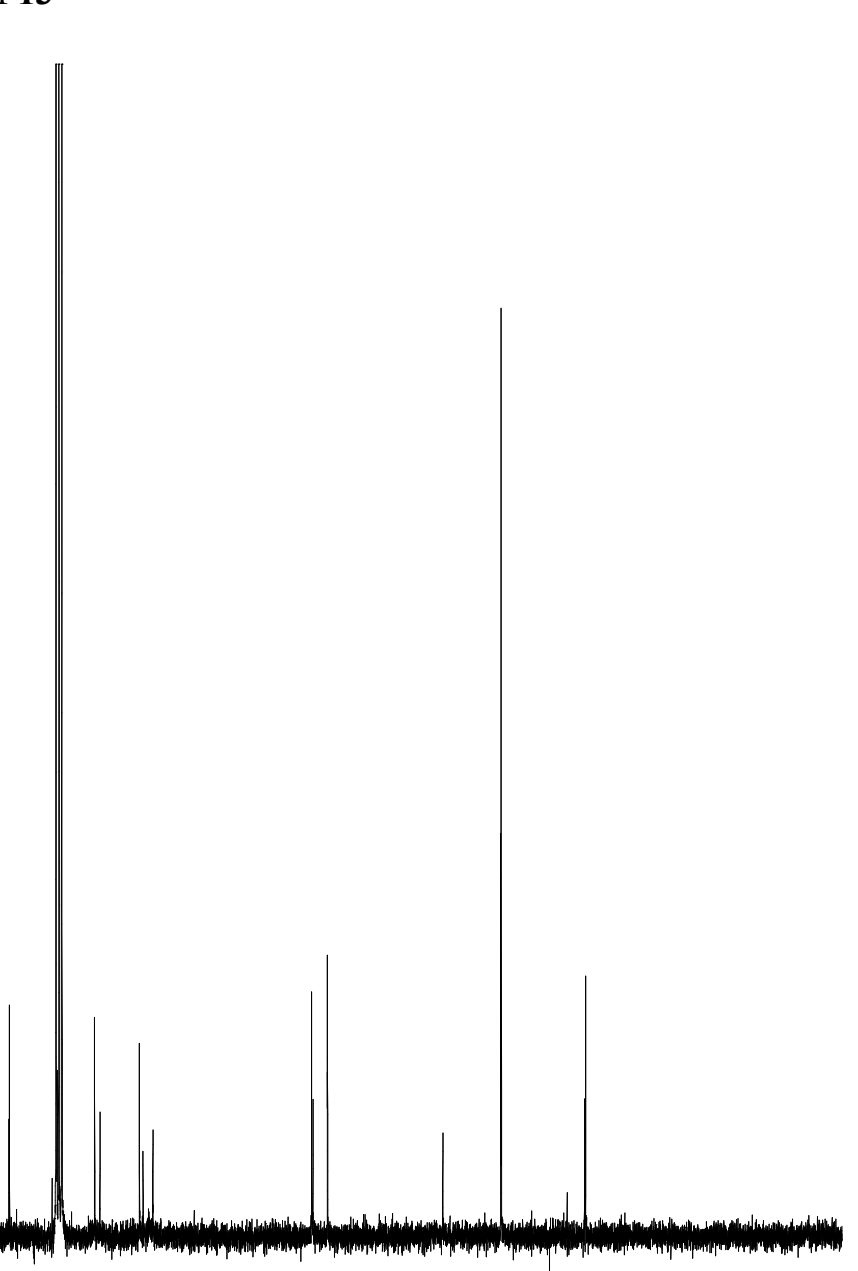

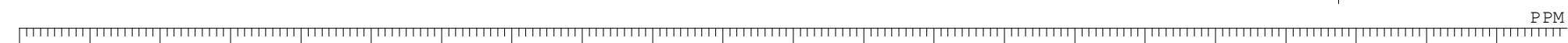

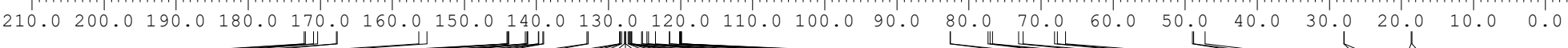

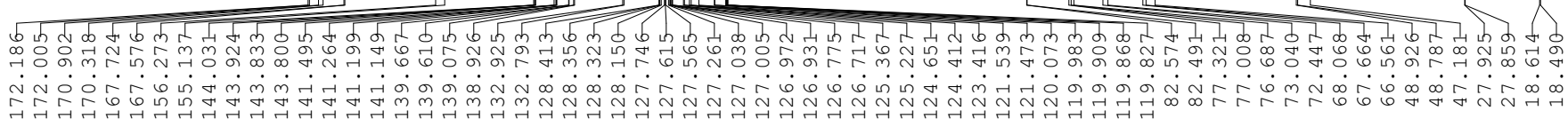




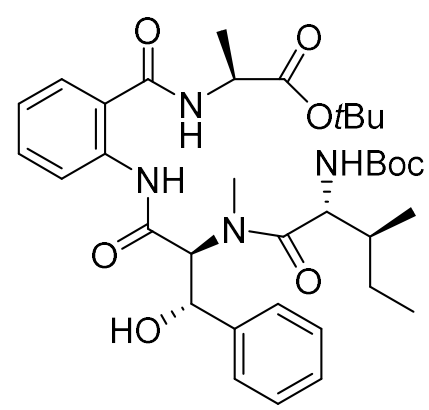

${ }^{1} \mathrm{H}$ NMR spectrum of $\mathbf{1 4}$

14

${ }^{1} \mathrm{H}$ NMR

(600 $\mathrm{MHz}$ in $\mathrm{CDCl}_{3}$ )

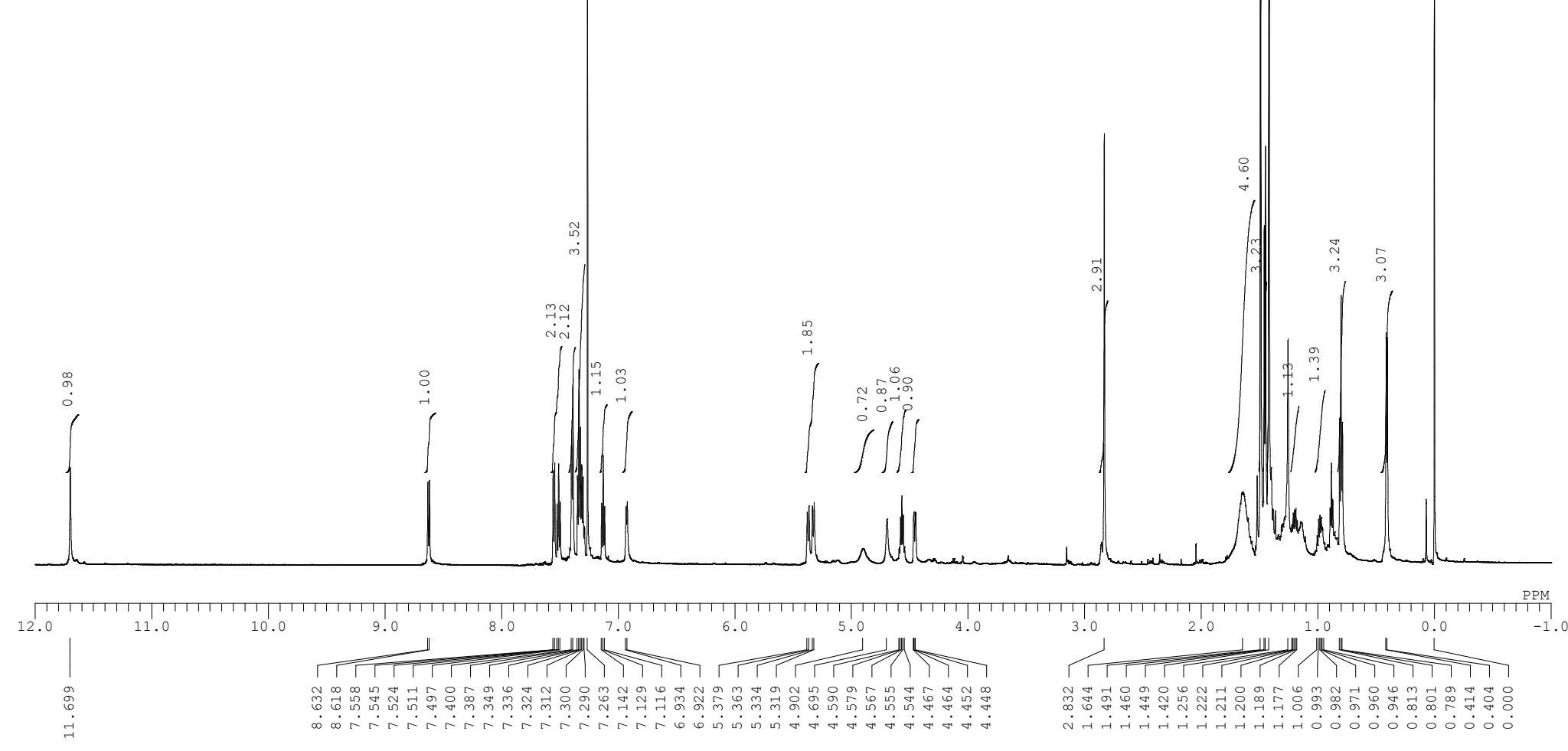




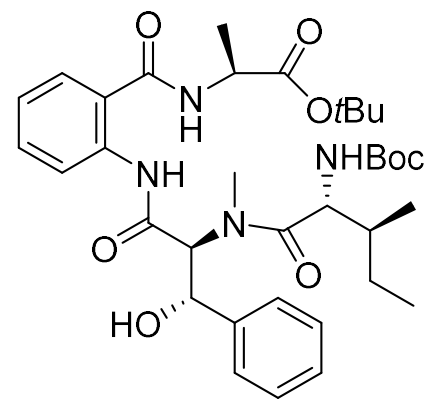

${ }^{13} \mathrm{C}$ NMR spectrum of $\mathbf{1 4}$

14

${ }^{13} \mathrm{C}$ NMR

$\left(150 \mathrm{MHz}\right.$ in $\left.\mathrm{CDCl}_{3}\right)$

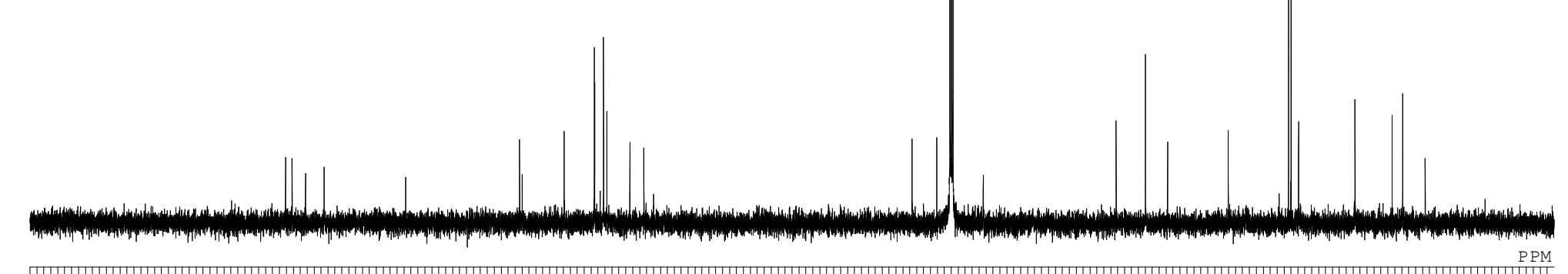

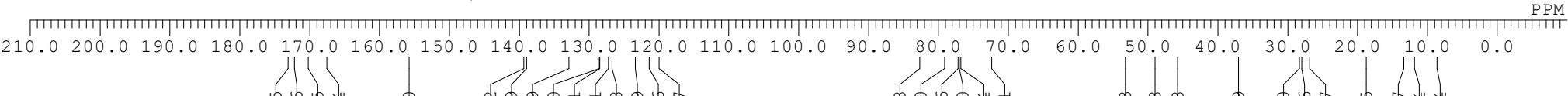

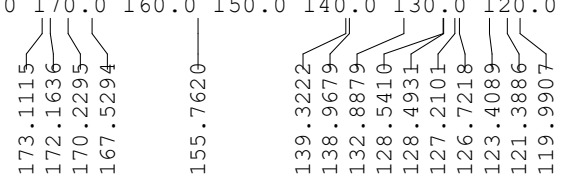




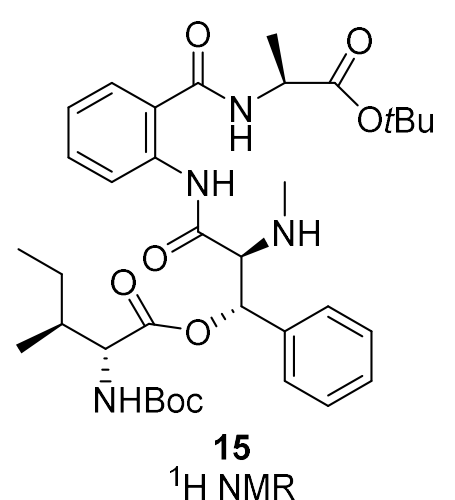

${ }^{1} \mathrm{H}$ NMR spectrum of $\mathbf{1 5}$

$\left(600 \mathrm{MHz}\right.$ in $\left.\mathrm{CDCl}_{3}\right)$

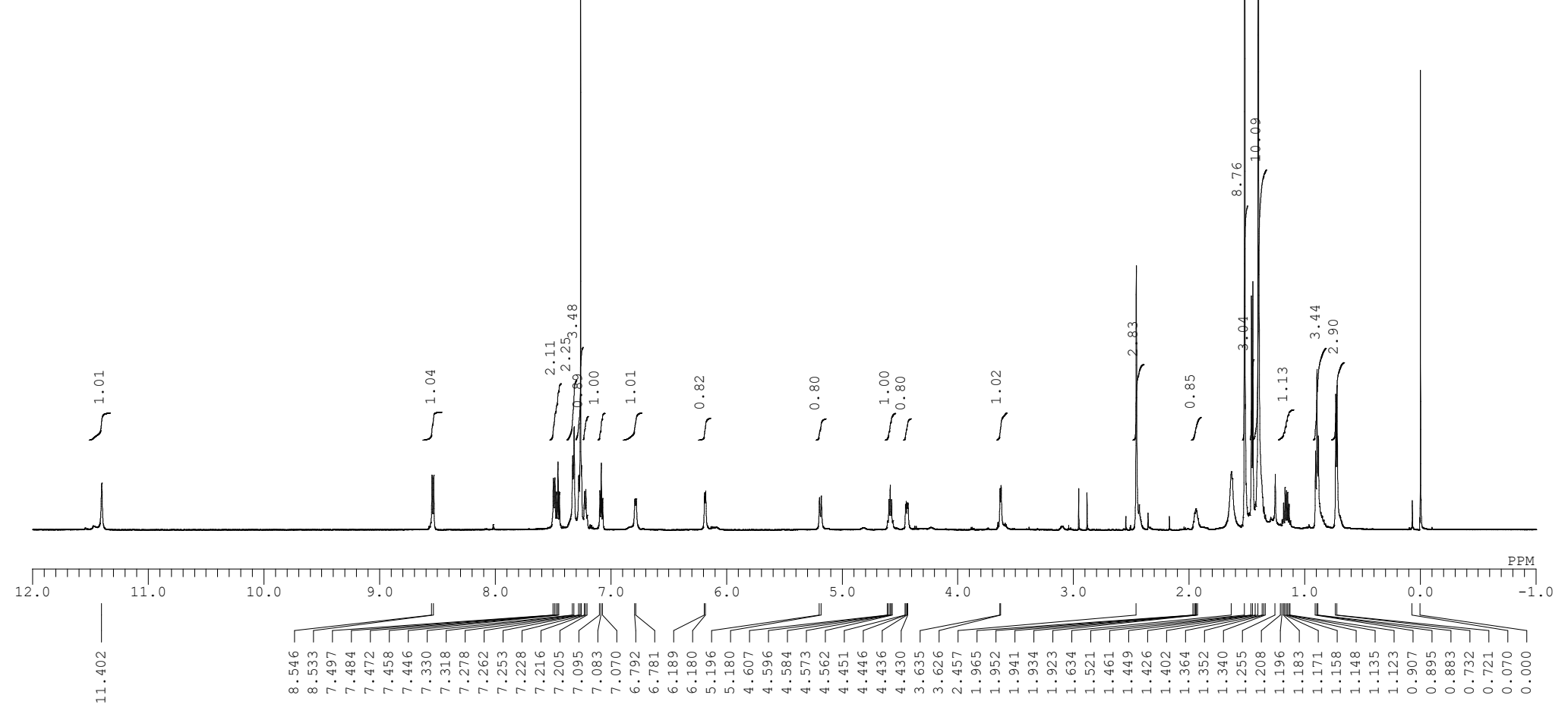




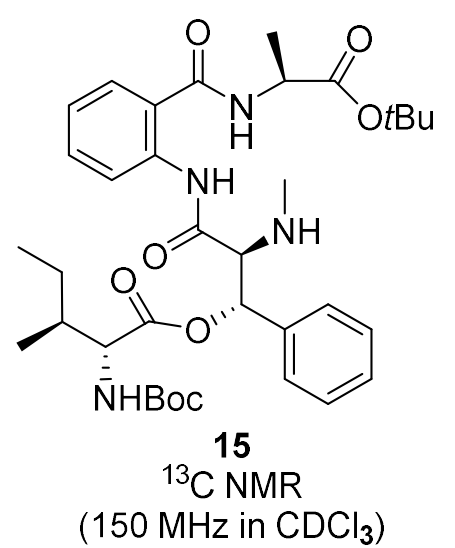

${ }^{13} \mathrm{C}$ NMR spectrum of $\mathbf{1 5}$

(150 MHz in $\mathrm{CDCl}_{3}$ )

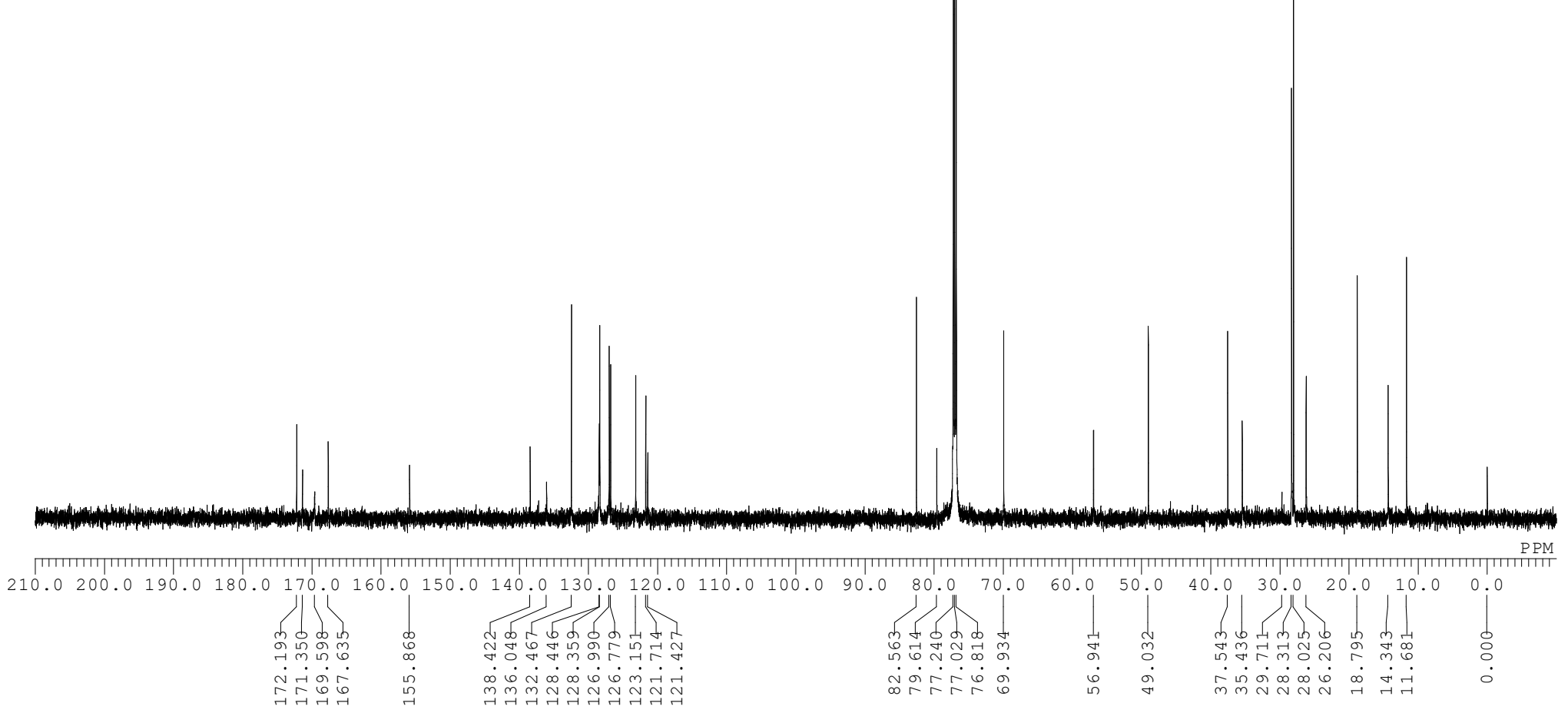




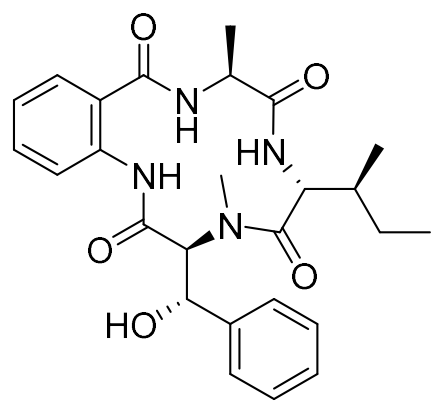

asperterrestide $A$

(1b, revised)

${ }^{1} \mathrm{H}$ NMR

$\left(600 \mathrm{MHz}\right.$ in $\left.\mathrm{CDCl}_{3}\right)$
${ }^{1} \mathrm{H}$ NMR spectrum of $\mathbf{1 b}$
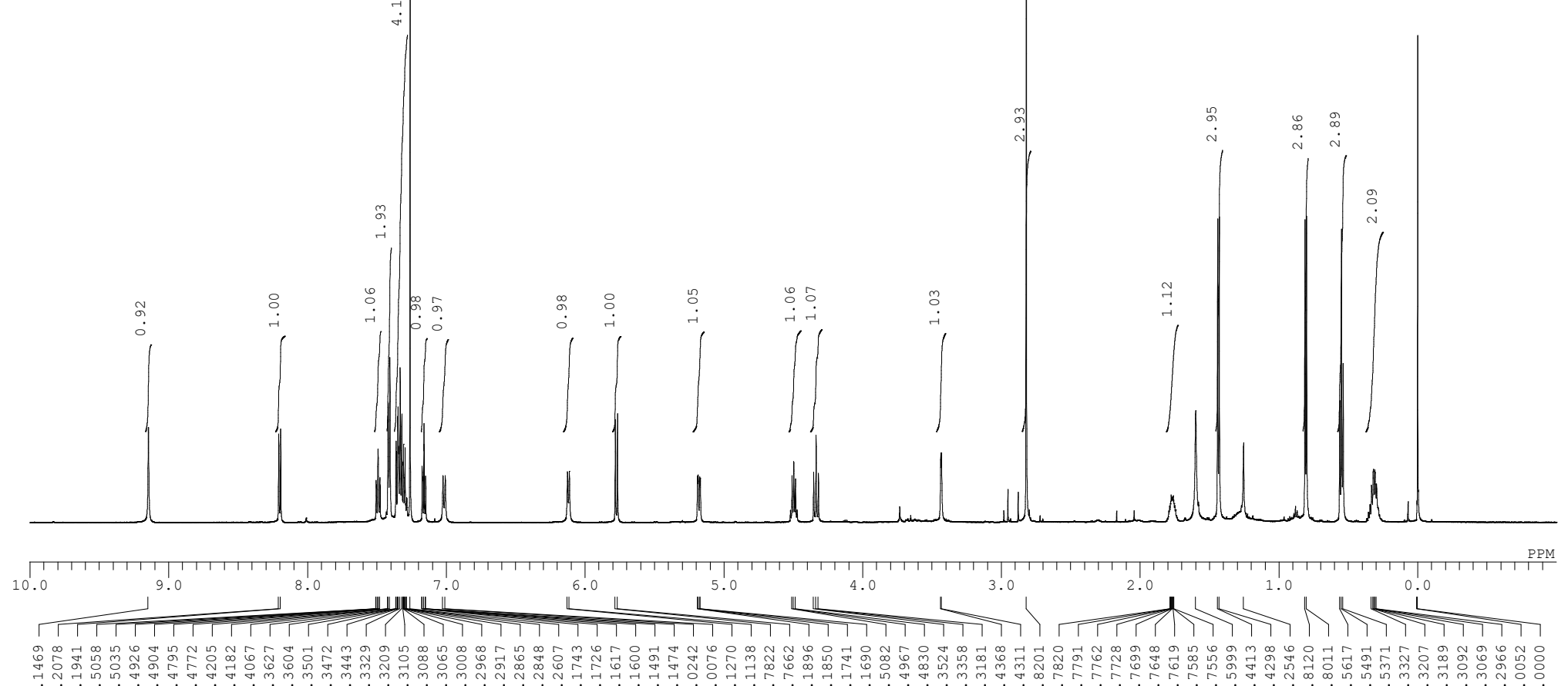

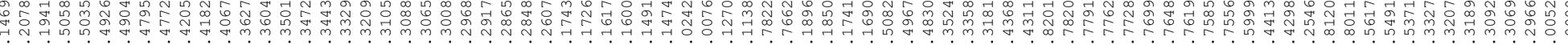




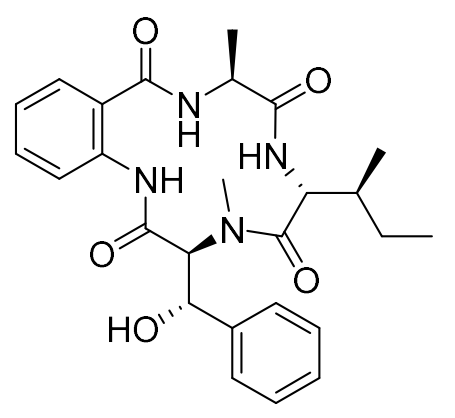

${ }^{13} \mathrm{C}$ NMR spectrum of $\mathbf{1 b}$

asperterrestide $A$

(1b, revised)

${ }^{13} \mathrm{C}$ NMR

(150 $\mathrm{MHz}$ in $\mathrm{CDCl}_{3}$ )

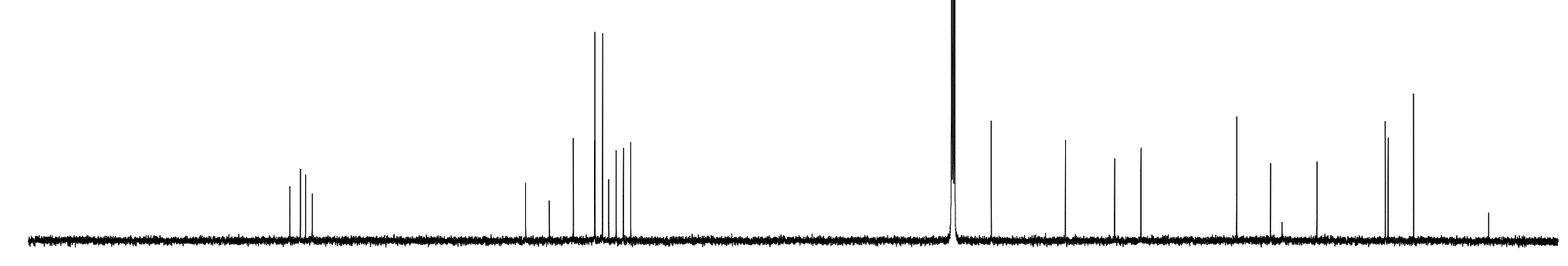

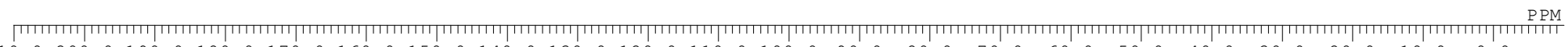
$210.0200 .0190 .0180 .0 \quad 170.0160 .0 \quad 150.0140 .0 \quad 130.0 \quad 120.0 \quad 110.0 \quad 100.0 \quad 90.0 \quad 80.0 \quad 70.0 \quad 60.0 \quad 50.0 \quad 40.0 \quad 30.0 \quad 20.0 \quad 10.0 \quad 0.0$
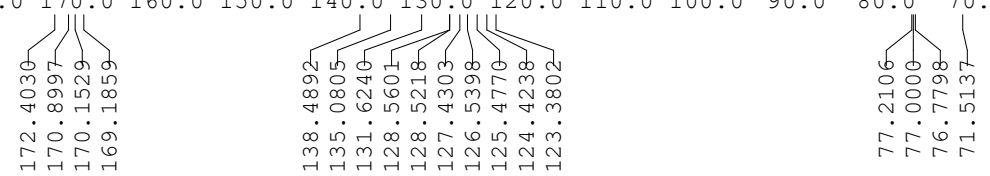
NOESY specutrum of the proposed structure of asperterrestide $\mathrm{A}(\mathbf{1 a})$ in $\mathrm{CDCl}_{3}$.

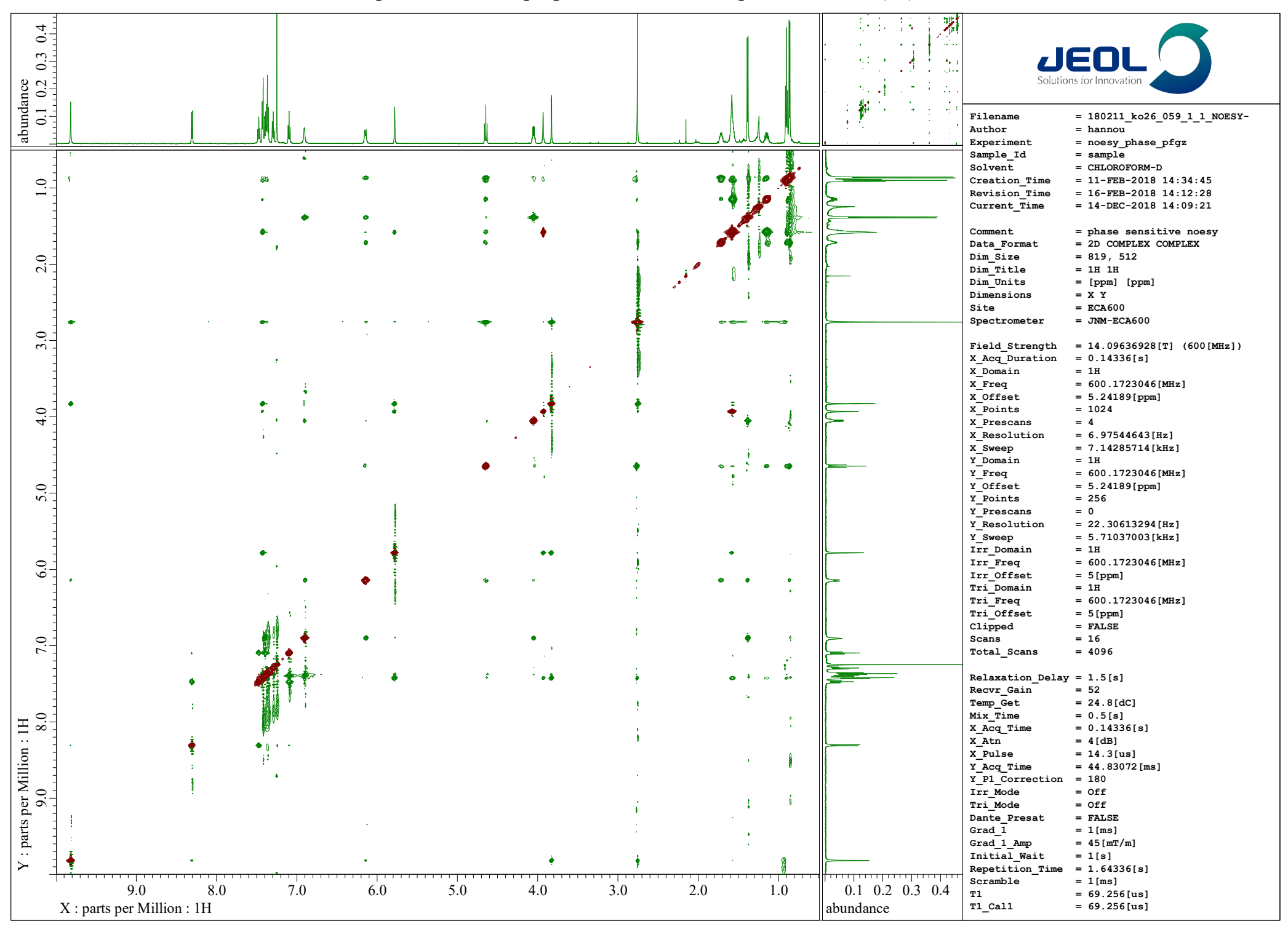


NOESY specutrum of the revised structure of asperterrestide $\mathrm{A}(\mathbf{1 b})$ in $\mathrm{CDCl}_{3}$.

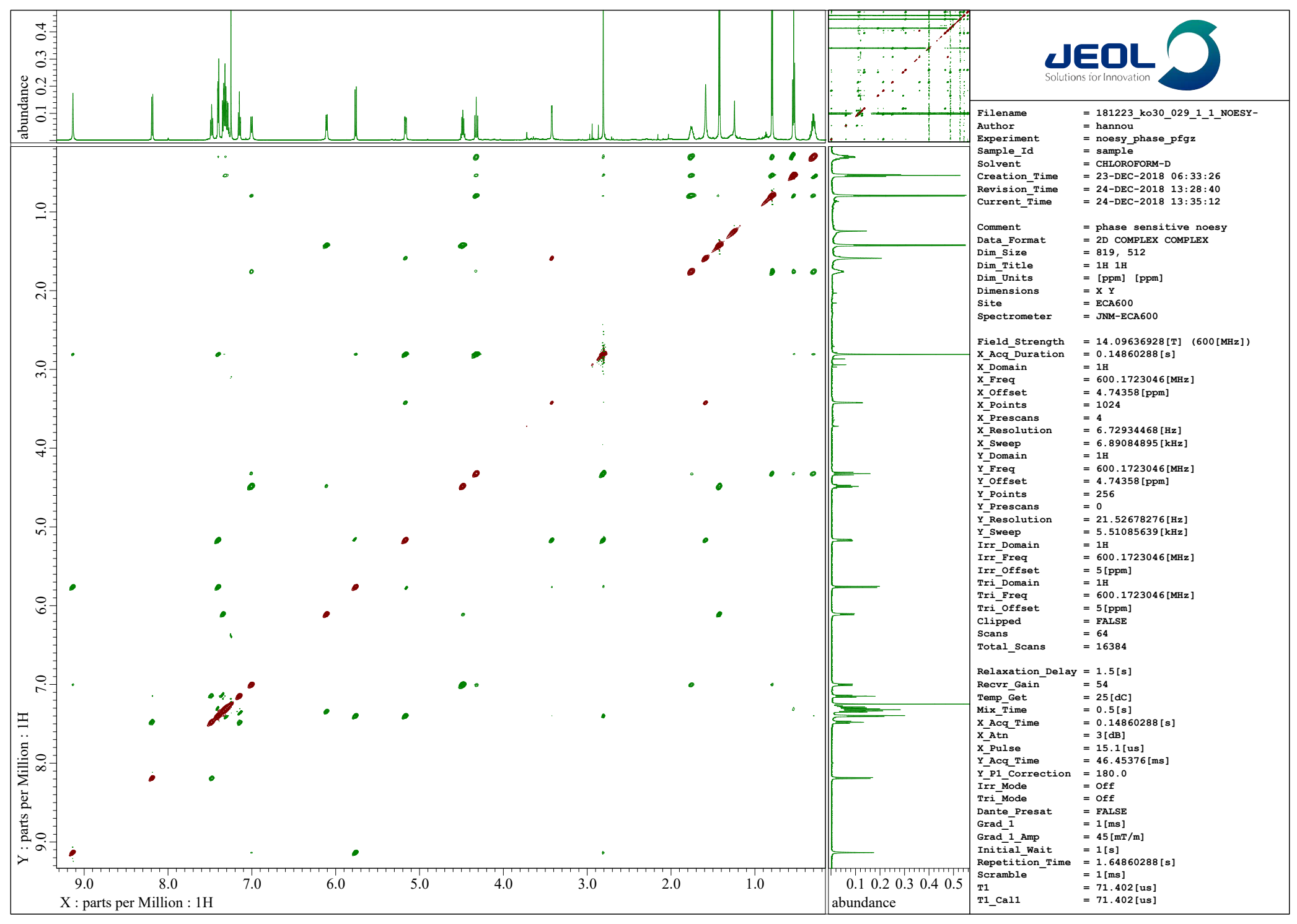


Table S1. Comparisons of NMR spectroscopic data observed in $\mathrm{CDCl}_{3}$ between natural asperterrestide A and synthetic 1a

\begin{tabular}{|c|c|c|c|c|c|c|c|}
\hline \multirow[b]{2}{*}{ Residue } & \multirow[b]{2}{*}{ Position } & \multicolumn{2}{|r|}{ Natural } & \multicolumn{2}{|c|}{ Synthetic 1a } & \multicolumn{2}{|c|}{$\begin{array}{l}\text { Deviations of } \\
\text { chemical shifts }\end{array}$} \\
\hline & & $\delta_{\mathrm{C}}$ & $\delta_{\mathrm{H}}(J$ in $\mathrm{Hz})$ & $\delta_{\mathrm{C}}$ & $\delta_{\mathrm{H}}(J$ in $\mathrm{Hz})$ & $\Delta \delta_{\mathrm{C}}^{\mathrm{a}}$ & $\Delta \delta_{\mathrm{H}}^{\mathrm{a}}$ \\
\hline \multirow[t]{7}{*}{ D-allo-Ile } & $\alpha$ & 53.9 & $4.31, \mathrm{dd}(10.5,10.0)$ & 53.8 & $4.66, \mathrm{t}(9.5)$ & -0.1 & 0.35 \\
\hline & $\beta$ & 36.1 & $1.75, \mathrm{~m}$ & 37.3 & $1.74, \mathrm{~m}$ & 1.2 & -0.01 \\
\hline & $\gamma$ & 24.7 & $0.30, \mathrm{~m}$ & 25.5 & $\begin{array}{l}1.59, \mathrm{~m} \\
1.16, \mathrm{~m}\end{array}$ & 0.8 & $\begin{array}{l}1.29 \\
0.86\end{array}$ \\
\hline & $\gamma^{\prime}$ & 14.5 & $0.79, \mathrm{~d}(6.5)$ & 14.3 & $0.87, \mathrm{~d}(6.8)$ & -0.2 & 0.08 \\
\hline & $\delta$ & 10.8 & $0.54, \mathrm{dd}(7.5,7.0)$ & 11.1 & $0.91, \mathrm{t}(7.4)$ & 0.3 & 0.37 \\
\hline & $\mathrm{C}=\mathrm{O}$ & 172.5 & & 174.9 & & 2.4 & \\
\hline & $\mathrm{NH}$ & & $7.18, \mathrm{~d}(9.5)$ & & $6.16, \mathrm{~d}(9.5)$ & & -1.02 \\
\hline \multirow{9}{*}{$\begin{array}{l}(2 R, 3 S)- \\
\text { MePhe(3-OH) }\end{array}$} & $\alpha$ & 60.9 & $5.76, \mathrm{~d}(10.0)$ & 72.9 & $3.84, \mathrm{~d}(2.6)$ & 12.0 & -1.92 \\
\hline & $\beta$ & 71.4 & $5.15, \mathrm{~d}(10.0)$ & 72.5 & 5.79, brs & 1.1 & 0.64 \\
\hline & $\mathrm{C} 1$ & 138.7 & & 141.2 & & 2.5 & \\
\hline & $\mathrm{C} 2 / \mathrm{C} 6$ & 127.5 & $7.39^{\mathrm{b}}$ & 125.88 & $7.44, \mathrm{~d}(7.3)$ & -1.6 & 0.05 \\
\hline & $\mathrm{C} 3 / \mathrm{C} 5$ & 128.5 & $7.33^{\mathrm{b}}$ & 128.6 & $7.38, \mathrm{t}(7.3)$ & 0.1 & 0.05 \\
\hline & $\mathrm{C} 4$ & 131.4 & $7.26^{\mathrm{b}}$ & 127.8 & $7.31, \mathrm{t}(7.3)$ & -3.6 & 0.05 \\
\hline & $\mathrm{C}=\mathrm{O}$ & 169.1 & & 166.5 & & -3.6 & \\
\hline & $\mathrm{N}-\mathrm{CH}_{3}$ & 31.4 & $2.75, \mathrm{~s}$ & 40.5 & $2.77, \mathrm{~s}$ & 9.1 & 0.02 \\
\hline & $\mathrm{OH}$ & & & & 3.94 , brs & & \\
\hline \multirow[t]{2}{*}{ Ant } & $\mathrm{C} 1$ & 135.1 & & 136.7 & & 1.6 & \\
\hline & $\mathrm{C} 2$ & 126.7 & & 123.7 & & -3.0 & \\
\hline
\end{tabular}




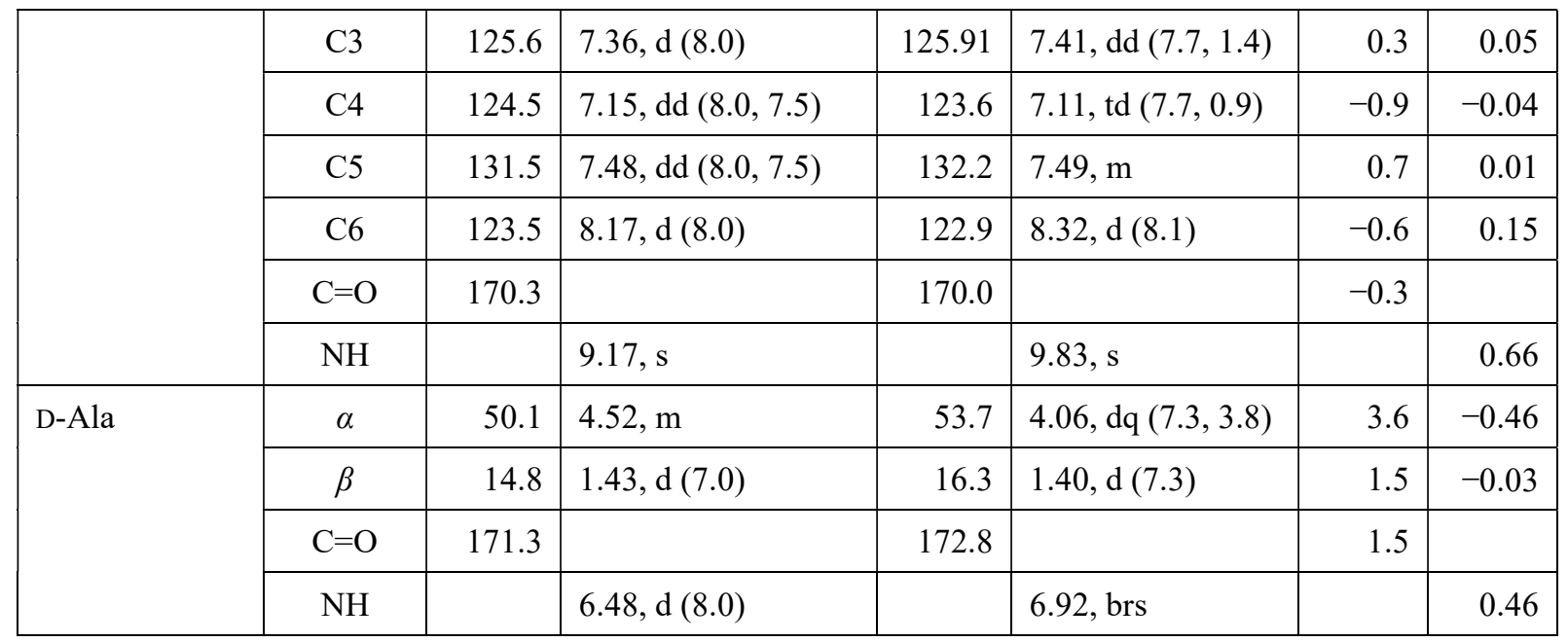

${ }^{\mathrm{a}} \Delta \delta(\mathrm{ppm})=\delta_{\text {synthetic }}(\mathrm{ppm})-\delta_{\text {natural }}(\mathrm{ppm}) .{ }^{\mathrm{b}}$ Overlapped signals. 
Table S2. Comparisons of NMR spectroscopic data observed in $\mathrm{CDCl}_{3}$ between natural asperterrestide $\mathrm{A}$ and synthetic $1 \mathrm{~b}$

\begin{tabular}{|c|c|c|c|c|c|c|c|}
\hline \multirow[b]{2}{*}{ Residue } & \multirow[b]{2}{*}{ Position } & \multicolumn{2}{|r|}{ Natural } & \multicolumn{2}{|r|}{ Synthetic 1b } & \multicolumn{2}{|c|}{$\begin{array}{l}\text { Deviations of } \\
\text { chemical shifts }\end{array}$} \\
\hline & & $\delta_{\mathrm{C}}$ & $\delta_{\mathrm{H}}(J$ in $\mathrm{Hz})$ & $\delta_{\mathrm{C}}$ & $\delta_{\mathrm{H}}(J$ in $\mathrm{Hz})$ & $\Delta \delta_{\mathrm{C}}^{\mathrm{a}}$ & $\Delta \delta_{\mathrm{H}}^{\mathrm{a}}$ \\
\hline \multirow[t]{7}{*}{ D-allo-Ile } & $\alpha$ & 53.9 & $4.31, \mathrm{dd}(10.5,10.0)$ & 53.8 & $4.34, \mathrm{t},(10.0)$ & -0.1 & 0.03 \\
\hline & $\beta$ & 36.1 & $1.75, \mathrm{~m}$ & 36.2 & $1.77, \mathrm{~m}$ & 0.1 & 0.02 \\
\hline & $\gamma$ & 24.7 & $0.30, \mathrm{~m}$ & 24.7 & $0.32, \mathrm{~m}$ & 0 & 0.02 \\
\hline & $\gamma^{\prime}$ & 14.5 & $0.79, \mathrm{~d}(6.5)$ & 14.4 & $0.81 \mathrm{~d}(6.5)$ & -0.1 & 0.02 \\
\hline & $\delta$ & 10.8 & $0.54, \mathrm{dd}(7.5,7.0)$ & 10.8 & $0.55, \mathrm{t}(7.4)$ & 0 & 0.01 \\
\hline & $\mathrm{C}=\mathrm{O}$ & 172.5 & & 172.4 & & -0.1 & \\
\hline & $\mathrm{NH}$ & & $7.18, \mathrm{~d}(9.5)$ & & $7.02, \mathrm{~d}(10.0)$ & & -0.16 \\
\hline \multirow{9}{*}{$\begin{array}{l}(2 S, 3 S)- \\
\text { MePhe(3-OH) }\end{array}$} & $\alpha$ & 60.9 & $5.76, \mathrm{~d}(10.0)$ & 60.8 & $5.77, \mathrm{~d}(9.5)$ & -0.1 & 0.01 \\
\hline & $\beta$ & 71.4 & $5.15, \mathrm{~d}(10.0)$ & 71.5 & 5.18, dd $(9.5,3.2)$ & 0.1 & 0.03 \\
\hline & $\mathrm{C} 1$ & 138.7 & & 138.5 & & -0.2 & \\
\hline & $\mathrm{C} 2 / \mathrm{C} 6$ & 127.5 & $7.39^{b}$ & 128.5 & $7.41, \mathrm{~m}$ & 1.0 & 0.02 \\
\hline & $\mathrm{C} 3 / \mathrm{C} 5$ & 128.5 & $7.33^{b}$ & 128.6 & $7.33^{\mathrm{b}}, \mathrm{m}$ & 0.1 & 0.00 \\
\hline & $\mathrm{C} 4$ & 127.6 & $7.26^{\mathrm{b}}$ & 127.4 & $7.30^{\mathrm{b}}, \mathrm{m}$ & -0.2 & 0.04 \\
\hline & $\mathrm{C}=\mathrm{O}$ & 169.1 & & 169.2 & & 0.1 & \\
\hline & $\mathrm{N}-\mathrm{CH}_{3}$ & 31.4 & $2.75, \mathrm{~s}$ & 31.3 & $2.82, \mathrm{~s}$ & -0.1 & 0.07 \\
\hline & $\mathrm{OH}$ & & & & $3.43, \mathrm{~d}(3.2)$ & & \\
\hline \multirow[t]{3}{*}{ Ant } & $\mathrm{C} 1$ & 135.1 & & 135.1 & & 0 & \\
\hline & $\mathrm{C} 2$ & 126.7 & & 126.5 & & -0.2 & \\
\hline & $\mathrm{C} 3$ & 125.6 & $7.36, \mathrm{~d}(8.0)$ & 125.5 & $7.35^{\mathrm{b}}, \mathrm{m}$ & -0.1 & -0.01 \\
\hline
\end{tabular}




\begin{tabular}{|c|c|c|c|c|c|c|c|}
\hline & $\mathrm{C} 4$ & 124.5 & $7.15, \mathrm{dd}(8.0,7.5)$ & 124.4 & $7.16, \mathrm{td},(7.9,1.0)$ & -0.1 & 0.01 \\
\hline & $\mathrm{C} 5$ & 131.5 & $7.48, \mathrm{dd}(8.0,7.5)$ & 131.6 & $7.49, \operatorname{td}(7.9,1.4)$ & 0.1 & 0.01 \\
\hline & C6 & 123.5 & $8.17, \mathrm{~d}(8.0)$ & 123.4 & 8.20, brd (7.9) & -0.1 & 0.03 \\
\hline & $\mathrm{C}=\mathrm{O}$ & 170.3 & & 170.2 & & -0.1 & \\
\hline & NH & & $9.17, \mathrm{~s}$ & & $9.15, \mathrm{~s}$ & & -0.02 \\
\hline \multirow[t]{4}{*}{ Ala } & $\alpha$ & 50.1 & $4.52, \mathrm{~m}$ & 50.0 & $4.49, \mathrm{~m}$ & -0.1 & -0.03 \\
\hline & $\beta$ & 14.8 & $1.43, \mathrm{~d}(7.0)$ & 14.8 & $1.44, \mathrm{~d}(6.9)$ & 0 & 0.01 \\
\hline & $\mathrm{C}=\mathrm{O}$ & 171.3 & & 170.9 & & -0.4 & \\
\hline & NH & & $6.48, \mathrm{~d}(8.0)$ & & $6.12, \mathrm{~d}(7.9)$ & & -0.36 \\
\hline
\end{tabular}

${ }^{\mathrm{a}} \Delta \delta(\mathrm{ppm})=\delta_{\text {synthetic }}(\mathrm{ppm})-\delta_{\text {natural }}(\mathrm{ppm}) .{ }^{\mathrm{b}}$ Overlapped signals 
3D structures, calculated total energies, relative energies and the atomic coordinates of $\mathbf{1 d}$

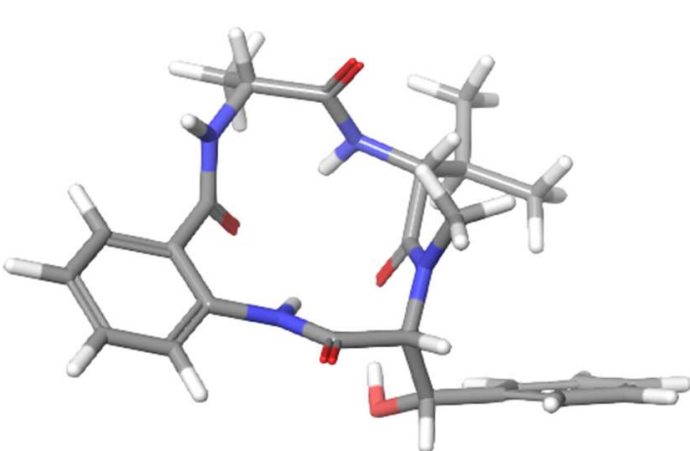

$\# 1$

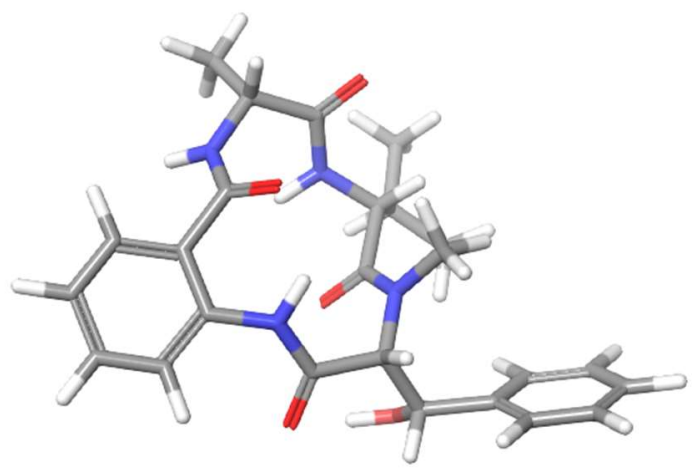

\#2

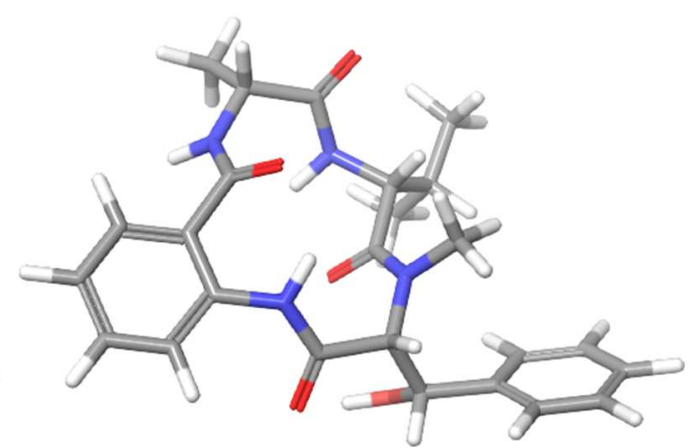

\#3

Figure S1. 3D structures of the conformers of $\mathbf{1 d}$

Table S3. Total and relative energies for conformers of $1 \mathrm{~d}$

\begin{tabular}{|c|c|c|c|}
\hline No. & $\begin{array}{c}\text { Relative Energy } \\
/ \mathrm{kJ} \bullet \mathrm{mol}^{-1}\end{array}$ & $\begin{array}{c}\text { Total Energy } \\
/ \mathrm{kJ}^{\prime} \mathrm{mol}^{-1}\end{array}$ & $\begin{array}{c}\text { Stretch (S), Bend (B), Torsional (T), Improper Torsional (IT), } \\
\text { Van der Waals (VDW), and Electrostatic (E) Energies / } \mathrm{kJ} \bullet \mathrm{mol}^{-1}\end{array}$ \\
\hline$\# 1$ & 0 & -24.1 & $\mathrm{~S}(15.47), \mathrm{B}(47.78), \mathrm{T}(94.90), \mathrm{IT}(3.81), \mathrm{VDW}(72.87), \mathrm{E}(-258.92)$ \\
\hline$\# 2$ & 2.8 & -21.3 & $\mathrm{~S}(16.19), \mathrm{B}(37.06), \mathrm{T}(85.74), \mathrm{IT}(3.34), \mathrm{VDW}(76.30), \mathrm{E}(-239.95)$ \\
\hline$\# 3$ & 4.7 & -19.4 & $\mathrm{~S}(16.86), \mathrm{B}(37.76), \mathrm{T}(90.12), \mathrm{IT}(4.13), \mathrm{VDW}(79.75), \mathrm{E}(-248.00)$ \\
\hline
\end{tabular}


Table S4. Cartesian coordinates of the conformers of $1 \mathrm{~d}$

$1 \mathrm{~d}$ \#1

\begin{tabular}{lrrr}
\hline atom & $\mathrm{x}$ & $\mathrm{y}$ & $\mathrm{z}$ \\
\hline $\mathrm{C}$ & 2.77160 & 5.78460 & -1.50820 \\
$\mathrm{C}$ & 3.70270 & 4.76730 & -1.19910 \\
$\mathrm{C}$ & 5.09170 & 4.99100 & -1.42620 \\
$\mathrm{C}$ & 5.51250 & 6.24610 & -1.92840 \\
$\mathrm{C}$ & 4.57530 & 7.25230 & -2.22650 \\
$\mathrm{C}$ & 3.20400 & 7.02150 & -2.02100 \\
$\mathrm{H}$ & 1.71700 & 5.62530 & -1.33450 \\
$\mathrm{H}$ & 6.56100 & 6.45150 & -2.09120 \\
$\mathrm{H}$ & 4.91370 & 8.20290 & -2.61260 \\
$\mathrm{H}$ & 2.48640 & 7.79740 & -2.24740 \\
$\mathrm{C}$ & 3.19710 & 3.46950 & -0.64060 \\
$\mathrm{O}$ & 3.65750 & 3.00200 & 0.39870 \\
$\mathrm{H}$ & 2.08020 & 3.27440 & -2.28460 \\
$\mathrm{~N}$ & 2.31160 & 2.82510 & -1.41220 \\
$\mathrm{C}$ & 1.83170 & 1.45030 & -1.24350 \\
$\mathrm{H}$ & 1.08420 & 1.29690 & -2.02330 \\
$\mathrm{O}$ & 3.09950 & 0.00440 & -2.67680 \\
$\mathrm{C}$ & 5.12730 & -0.44320 & -0.71240 \\
$\mathrm{C}$ & 6.14370 & 0.71430 & -0.53100 \\
$\mathrm{O}$ & 6.15940 & 1.27430 & 0.56610 \\
$\mathrm{~N}$ & 6.91980 & 1.14240 & -1.55990 \\
\hline & & &
\end{tabular}

\begin{tabular}{lrrr}
\hline $\mathrm{C}$ & 6.79200 & 0.54970 & -2.90080 \\
$\mathrm{H}$ & 7.11810 & -0.49140 & -2.89460 \\
$\mathrm{H}$ & 5.75930 & 0.59210 & -3.25170 \\
$\mathrm{C}$ & 7.14460 & 3.63100 & -1.83050 \\
$\mathrm{O}$ & 7.58190 & 4.28860 & -2.77450 \\
$\mathrm{~N}$ & 6.03550 & 3.96000 & -1.13990 \\
$\mathrm{H}$ & 5.77950 & 3.32920 & -0.38790 \\
$\mathrm{C}$ & 1.12520 & 1.21010 & 0.10850 \\
$\mathrm{H}$ & 0.73190 & 0.19410 & 0.16110 \\
$\mathrm{H}$ & 1.78700 & 1.34340 & 0.96420 \\
$\mathrm{H}$ & 0.28370 & 1.89120 & 0.23500 \\
$\mathrm{C}$ & 5.30360 & -1.52360 & 0.39480 \\
$\mathrm{H}$ & 5.32760 & -1.03400 & 1.37060 \\
$\mathrm{C}$ & 4.13110 & -2.52520 & 0.42550 \\
$\mathrm{H}$ & 4.00660 & -3.01870 & -0.53910 \\
$\mathrm{H}$ & 4.29310 & -3.29950 & 1.17590 \\
$\mathrm{H}$ & 3.18680 & -2.03940 & 0.67220 \\
$\mathrm{C}$ & 6.62670 & -2.29730 & 0.23620 \\
$\mathrm{H}$ & 6.75420 & -3.02750 & 1.03590 \\
$\mathrm{H}$ & 7.49210 & -1.63650 & 0.26980 \\
$\mathrm{H}$ & 6.66180 & -2.83590 & -0.71140 \\
$\mathrm{C}$ & 2.96890 & 0.46200 & -1.54540 \\
\hline & & &
\end{tabular}

\begin{tabular}{lrrr}
\hline $\mathrm{N}$ & 3.82830 & 0.20350 & -0.55470 \\
$\mathrm{H}$ & 3.71630 & 0.75410 & 0.28930 \\
$\mathrm{H}$ & 5.19840 & -0.94070 & -1.67830 \\
$\mathrm{C}$ & 7.84400 & 2.30750 & -1.46040 \\
$\mathrm{H}$ & 8.55410 & 2.17230 & -2.27620 \\
$\mathrm{C}$ & 8.79740 & 2.34630 & -0.22040 \\
$\mathrm{H}$ & 9.58400 & 3.06270 & -0.46550 \\
$\mathrm{O}$ & 8.20380 & 2.90330 & 0.93810 \\
$\mathrm{H}$ & 7.45620 & 2.34870 & 1.15620 \\
$\mathrm{C}$ & 10.90200 & -1.40380 & 0.49580 \\
$\mathrm{C}$ & 11.10290 & -0.69170 & -0.70140 \\
$\mathrm{C}$ & 10.42460 & 0.52280 & -0.92160 \\
$\mathrm{C}$ & 9.53430 & 1.03380 & 0.04860 \\
$\mathrm{C}$ & 9.34360 & 0.31420 & 1.25140 \\
$\mathrm{C}$ & 10.02500 & -0.89830 & 1.47350 \\
$\mathrm{H}$ & 11.42580 & -2.33310 & 0.66770 \\
$\mathrm{H}$ & 11.78280 & -1.07450 & -1.44900 \\
$\mathrm{H}$ & 10.59530 & 1.06300 & -1.84160 \\
$\mathrm{H}$ & 8.67490 & 0.68470 & 2.01490 \\
$\mathrm{H}$ & 9.87410 & -1.43940 & 2.39650 \\
$\mathrm{H}$ & 7.38340 & 1.06410 & -3.65870 \\
\hline & & &
\end{tabular}




\section{$1 d \# 2$}

\begin{tabular}{lrrr}
\hline atom & $\mathrm{x}$ & $\mathrm{y}$ & $\mathrm{z}$ \\
\hline $\mathrm{C}$ & 2.74340 & 5.55660 & -1.82790 \\
$\mathrm{C}$ & 3.74430 & 4.56050 & -1.90760 \\
$\mathrm{C}$ & 5.10010 & 4.90930 & -1.63320 \\
$\mathrm{C}$ & 5.41810 & 6.24610 & -1.29430 \\
$\mathrm{C}$ & 4.40900 & 7.22280 & -1.21260 \\
$\mathrm{C}$ & 3.07200 & 6.87950 & -1.47840 \\
$\mathrm{H}$ & 1.71410 & 5.31610 & -2.05220 \\
$\mathrm{H}$ & 6.43820 & 6.54160 & -1.09620 \\
$\mathrm{H}$ & 4.66610 & 8.23890 & -0.95010 \\
$\mathrm{H}$ & 2.30090 & 7.63490 & -1.42320 \\
$\mathrm{C}$ & 3.35690 & 3.15940 & -2.30200 \\
$\mathrm{O}$ & 4.06380 & 2.50030 & -3.06450 \\
$\mathrm{H}$ & 1.78720 & 3.25460 & -1.04430 \\
$\mathrm{~N}$ & 2.27500 & 2.65380 & -1.68880 \\
$\mathrm{C}$ & 1.79350 & 1.27410 & -1.84870 \\
$\mathrm{H}$ & 1.60030 & 1.10030 & -2.90940 \\
$\mathrm{O}$ & 2.90970 & -0.85380 & -1.96650 \\
$\mathrm{C}$ & 4.88000 & -0.08040 & -0.01670 \\
$\mathrm{C}$ & 5.89300 & 1.08790 & -0.01940 \\
$\mathrm{O}$ & 5.72340 & 1.95020 & 0.84500 \\
$\mathrm{~N}$ & 6.84120 & 1.20750 & -0.99270 \\
\hline & & &
\end{tabular}

\begin{tabular}{lrrr}
\hline $\mathrm{C}$ & 6.94870 & 0.22480 & -2.08090 \\
$\mathrm{H}$ & 7.72600 & 0.46330 & -2.80630 \\
$\mathrm{H}$ & 7.19710 & -0.76030 & -1.68240 \\
$\mathrm{C}$ & 7.20310 & 3.73020 & -0.96640 \\
$\mathrm{O}$ & 7.70590 & 4.61200 & -0.27110 \\
$\mathrm{~N}$ & 6.10390 & 3.90310 & -1.72220 \\
$\mathrm{H}$ & 5.82530 & 3.09510 & -2.26740 \\
$\mathrm{C}$ & 0.48180 & 1.10460 & -1.06660 \\
$\mathrm{H}$ & 0.09070 & 0.09250 & -1.18460 \\
$\mathrm{H}$ & 0.62040 & 1.27830 & 0.00130 \\
$\mathrm{H}$ & -0.28570 & 1.79050 & -1.42580 \\
$\mathrm{C}$ & 4.73540 & -0.78130 & 1.36510 \\
$\mathrm{H}$ & 4.45070 & -0.03020 & 2.10450 \\
$\mathrm{C}$ & 3.62890 & -1.85450 & 1.35970 \\
$\mathrm{H}$ & 3.82130 & -2.61720 & 0.60420 \\
$\mathrm{H}$ & 3.55610 & -2.35380 & 2.32620 \\
$\mathrm{H}$ & 2.64930 & -1.42510 & 1.14980 \\
$\mathrm{C}$ & 6.05800 & -1.40460 & 1.84890 \\
$\mathrm{H}$ & 5.92470 & -1.92770 & 2.79630 \\
$\mathrm{H}$ & 6.81880 & -0.64220 & 2.01740 \\
$\mathrm{H}$ & 6.45220 & -2.12040 & 1.12660 \\
$\mathrm{C}$ & 2.82060 & 0.22240 & -1.38460 \\
\hline
\end{tabular}

\begin{tabular}{lrrr}
\hline $\mathrm{N}$ & 3.62520 & 0.56790 & -0.37450 \\
$\mathrm{H}$ & 3.49450 & 1.47900 & 0.03910 \\
$\mathrm{H}$ & 5.13300 & -0.84730 & -0.74680 \\
$\mathrm{C}$ & 7.83850 & 2.31770 & -1.01690 \\
$\mathrm{H}$ & 8.32440 & 2.32730 & -1.99200 \\
$\mathrm{C}$ & 8.98320 & 2.08550 & 0.02170 \\
$\mathrm{H}$ & 9.67360 & 2.92760 & -0.05910 \\
$\mathrm{O}$ & 8.55420 & 2.10560 & 1.37210 \\
$\mathrm{H}$ & 8.04680 & 2.89790 & 1.50330 \\
$\mathrm{C}$ & 11.34570 & -1.43470 & -0.97110 \\
$\mathrm{C}$ & 11.50410 & -0.24310 & -1.70400 \\
$\mathrm{C}$ & 10.74500 & 0.89570 & -1.37060 \\
$\mathrm{C}$ & 9.82100 & 0.85180 & -0.30370 \\
$\mathrm{C}$ & 9.67260 & -0.34620 & 0.43020 \\
$\mathrm{C}$ & 10.43090 & -1.48540 & 0.09770 \\
$\mathrm{H}$ & 11.93000 & -2.30770 & -1.22410 \\
$\mathrm{H}$ & 12.21050 & -0.20220 & -2.52050 \\
$\mathrm{H}$ & 10.87510 & 1.80540 & -1.93860 \\
$\mathrm{H}$ & 8.97890 & -0.39060 & 1.25640 \\
$\mathrm{H}$ & 10.31310 & -2.39580 & 0.66720 \\
$\mathrm{H}$ & 6.01100 & 0.15300 & -2.63590 \\
\hline & & & \\
\hline
\end{tabular}


$1 d$ \#3

\begin{tabular}{lrrr}
\hline atom & $\mathrm{x}$ & $\mathrm{y}$ & $\mathrm{z}$ \\
\hline $\mathrm{C}$ & 2.75670 & 5.56450 & -1.68810 \\
$\mathrm{C}$ & 3.73660 & 4.55080 & -1.80680 \\
$\mathrm{C}$ & 5.11760 & 4.90260 & -1.72180 \\
$\mathrm{C}$ & 5.47590 & 6.25920 & -1.53300 \\
$\mathrm{C}$ & 4.48790 & 7.25250 & -1.40920 \\
$\mathrm{C}$ & 3.12800 & 6.90630 & -1.48490 \\
$\mathrm{H}$ & 1.70710 & 5.32220 & -1.77170 \\
$\mathrm{H}$ & 6.51330 & 6.55680 & -1.48290 \\
$\mathrm{H}$ & 4.77800 & 8.28260 & -1.26110 \\
$\mathrm{H}$ & 2.37110 & 7.67310 & -1.39870 \\
$\mathrm{C}$ & 3.29210 & 3.13270 & -2.05550 \\
$\mathrm{O}$ & 3.89940 & 2.41780 & -2.85300 \\
$\mathrm{H}$ & 1.88400 & 3.35490 & -0.64260 \\
$\mathrm{~N}$ & 2.26420 & 2.69840 & -1.30460 \\
$\mathrm{C}$ & 1.65700 & 1.35760 & -1.35700 \\
$\mathrm{H}$ & 1.21950 & 1.23820 & -2.35020 \\
$\mathrm{O}$ & 2.58080 & -0.80880 & -1.85110 \\
$\mathrm{C}$ & 5.00010 & -0.15650 & -0.40360 \\
$\mathrm{C}$ & 5.96040 & 1.05450 & -0.32870 \\
$\mathrm{O}$ & 5.69200 & 1.91340 & 0.51650 \\
$\mathrm{~N}$ & 6.98030 & 1.20950 & -1.21840 \\
\hline & & &
\end{tabular}

\begin{tabular}{lrrr}
\hline $\mathrm{C}$ & 7.24520 & 0.21180 & -2.26570 \\
$\mathrm{H}$ & 6.45070 & 0.21770 & -3.01400 \\
$\mathrm{H}$ & 8.18530 & 0.38100 & -2.79080 \\
$\mathrm{C}$ & 7.26300 & 3.75770 & -1.15970 \\
$\mathrm{O}$ & 7.80090 & 4.68460 & -0.55470 \\
$\mathrm{~N}$ & 6.11080 & 3.88740 & -1.84580 \\
$\mathrm{H}$ & 5.78480 & 3.04840 & -2.31040 \\
$\mathrm{C}$ & 0.53260 & 1.26510 & -0.31450 \\
$\mathrm{H}$ & 0.05180 & 0.28600 & -0.35160 \\
$\mathrm{H}$ & 0.90950 & 1.40530 & 0.69940 \\
$\mathrm{H}$ & -0.24060 & 2.01170 & -0.49630 \\
$\mathrm{C}$ & 5.22090 & -1.23470 & 0.69480 \\
$\mathrm{H}$ & 6.26660 & -1.54040 & 0.62830 \\
$\mathrm{C}$ & 4.99090 & -0.73850 & 2.13720 \\
$\mathrm{H}$ & 3.96750 & -0.39410 & 2.28660 \\
$\mathrm{H}$ & 5.17650 & -1.53490 & 2.85820 \\
$\mathrm{H}$ & 5.66030 & 0.08260 & 2.39580 \\
$\mathrm{C}$ & 4.37890 & -2.49520 & 0.42940 \\
$\mathrm{H}$ & 4.62170 & -3.29020 & 1.13470 \\
$\mathrm{H}$ & 4.55250 & -2.88360 & -0.57480 \\
$\mathrm{H}$ & 3.31190 & -2.28910 & 0.51890 \\
$\mathrm{C}$ & 2.66830 & 0.21050 & -1.17330 \\
\hline
\end{tabular}

\begin{tabular}{lrrr}
\hline $\mathrm{N}$ & 3.67090 & 0.43430 & -0.32020 \\
$\mathrm{H}$ & 3.66110 & 1.30470 & 0.19360 \\
$\mathrm{H}$ & 5.08510 & -0.64380 & -1.37360 \\
$\mathrm{C}$ & 7.93240 & 2.35650 & -1.16170 \\
$\mathrm{H}$ & 8.50020 & 2.37530 & -2.09170 \\
$\mathrm{C}$ & 8.98370 & 2.15770 & -0.01970 \\
$\mathrm{H}$ & 9.70780 & 2.97110 & -0.09970 \\
$\mathrm{O}$ & 8.45350 & 2.28550 & 1.28800 \\
$\mathrm{H}$ & 8.00030 & 3.11820 & 1.33730 \\
$\mathrm{C}$ & 11.26640 & -1.50870 & -0.55450 \\
$\mathrm{C}$ & 11.59150 & -0.35700 & -1.29660 \\
$\mathrm{C}$ & 10.85790 & 0.83130 & -1.11210 \\
$\mathrm{C}$ & 9.79440 & 0.87590 & -0.18480 \\
$\mathrm{C}$ & 9.47630 & -0.28270 & 0.55910 \\
$\mathrm{C}$ & 10.20920 & -1.47100 & 0.37460 \\
$\mathrm{H}$ & 11.83140 & -2.41920 & -0.69320 \\
$\mathrm{H}$ & 12.40640 & -0.38490 & -2.00560 \\
$\mathrm{H}$ & 11.11470 & 1.70980 & -1.68620 \\
$\mathrm{H}$ & 8.66740 & -0.25790 & 1.27590 \\
$\mathrm{H}$ & 9.96240 & -2.35120 & 0.95030 \\
$\mathrm{H}$ & 7.32020 & -0.79050 & -1.84030 \\
\hline & & & \\
\hline
\end{tabular}


3D structures, calculated total energies, relative energies and the atomic coordinates of $1 \mathrm{e}$.

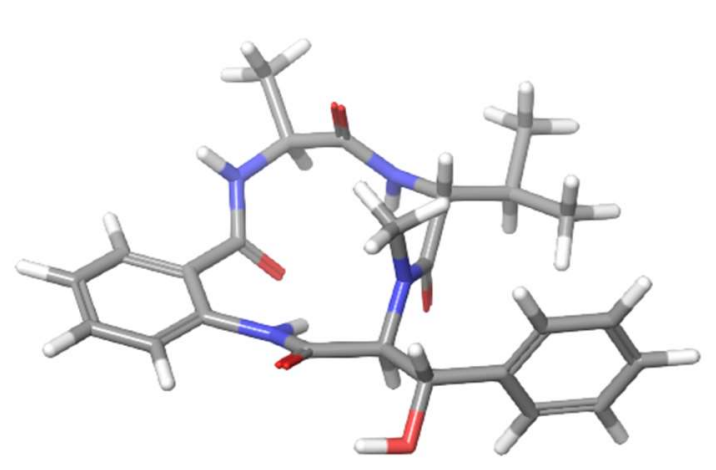

$\# 1$

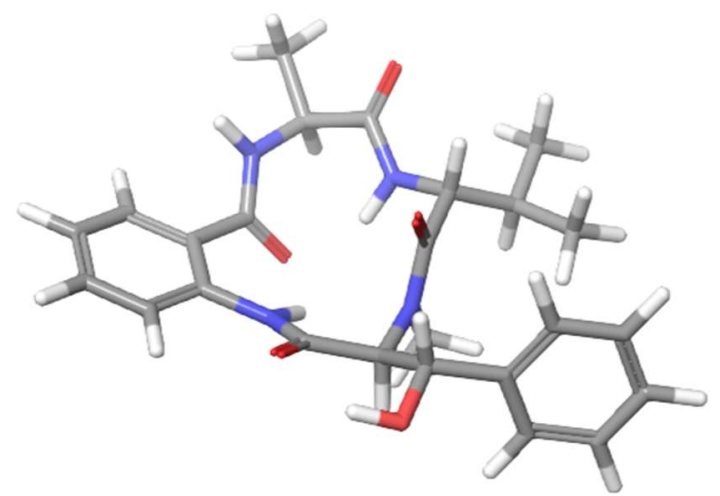

$\# 2$

Figure S2. 3D structures of the conformers of $\mathbf{1 e}$

\section{Table S5. Total and relative energies for conformers of $1 \mathrm{e}$}

\begin{tabular}{|c|c|c|c|}
\hline No. & $\begin{array}{l}\text { Relative Energy } \\
\qquad / \mathrm{kJ} \cdot \mathrm{mol}^{-1}\end{array}$ & $\begin{array}{l}\text { Total Energy } \\
\qquad / \mathrm{kJ} \bullet \mathrm{mol}^{-1}\end{array}$ & $\begin{array}{l}\text { Stretch (S), Bend (B), Torsional (T), Improper Torsional (IT), } \\
\text { Van der Waals (VDW), and Electrostatic (E) Energies } / \mathrm{kJ} \bullet \mathrm{mol}^{-1}\end{array}$ \\
\hline$\# 1$ & 0 & -35.0 & $\mathrm{~S}(13.62), \mathrm{B}(28.38), \mathrm{T}(82.73), \mathrm{IT}(4.53), \mathrm{VDW}(66.71), \mathrm{E}(-231.04)$ \\
\hline \#2 & 8.9 & -26.1 & $\mathrm{~S}(17.80), \mathrm{B}(44.41), \mathrm{T}(65.21), \mathrm{IT}(2.97), \mathrm{VDW}(78.29), \mathrm{E}(-234.83)$ \\
\hline
\end{tabular}


Table S6. Cartesian coordinates of the conformers of 1e

1e \#1

\begin{tabular}{lrrr}
\hline atom & $\mathrm{x}$ & $\mathrm{y}$ & $\mathrm{z}$ \\
\hline $\mathrm{C}$ & 6.29060 & 3.39040 & -0.58400 \\
$\mathrm{C}$ & 6.38140 & 3.19800 & -1.98160 \\
$\mathrm{C}$ & 6.56590 & 4.32620 & -2.83430 \\
$\mathrm{C}$ & 6.67410 & 5.61600 & -2.26100 \\
$\mathrm{C}$ & 6.58140 & 5.79080 & -0.86800 \\
$\mathrm{C}$ & 6.38700 & 4.67990 & -0.02890 \\
$\mathrm{H}$ & 6.16200 & 2.54310 & 0.07440 \\
$\mathrm{H}$ & 6.83340 & 6.48750 & -2.87920 \\
$\mathrm{H}$ & 6.66380 & 6.78160 & -0.44500 \\
$\mathrm{H}$ & 6.32180 & 4.81710 & 1.04120 \\
$\mathrm{C}$ & 6.28900 & 1.80200 & -2.53520 \\
$\mathrm{O}$ & 7.05500 & 1.41350 & -3.41630 \\
$\mathrm{H}$ & 4.60610 & 1.51370 & -1.47540 \\
$\mathrm{~N}$ & 5.25230 & 1.07000 & -2.10840 \\
$\mathrm{C}$ & 4.80440 & -0.14870 & -2.77970 \\
$\mathrm{C}$ & 4.10400 & 0.27560 & -4.08170 \\
$\mathrm{O}$ & 3.02360 & 0.86020 & -4.03680 \\
$\mathrm{H}$ & 5.70730 & -0.29750 & -5.15240 \\
$\mathrm{~N}$ & 4.76510 & 0.06170 & -5.22330 \\
$\mathrm{C}$ & 4.44410 & 0.70400 & -6.49600 \\
$\mathrm{H}$ & 3.41130 & 1.04920 & -6.51410 \\
\hline & & &
\end{tabular}

\begin{tabular}{lrrr}
\hline $\mathrm{C}$ & 5.47930 & 1.85190 & -6.61640 \\
$\mathrm{O}$ & 6.66560 & 1.53110 & -6.70470 \\
$\mathrm{~N}$ & 5.09470 & 3.15100 & -6.55410 \\
$\mathrm{C}$ & 6.10860 & 4.23510 & -6.61940 \\
$\mathrm{H}$ & 7.10880 & 3.84050 & -6.80530 \\
$\mathrm{C}$ & 3.69690 & 3.54040 & -6.30460 \\
$\mathrm{H}$ & 3.32300 & 3.09020 & -5.38440 \\
$\mathrm{H}$ & 3.56920 & 4.61630 & -6.18820 \\
$\mathrm{H}$ & 3.05560 & 3.23060 & -7.13090 \\
$\mathrm{C}$ & 6.20730 & 4.91570 & -5.24100 \\
$\mathrm{O}$ & 5.87360 & 6.09350 & -5.10500 \\
$\mathrm{~N}$ & 6.64650 & 4.12760 & -4.24330 \\
$\mathrm{H}$ & 6.91130 & 3.18710 & -4.52020 \\
$\mathrm{H}$ & 5.65500 & -0.79860 & -2.99610 \\
$\mathrm{C}$ & 4.63100 & -0.32730 & -7.64800 \\
$\mathrm{H}$ & 5.66850 & -0.66830 & -7.65470 \\
$\mathrm{C}$ & 3.74850 & -1.57590 & -7.44850 \\
$\mathrm{H}$ & 3.99940 & -2.10990 & -6.53190 \\
$\mathrm{H}$ & 2.69220 & -1.30960 & -7.39670 \\
$\mathrm{H}$ & 3.87250 & -2.28170 & -8.27040 \\
$\mathrm{C}$ & 4.34670 & 0.28340 & -9.03500 \\
$\mathrm{H}$ & 4.46220 & -0.45980 & -9.82440 \\
\hline
\end{tabular}

\begin{tabular}{lrrr}
\hline $\mathrm{H}$ & 5.03430 & 1.09690 & -9.26960 \\
$\mathrm{H}$ & 3.33240 & 0.67870 & -9.09860 \\
$\mathrm{C}$ & 5.82160 & 5.18660 & -7.82050 \\
$\mathrm{H}$ & 4.88380 & 5.71550 & -7.64720 \\
$\mathrm{C}$ & 5.45910 & 3.02750 & -11.57840 \\
$\mathrm{C}$ & 4.31910 & 3.60210 & -10.98410 \\
$\mathrm{C}$ & 4.43720 & 4.31030 & -9.77200 \\
$\mathrm{C}$ & 5.69600 & 4.44690 & -9.14710 \\
$\mathrm{C}$ & 6.83530 & 3.87290 & -9.75040 \\
$\mathrm{C}$ & 6.71760 & 3.16370 & -10.96160 \\
$\mathrm{H}$ & 5.37010 & 2.48630 & -12.50920 \\
$\mathrm{H}$ & 3.35450 & 3.50220 & -11.46030 \\
$\mathrm{H}$ & 3.55730 & 4.74900 & -9.32490 \\
$\mathrm{H}$ & 7.80220 & 3.98050 & -9.27970 \\
$\mathrm{H}$ & 7.59490 & 2.72790 & -11.41710 \\
$\mathrm{O}$ & 6.80860 & 6.19830 & -7.90320 \\
$\mathrm{H}$ & 6.74540 & 6.71830 & -7.11060 \\
$\mathrm{C}$ & 3.81300 & -0.88570 & -1.86250 \\
$\mathrm{H}$ & 4.29030 & -1.18870 & -0.93040 \\
$\mathrm{H}$ & 2.94990 & -0.26740 & -1.61060 \\
$\mathrm{H}$ & 3.43670 & -1.78900 & -2.34450 \\
\hline & & &
\end{tabular}


$1 \mathrm{e} \# 2$

\begin{tabular}{lrrr}
\hline atom & $\mathrm{x}$ & $\mathrm{y}$ & $\mathrm{z}$ \\
\hline $\mathrm{C}$ & 6.11090 & 3.37700 & -0.65060 \\
$\mathrm{C}$ & 6.25670 & 3.18990 & -2.04480 \\
$\mathrm{C}$ & 6.43660 & 4.32640 & -2.88880 \\
$\mathrm{C}$ & 6.47830 & 5.61770 & -2.30960 \\
$\mathrm{C}$ & 6.32660 & 5.78630 & -0.92130 \\
$\mathrm{C}$ & 6.14150 & 4.66760 & -0.09100 \\
$\mathrm{H}$ & 5.98820 & 2.52660 & 0.00480 \\
$\mathrm{H}$ & 6.63140 & 6.49740 & -2.91760 \\
$\mathrm{H}$ & 6.35710 & 6.77870 & -0.49500 \\
$\mathrm{H}$ & 6.03170 & 4.80060 & 0.97600 \\
$\mathrm{C}$ & 6.23140 & 1.79110 & -2.60040 \\
$\mathrm{O}$ & 7.00160 & 1.44510 & -3.49980 \\
$\mathrm{H}$ & 4.59430 & 1.40680 & -1.50430 \\
$\mathrm{~N}$ & 5.24370 & 1.00110 & -2.16000 \\
$\mathrm{C}$ & 4.90830 & -0.32510 & -2.69160 \\
$\mathrm{C}$ & 4.17740 & -0.19870 & -4.04930 \\
$\mathrm{O}$ & 3.03160 & -0.61390 & -4.19790 \\
$\mathrm{H}$ & 5.77240 & 0.75510 & -4.75140 \\
$\mathrm{~N}$ & 4.84890 & 0.43190 & -5.01760 \\
$\mathrm{C}$ & 4.35190 & 0.85600 & -6.32480 \\
$\mathrm{H}$ & 3.26040 & 0.83590 & -6.27220 \\
\hline & & &
\end{tabular}

\begin{tabular}{lrrr}
\hline $\mathrm{C}$ & 4.67590 & 2.36090 & -6.51680 \\
$\mathrm{O}$ & 3.73390 & 3.14340 & -6.42050 \\
$\mathrm{~N}$ & 5.95210 & 2.81490 & -6.67610 \\
$\mathrm{C}$ & 6.25790 & 4.27450 & -6.73170 \\
$\mathrm{H}$ & 7.30220 & 4.38070 & -7.02480 \\
$\mathrm{C}$ & 7.11140 & 1.92310 & -6.83660 \\
$\mathrm{H}$ & 7.08430 & 1.05550 & -6.18090 \\
$\mathrm{H}$ & 7.16870 & 1.57400 & -7.86850 \\
$\mathrm{H}$ & 8.05530 & 2.42130 & -6.61420 \\
$\mathrm{C}$ & 6.21040 & 4.91520 & -5.31960 \\
$\mathrm{O}$ & 5.85070 & 6.08530 & -5.18590 \\
$\mathrm{~N}$ & 6.59140 & 4.13210 & -4.29270 \\
$\mathrm{H}$ & 6.88490 & 3.19930 & -4.55090 \\
$\mathrm{H}$ & 5.82080 & -0.90520 & -2.84200 \\
$\mathrm{C}$ & 4.71840 & -0.15300 & -7.45840 \\
$\mathrm{H}$ & 5.79110 & -0.33680 & -7.43930 \\
$\mathrm{C}$ & 4.05310 & -1.52650 & -7.24770 \\
$\mathrm{H}$ & 4.38120 & -1.99610 & -6.32080 \\
$\mathrm{H}$ & 2.96660 & -1.43910 & -7.20400 \\
$\mathrm{H}$ & 4.29780 & -2.21620 & -8.05580 \\
$\mathrm{C}$ & 4.38230 & 0.36870 & -8.86650 \\
$\mathrm{H}$ & 4.58580 & -0.38350 & -9.62920 \\
\hline & & &
\end{tabular}

\begin{tabular}{lrrr}
\hline $\mathrm{H}$ & 4.98210 & 1.24110 & -9.12300 \\
$\mathrm{H}$ & 3.33110 & 0.64800 & -8.94940 \\
$\mathrm{C}$ & 5.46940 & 5.05060 & -7.84260 \\
$\mathrm{H}$ & 4.43610 & 5.20030 & -7.52760 \\
$\mathrm{C}$ & 5.44050 & 3.07010 & -11.71410 \\
$\mathrm{C}$ & 4.23530 & 3.26330 & -11.01170 \\
$\mathrm{C}$ & 4.24600 & 3.90460 & -9.75740 \\
$\mathrm{C}$ & 5.46100 & 4.35780 & -9.20010 \\
$\mathrm{C}$ & 6.66390 & 4.17370 & -9.91510 \\
$\mathrm{C}$ & 6.65470 & 3.52740 & -11.16660 \\
$\mathrm{H}$ & 5.43190 & 2.58100 & -12.67740 \\
$\mathrm{H}$ & 3.30180 & 2.92000 & -11.43360 \\
$\mathrm{H}$ & 3.31800 & 4.04720 & -9.22150 \\
$\mathrm{H}$ & 7.59250 & 4.54350 & -9.50540 \\
$\mathrm{H}$ & 7.57790 & 3.39380 & -11.71160 \\
$\mathrm{O}$ & 6.00000 & 6.35660 & -7.97620 \\
$\mathrm{H}$ & 5.88770 & 6.78380 & -7.13230 \\
$\mathrm{C}$ & 4.01900 & -1.04330 & -1.65860 \\
$\mathrm{H}$ & 4.53140 & -1.15480 & -0.70300 \\
$\mathrm{H}$ & 3.08660 & -0.50490 & -1.48070 \\
$\mathrm{H}$ & 3.75450 & -2.04470 & -2.00190 \\
\hline & & &
\end{tabular}


3D structures, calculated total energies, relative energies and the atomic coordinates of $\mathbf{1 f}$.

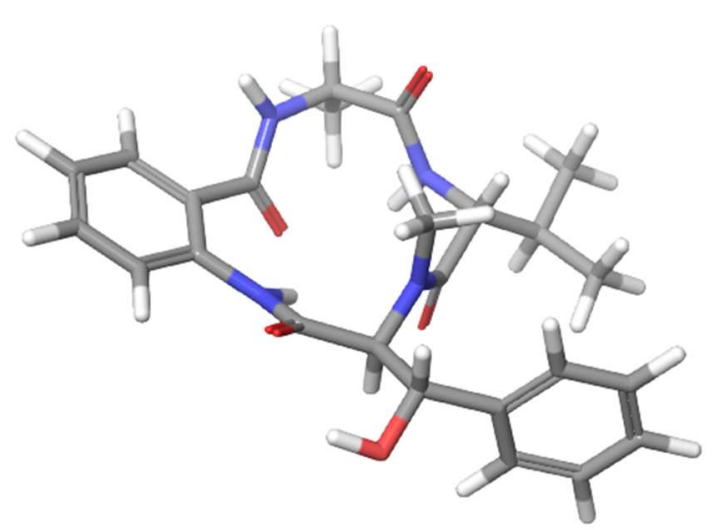

$\# 1$

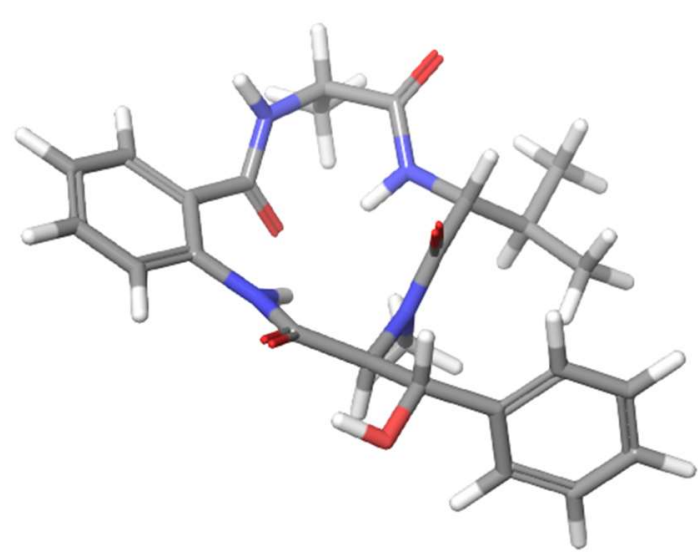

\#2

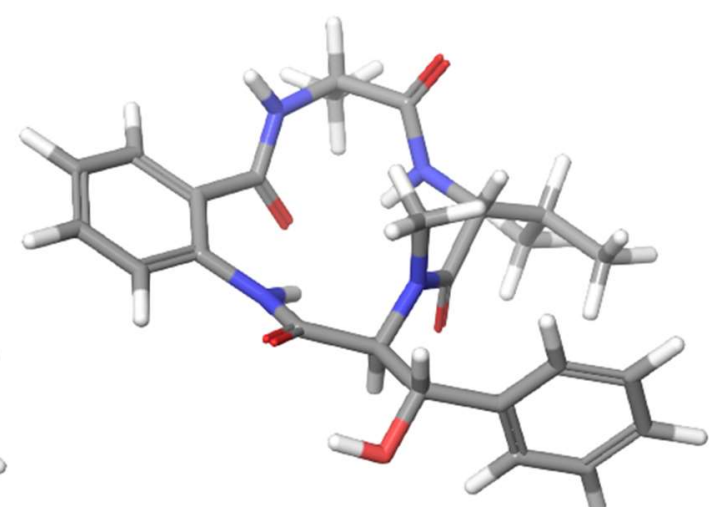

\#3

Figure S3. 3D structures of the conformers of $\mathbf{1 f}$

Table S7. Total and relative energies for conformers of $1 \mathrm{f}$

\begin{tabular}{|c|c|c|c|}
\hline No. & $\begin{array}{c}\text { Relative Energy } \\
/ \mathrm{kJ} \mathrm{mol}^{-1}\end{array}$ & $\begin{array}{c}\text { Total Energy } \\
/ \mathrm{kJ}^{\prime} \mathrm{mol}^{-1}\end{array}$ & $\begin{array}{c}\text { Stretch (S), Bend (B), Torsional (T), Improper Torsional (IT), } \\
\text { Van der Waals (VDW), and Electrostatic (E) Energies } / \mathrm{kJ}^{-} \mathrm{mol}^{-1}\end{array}$ \\
\hline$\# 1$ & 0 & -17.4 & $\mathrm{~S}(13.50), \mathrm{B}(33.40), \mathrm{T}(95.80), \mathrm{IT}(4.13), \mathrm{VDW}(63.88), \mathrm{E}(-228.08)$ \\
\hline$\# 2$ & 1.9 & -15.5 & $\mathrm{~S}(17.63), \mathrm{B}(50.63), \mathrm{T}(70.86), \mathrm{IT}(3.46), \mathrm{VDW}(74.43), \mathrm{E}(-232.46)$ \\
\hline$\# 3$ & 7.9 & -9.5 & $\mathrm{~S}(14.16), \mathrm{B}(39.43), \mathrm{T}(89.01), \mathrm{IT}(3.94), \mathrm{VDW}(64.53), \mathrm{E}(-220.52)$ \\
\hline
\end{tabular}




\section{Table S8. Cartesian coordinates of the conformers of 1f}

\section{$1 \mathrm{f} \# \mathbf{1}$}

\begin{tabular}{lrrr}
\hline atom & $\mathrm{x}$ & $\mathrm{y}$ & $\mathrm{z}$ \\
\hline $\mathrm{C}$ & -5.18910 & 1.32000 & -0.81620 \\
$\mathrm{C}$ & -5.65880 & 1.42860 & -2.14520 \\
$\mathrm{C}$ & -6.52170 & 0.42040 & -2.66680 \\
$\mathrm{C}$ & -6.90430 & -0.66190 & -1.83950 \\
$\mathrm{C}$ & -6.42710 & -0.75620 & -0.51930 \\
$\mathrm{C}$ & -5.56850 & 0.23250 & -0.00730 \\
$\mathrm{H}$ & -4.54490 & 2.08260 & -0.40250 \\
$\mathrm{H}$ & -7.57000 & -1.43030 & -2.20590 \\
$\mathrm{H}$ & -6.72590 & -1.58880 & 0.10110 \\
$\mathrm{H}$ & -5.20910 & 0.16020 & 1.00950 \\
$\mathrm{C}$ & -5.23840 & 2.61000 & -2.97700 \\
$\mathrm{O}$ & -6.05270 & 3.21910 & -3.66940 \\
$\mathrm{H}$ & -3.34100 & 2.22110 & -2.47920 \\
$\mathrm{~N}$ & -3.92040 & 2.85050 & -3.01240 \\
$\mathrm{C}$ & -3.21710 & 3.77090 & -3.91220 \\
$\mathrm{H}$ & -2.17090 & 3.74780 & -3.60370 \\
$\mathrm{C}$ & -3.24640 & 3.21880 & -5.34580 \\
$\mathrm{O}$ & -2.33490 & 2.49880 & -5.74240 \\
$\mathrm{H}$ & -5.08150 & 3.98600 & -5.61380 \\
$\mathrm{~N}$ & -4.33140 & 3.48810 & -6.07870 \\
$\mathrm{C}$ & -4.70290 & 2.82600 & -7.32510 \\
\hline & & &
\end{tabular}

\begin{tabular}{lrrr}
\hline $\mathrm{H}$ & -3.92050 & 2.14620 & -7.65990 \\
$\mathrm{C}$ & -6.01440 & 2.07910 & -6.98250 \\
$\mathrm{O}$ & -7.03320 & 2.75720 & -6.84790 \\
$\mathrm{~N}$ & -6.01230 & 0.74260 & -6.75200 \\
$\mathrm{C}$ & -7.26910 & 0.04360 & -6.37880 \\
$\mathrm{H}$ & -8.12200 & 0.72430 & -6.37810 \\
$\mathrm{C}$ & -4.77070 & -0.04970 & -6.75840 \\
$\mathrm{H}$ & -4.33860 & -0.08300 & -7.75940 \\
$\mathrm{H}$ & -4.03210 & 0.37060 & -6.07460 \\
$\mathrm{H}$ & -4.92490 & -1.08270 & -6.44670 \\
$\mathrm{C}$ & -7.16730 & -0.44150 & -4.92040 \\
$\mathrm{O}$ & -7.22140 & -1.64360 & -4.65780 \\
$\mathrm{~N}$ & -6.98970 & 0.52940 & -4.00700 \\
$\mathrm{H}$ & -6.99660 & 1.47540 & -4.37510 \\
$\mathrm{C}$ & -3.68790 & 5.23610 & -3.78330 \\
$\mathrm{H}$ & -4.73180 & 5.37490 & -4.06450 \\
$\mathrm{H}$ & -3.57320 & 5.59400 & -2.76020 \\
$\mathrm{H}$ & -3.09330 & 5.88820 & -4.42420 \\
$\mathrm{C}$ & -4.90140 & 3.89140 & -8.44280 \\
$\mathrm{H}$ & -5.70100 & 4.57120 & -8.14150 \\
$\mathrm{C}$ & -3.63770 & 4.74880 & -8.65470 \\
$\mathrm{H}$ & -3.37730 & 5.31530 & -7.76050 \\
\hline
\end{tabular}

\begin{tabular}{lrrr}
\hline $\mathrm{H}$ & -2.77840 & 4.12970 & -8.91470 \\
$\mathrm{H}$ & -3.78150 & 5.47220 & -9.45770 \\
$\mathrm{C}$ & -5.32430 & 3.25880 & -9.78400 \\
$\mathrm{H}$ & -5.44930 & 4.01870 & -10.55580 \\
$\mathrm{H}$ & -6.27730 & 2.73390 & -9.70350 \\
$\mathrm{H}$ & -4.58250 & 2.54370 & -10.14070 \\
$\mathrm{C}$ & -7.62390 & -1.04400 & -7.43690 \\
$\mathrm{H}$ & -6.86630 & -1.82830 & -7.41240 \\
$\mathrm{C}$ & -7.72490 & 0.59890 & -11.46240 \\
$\mathrm{C}$ & -6.67330 & -0.25450 & -11.07690 \\
$\mathrm{C}$ & -6.64770 & -0.79920 & -9.77800 \\
$\mathrm{C}$ & -7.67240 & -0.49140 & -8.85660 \\
$\mathrm{C}$ & -8.72680 & 0.35940 & -9.25140 \\
$\mathrm{C}$ & -8.75270 & 0.90450 & -10.54980 \\
$\mathrm{H}$ & -7.74660 & 1.01570 & -12.45890 \\
$\mathrm{H}$ & -5.88790 & -0.49330 & -11.77920 \\
$\mathrm{H}$ & -5.83680 & -1.45280 & -9.49190 \\
$\mathrm{H}$ & -9.51640 & 0.59400 & -8.55170 \\
$\mathrm{H}$ & -9.56300 & 1.55580 & -10.84360 \\
$\mathrm{O}$ & -8.84090 & -1.68720 & -7.10600 \\
$\mathrm{H}$ & -8.71140 & -2.12250 & -6.27130 \\
\hline
\end{tabular}




\section{$1 f$ \#2}

\begin{tabular}{lrrr}
\hline atom & $\mathrm{x}$ & $\mathrm{y}$ & $\mathrm{z}$ \\
\hline $\mathrm{C}$ & -5.13820 & 1.29080 & -0.84410 \\
$\mathrm{C}$ & -5.63500 & 1.43520 & -2.16000 \\
$\mathrm{C}$ & -6.51390 & 0.44390 & -2.69000 \\
$\mathrm{C}$ & -6.88100 & -0.65850 & -1.88140 \\
$\mathrm{C}$ & -6.37440 & -0.78930 & -0.57560 \\
$\mathrm{C}$ & -5.50260 & 0.18340 & -0.05620 \\
$\mathrm{H}$ & -4.48240 & 2.03930 & -0.42270 \\
$\mathrm{H}$ & -7.55820 & -1.41670 & -2.24730 \\
$\mathrm{H}$ & -6.66110 & -1.63820 & 0.02840 \\
$\mathrm{H}$ & -5.12050 & 0.08230 & 0.94990 \\
$\mathrm{C}$ & -5.22770 & 2.63730 & -2.96850 \\
$\mathrm{O}$ & -6.04260 & 3.23120 & -3.67790 \\
$\mathrm{H}$ & -3.33150 & 2.27820 & -2.45740 \\
$\mathrm{~N}$ & -3.91460 & 2.90580 & -2.99000 \\
$\mathrm{C}$ & -3.20020 & 3.89480 & -3.81460 \\
$\mathrm{H}$ & -2.17450 & 3.89610 & -3.44250 \\
$\mathrm{C}$ & -3.11460 & 3.46770 & -5.29070 \\
$\mathrm{O}$ & -2.02820 & 3.41110 & -5.85620 \\
$\mathrm{H}$ & -5.08260 & 3.21140 & -5.27930 \\
$\mathrm{~N}$ & -4.26580 & 3.11910 & -5.87350 \\
$\mathrm{C}$ & -4.45960 & 2.47520 & -7.17010 \\
\hline & & &
\end{tabular}

\begin{tabular}{lrrr}
\hline $\mathrm{H}$ & -3.49950 & 2.03180 & -7.44620 \\
$\mathrm{C}$ & -5.38230 & 1.24360 & -6.97390 \\
$\mathrm{O}$ & -4.83970 & 0.14150 & -6.99080 \\
$\mathrm{~N}$ & -6.70990 & 1.36820 & -6.68690 \\
$\mathrm{C}$ & -7.55730 & 0.17790 & -6.38300 \\
$\mathrm{H}$ & -8.59460 & 0.51190 & -6.36440 \\
$\mathrm{C}$ & -7.41390 & 2.66000 & -6.67370 \\
$\mathrm{H}$ & -8.34180 & 2.62360 & -6.10250 \\
$\mathrm{H}$ & -6.83310 & 3.46340 & -6.22540 \\
$\mathrm{H}$ & -7.67560 & 2.94950 & -7.69230 \\
$\mathrm{C}$ & -7.31520 & -0.33360 & -4.93850 \\
$\mathrm{O}$ & -7.41110 & -1.53480 & -4.68520 \\
$\mathrm{~N}$ & -7.01840 & 0.59910 & -4.01430 \\
$\mathrm{H}$ & -6.99100 & 1.55000 & -4.35810 \\
$\mathrm{C}$ & -3.74180 & 5.32700 & -3.63960 \\
$\mathrm{H}$ & -3.74960 & 5.62160 & -2.59020 \\
$\mathrm{H}$ & -3.11330 & 6.04090 & -4.17380 \\
$\mathrm{H}$ & -4.75400 & 5.44030 & -4.02800 \\
$\mathrm{C}$ & -4.77220 & 3.49430 & -8.31160 \\
$\mathrm{H}$ & -5.61490 & 4.11600 & -8.01540 \\
$\mathrm{C}$ & -3.60360 & 4.46700 & -8.55910 \\
$\mathrm{H}$ & -3.39490 & 5.07800 & -7.68130 \\
\hline & & &
\end{tabular}

\begin{tabular}{lrrr}
\hline $\mathrm{H}$ & -2.68730 & 3.93190 & -8.81190 \\
$\mathrm{H}$ & -3.82280 & 5.15380 & -9.37690 \\
$\mathrm{C}$ & -5.16340 & 2.81580 & -9.63640 \\
$\mathrm{H}$ & -5.31400 & 3.54860 & -10.42970 \\
$\mathrm{H}$ & -6.09730 & 2.26280 & -9.54290 \\
$\mathrm{H}$ & -4.39370 & 2.11930 & -9.97140 \\
$\mathrm{C}$ & -7.53470 & -0.92590 & -7.49620 \\
$\mathrm{H}$ & -6.59880 & -1.48340 & -7.44680 \\
$\mathrm{C}$ & -7.99170 & 0.61920 & -11.53560 \\
$\mathrm{C}$ & -6.79430 & -0.01700 & -11.15510 \\
$\mathrm{C}$ & -6.64740 & -0.51690 & -9.84630 \\
$\mathrm{C}$ & -7.69710 & -0.38520 & -8.91190 \\
$\mathrm{C}$ & -8.89960 & 0.24170 & -9.30310 \\
$\mathrm{C}$ & -9.04530 & 0.74660 & -10.61000 \\
$\mathrm{H}$ & -8.10550 & 0.99970 & -12.54030 \\
$\mathrm{H}$ & -5.98770 & -0.12240 & -11.86610 \\
$\mathrm{H}$ & -5.72470 & -1.00160 & -9.55930 \\
$\mathrm{H}$ & -9.71590 & 0.32070 & -8.59980 \\
$\mathrm{H}$ & -9.97000 & 1.22060 & -10.90530 \\
$\mathrm{O}$ & -8.54170 & -1.88590 & -7.23260 \\
$\mathrm{H}$ & -8.33840 & -2.26840 & -6.38430 \\
\hline & & & \\
\hline
\end{tabular}




\section{1f \#3}

\begin{tabular}{lrrr}
\hline atom & $\mathrm{x}$ & $\mathrm{y}$ & $\mathrm{z}$ \\
\hline $\mathrm{C}$ & -5.12880 & 1.29900 & -0.85430 \\
$\mathrm{C}$ & -5.61520 & 1.42010 & -2.17630 \\
$\mathrm{C}$ & -6.54990 & 0.46120 & -2.66810 \\
$\mathrm{C}$ & -6.98470 & -0.58310 & -1.81790 \\
$\mathrm{C}$ & -6.48970 & -0.69000 & -0.50530 \\
$\mathrm{C}$ & -5.56070 & 0.24860 & -0.02320 \\
$\mathrm{H}$ & -4.43150 & 2.02530 & -0.46160 \\
$\mathrm{H}$ & -7.70500 & -1.31250 & -2.15980 \\
$\mathrm{H}$ & -6.82950 & -1.49320 & 0.13260 \\
$\mathrm{H}$ & -5.18810 & 0.16740 & 0.98820 \\
$\mathrm{C}$ & -5.14070 & 2.56700 & -3.02780 \\
$\mathrm{O}$ & -5.93120 & 3.21280 & -3.71570 \\
$\mathrm{H}$ & -3.26140 & 2.08790 & -2.54350 \\
$\mathrm{~N}$ & -3.81160 & 2.73930 & -3.08140 \\
$\mathrm{C}$ & -3.05630 & 3.66260 & -3.94120 \\
$\mathrm{H}$ & -2.01540 & 3.57680 & -3.62560 \\
$\mathrm{C}$ & -3.09530 & 3.20960 & -5.41010 \\
$\mathrm{O}$ & -2.10090 & 2.72410 & -5.93940 \\
$\mathrm{H}$ & -5.05040 & 3.64710 & -5.46720 \\
$\mathrm{~N}$ & -4.27680 & 3.29500 & -6.02380 \\
$\mathrm{C}$ & -4.64440 & 2.73360 & -7.31560 \\
\hline & & &
\end{tabular}

\begin{tabular}{lrrr}
\hline $\mathrm{H}$ & -3.89930 & 1.99600 & -7.60900 \\
$\mathrm{C}$ & -5.99950 & 2.02250 & -7.08510 \\
$\mathrm{O}$ & -7.02300 & 2.70200 & -7.12870 \\
$\mathrm{~N}$ & -6.03070 & 0.71460 & -6.72050 \\
$\mathrm{C}$ & -7.31710 & 0.05850 & -6.36970 \\
$\mathrm{H}$ & -8.14620 & 0.76720 & -6.40100 \\
$\mathrm{C}$ & -4.80600 & -0.09540 & -6.58820 \\
$\mathrm{H}$ & -4.30450 & -0.19670 & -7.55170 \\
$\mathrm{H}$ & -4.10990 & 0.35360 & -5.87810 \\
$\mathrm{H}$ & -5.00210 & -1.10880 & -6.23730 \\
$\mathrm{C}$ & -7.28160 & -0.39540 & -4.89710 \\
$\mathrm{O}$ & -7.44000 & -1.58180 & -4.60800 \\
$\mathrm{~N}$ & -7.03260 & 0.57840 & -4.00280 \\
$\mathrm{H}$ & -6.97670 & 1.51450 & -4.38890 \\
$\mathrm{C}$ & -3.46360 & 5.13770 & -3.74580 \\
$\mathrm{H}$ & -4.49170 & 5.33470 & -4.05050 \\
$\mathrm{H}$ & -3.36300 & 5.43870 & -2.70310 \\
$\mathrm{H}$ & -2.82370 & 5.79380 & -4.33730 \\
$\mathrm{C}$ & -4.57310 & 3.81950 & -8.42990 \\
$\mathrm{H}$ & -3.51800 & 4.08220 & -8.52440 \\
$\mathrm{C}$ & -5.00340 & 3.27450 & -9.80450 \\
$\mathrm{H}$ & -4.45040 & 2.37150 & -10.06480 \\
\hline
\end{tabular}

\begin{tabular}{lrrr}
\hline $\mathrm{H}$ & -6.06640 & 3.02830 & -9.82620 \\
$\mathrm{H}$ & -4.82240 & 4.00640 & -10.59210 \\
$\mathrm{C}$ & -5.30960 & 5.13790 & -8.10920 \\
$\mathrm{H}$ & -5.14400 & 5.87450 & -8.89570 \\
$\mathrm{H}$ & -4.96010 & 5.58410 & -7.17830 \\
$\mathrm{H}$ & -6.38680 & 4.99520 & -8.02580 \\
$\mathrm{C}$ & -7.67590 & -1.03170 & -7.42320 \\
$\mathrm{H}$ & -6.94590 & -1.83950 & -7.35450 \\
$\mathrm{C}$ & -7.54960 & 0.54470 & -11.47390 \\
$\mathrm{C}$ & -8.60640 & 0.89690 & -10.61260 \\
$\mathrm{C}$ & -8.65650 & 0.37060 & -9.30730 \\
$\mathrm{C}$ & -7.64880 & -0.50700 & -8.85380 \\
$\mathrm{C}$ & -6.59520 & -0.86240 & -9.72420 \\
$\mathrm{C}$ & -6.54530 & -0.33700 & -11.03030 \\
$\mathrm{H}$ & -7.51290 & 0.94790 & -12.47550 \\
$\mathrm{H}$ & -9.37990 & 1.57120 & -10.95050 \\
$\mathrm{H}$ & -9.46600 & 0.64430 & -8.64560 \\
$\mathrm{H}$ & -5.81880 & -1.53590 & -9.39260 \\
$\mathrm{H}$ & -5.73770 & -0.61120 & -11.69350 \\
$\mathrm{O}$ & -8.92630 & -1.62530 & -7.12630 \\
$\mathrm{H}$ & -8.84670 & -2.03990 & -6.27480 \\
\hline & & & \\
\hline & & &
\end{tabular}


Table S9. Comparison of ${ }^{3} J_{\mathrm{H}, \mathrm{H}}$ coupling constants in the natural 1 , synthetic 1a, and model tetrapeptide $1 \mathrm{~d}$

\begin{tabular}{|c|c|c|c|}
\hline \multirow[b]{2}{*}{ Vicinal protons } & \multicolumn{3}{|c|}{$J_{\mathrm{H}, \mathrm{H}}$ coupling constants $(\mathrm{Hz})$} \\
\hline & $\begin{array}{l}\text { Natural 1 }{ }^{\mathrm{a}} \\
\text { (observed) }\end{array}$ & $\begin{array}{c}\text { Synthetic 1a } \\
\text { (observed) }\end{array}$ & $\begin{array}{c}\mathbf{1 d}^{\mathrm{b}} \\
\text { (theoretical) }\end{array}$ \\
\hline MePhe(3-OH) $\mathrm{H}_{\alpha} /$ MePhe(3-OH) $\mathrm{H}_{\beta}$ & 10.0 & 2.6 & 2.0 \\
\hline Ile $\mathrm{H}_{\alpha} /$ Ile $\mathrm{H}_{\beta}\left(\right.$ Val $\left.\mathrm{H}_{\alpha} / \mathrm{Val} \mathrm{H}_{\beta}\right)$ & $10.0^{\mathrm{c}}$ & $9.5^{\mathrm{c}}$ & $10.7^{\mathrm{d}}$ \\
\hline Ile NH / Ile $\mathrm{H}_{\alpha}\left(\right.$ Val NH / Val H $\left.{ }_{\alpha}\right)$ & $9.5^{\mathrm{c}}$ & $9.5^{\mathrm{c}}$ & $9.5^{\mathrm{d}}$ \\
\hline Ala NH / Ala $\mathrm{H}_{\alpha}$ & 8.0 & 3.8 & 6.5 \\
\hline
\end{tabular}

a Observed values in the ${ }^{1} \mathrm{H}$ NMR spectra of the natural $\mathbf{1}$ and synthetic 1a. ${ }^{\mathrm{b}}$ Calculated values in the lowest-energy structure of model tetrapeptide $\mathbf{1 d} .{ }^{\mathrm{c}} \mathrm{J}_{\mathrm{H}, \mathrm{H}}$ coupling constants observed between protons of the Ile residue. ${ }^{d 3} J_{\mathrm{H}, \mathrm{H}}$ coupling constants calculated between protons of the Val residue.

Table S10. Comparison of distances between proximal protons in the natural 1, synthetic 1a, and model tetrapeptide 1d

\begin{tabular}{|c|c|c|c|}
\hline & \multicolumn{2}{|c|}{$\begin{array}{c}\text { NOE correlation } \\
\text { observation }^{\mathrm{a}}\end{array}$} & \multirow{2}{*}{$\begin{array}{c}\text { Theoretical } \\
\text { distance }^{\mathrm{b}}(\AA)\end{array}$} \\
\hline Proximal protons & Natural 1 & Synthetic 1a & \\
\hline MePhe(3-OH) $\mathrm{H}_{\alpha} /$ Ant NH & $\mathrm{Y}$ & $\mathrm{Y}$ & 3.55 \\
\hline MePhe(3-OH) $\mathrm{H}_{\beta} / \mathrm{MePhe}(3-\mathrm{OH}) \mathrm{NMe}$ & $\mathrm{Y}$ & $\mathrm{N}$ & 4.36 \\
\hline MePhe(3-OH) NMe / Ile $\mathrm{H}_{\alpha}\left(\mathrm{MePhe}(3-\mathrm{OH}) \mathrm{NMe} / \mathrm{Val} \mathrm{H}{ }_{\alpha}\right)$ & $\mathrm{Y}^{\mathrm{c}}$ & $\mathrm{Y}^{\mathrm{c}}$ & $2.32^{\mathrm{d}}$ \\
\hline Ile NH / Ala $\mathrm{H}_{\alpha}\left(\right.$ Val NH / Ala $\left.\mathrm{H}_{\alpha}\right)$ & $\mathrm{Y}^{\mathrm{c}}$ & $\mathrm{N}$ & $3.55^{\mathrm{d}}$ \\
\hline Ala NH / Ala $\mathrm{H}_{\beta}$ & $\mathrm{Y}$ & $\mathrm{Y}$ & 3.39 \\
\hline
\end{tabular}

${ }^{a}$ NOE correlations observed in the natural 1 and synthetic 1a. Y: observed, N: not observed. ${ }^{b}$ Calculated distances in the model tetrapeptide 1d. ${ }^{c}$ NOE observation in protons of the Ile residue. ${ }^{\mathrm{d}}$ Theoretical distance in protons of the Val residue. 
Table S11. Comparison of ${ }^{3} J_{\mathrm{H}, \mathrm{H}}$ coupling constants in the natural 1 and model tetrapeptides $1 \mathrm{e}$ and $1 \mathrm{f}$

\begin{tabular}{|c|c|c|c|}
\hline & \multicolumn{3}{|c|}{$J_{\mathrm{H}, \mathrm{H}}$ coupling constants $(\mathrm{Hz})$} \\
\hline Vicinal protons & $\begin{array}{c}\text { Natural } \mathbf{1}^{\mathrm{a}} \\
\text { (observed) }\end{array}$ & $\begin{array}{c}\mathbf{1 e}^{\mathrm{b}} \\
\text { (theoretical) }\end{array}$ & $\begin{array}{c}\mathbf{1 f}^{\mathrm{b}} \\
\text { (theoretical) }\end{array}$ \\
\hline $\mathrm{MePhe}(3-\mathrm{OH}) \mathrm{H}_{\alpha} / \mathrm{MePhe}(3-\mathrm{OH}) \mathrm{H}_{\beta}$ & 10.0 & 9.5 & 9.5 \\
\hline $\mathrm{Ile} \mathrm{H}_{\alpha} / \mathrm{Ile}_{\beta}\left(\mathrm{Val} \mathrm{H} / \mathrm{H}_{\alpha} / \mathrm{Val} \mathrm{H}_{\beta}\right)$ & $10.0^{\mathrm{c}}$ & $10.7^{\mathrm{d}}$ & $10.7^{\mathrm{d}}$ \\
\hline $\mathrm{Ile} \mathrm{NH} / \mathrm{Ile} \mathrm{H}_{\alpha}\left(\mathrm{Val} \mathrm{NH} / \mathrm{Val} \mathrm{H}_{\alpha}\right)$ & $9.5^{\mathrm{c}}$ & $9.5^{\mathrm{d}}$ & $9.2^{\mathrm{d}}$ \\
\hline Ala NH / Ala H $\mathrm{H}_{\alpha}$ & 8.0 & 7.7 & 6.5 \\
\hline
\end{tabular}

${ }^{a}$ Observed values in the ${ }^{1} \mathrm{H}$ NMR spectra of the natural 1. ${ }^{\mathrm{b}}$ Calculated values in the lowest-energy structures of model tetrapeptides $1 \mathbf{e}$ and $\mathbf{1 f} .{ }^{\mathrm{c}} J_{\mathrm{H}, \mathrm{H}}$ coupling constants observed between protons of the Ile residue. ${ }^{d 3}{ }_{\mathrm{H}} \mathrm{H}$ coupling constants calculated between protons of the Val residue.

\section{Table S12. Comparison of distances between proximal protons in the natural 1 and model tetrapeptides $1 \mathrm{e}$ and $1 \mathrm{f}$}

\begin{tabular}{|c|c|c|c|}
\hline & $\begin{array}{c}\text { NOE correlation } \\
\text { observation }\end{array}$ & \multicolumn{2}{|c|}{ Theoretical distances $^{\mathrm{b}}(\AA)$} \\
\hline Proximal protons & Natural 1 & $\mathbf{1 e}$ & $\mathbf{1 f}$ \\
\hline $\mathrm{MePhe}(3-\mathrm{OH}) \mathrm{H}_{\alpha} / \mathrm{Ant} \mathrm{NH}$ & $\mathrm{Y}$ & 2.38 & 2.42 \\
\hline $\mathrm{MePhe}(3-\mathrm{OH}) \mathrm{H}_{\beta} / \mathrm{MePhe}(3-\mathrm{OH}) \mathrm{NMe}$ & $\mathrm{Y}$ & 2.84 & 2.29 \\
\hline $\mathrm{MePhe}(3-\mathrm{OH}) \mathrm{NMe} / \mathrm{Ile} \mathrm{H}_{\alpha}\left(\mathrm{MePhe}(3-\mathrm{OH}) \mathrm{NMe} / \mathrm{Val} \mathrm{H}_{\alpha}\right)$ & $\mathrm{Y}^{\mathrm{c}}$ & $2.29^{\mathrm{d}}$ & $2.27^{\mathrm{d}}$ \\
\hline $\mathrm{Ile} \mathrm{NH} / \mathrm{Ala} \mathrm{H}_{\alpha}\left(\mathrm{Val} \mathrm{NH} / \mathrm{Ala} \mathrm{H}_{\alpha}\right)$ & $\mathrm{Y}^{\mathrm{c}}$ & $2.21^{\mathrm{d}}$ & $3.55^{\mathrm{d}}$ \\
\hline Ala NH $/ \mathrm{Ala}_{\beta}$ & $\mathrm{Y}$ & 2.44 & 3.39 \\
\hline
\end{tabular}

${ }^{a}$ NOE correlations observed in the natural 1. Y: observed. ${ }^{b}$ Calculated distances in the model tetrapeptides 1e and 1f. ${ }^{\mathrm{c}}$ NOE observation in protons of the Ile residue. ${ }^{\mathrm{d}}$ Theoretical distance in protons of the Val residue. 
Table S13. A panel of three cancer cell lines for cytotoxicity

\begin{tabular}{|c|c|c|c|c|}
\hline Cancer cell line & Tissue & Resource $^{\text {a }}$ (resource No.) & Culture medium $^{\mathrm{b}}$ & $\begin{array}{c}\text { Number of cells } \\
\text { (cells/well) }\end{array}$ \\
\hline U937 & Blood & JCRB (JCRB9021) & $\begin{array}{c}\text { RPMI-1640 } \\
10 \% \text { FBS }\end{array}$ & 500 \\
\hline MOLT4 & Blood & JCRB (JCRB9031) & RPMI-1640 & 500 \\
\hline A549 & Lung & JCRB (JCRB0076) & EMEM, 10\% FBS & 500 \\
\hline
\end{tabular}

a JCRB: Japanese Collection of Research Bioresources. ${ }^{\mathrm{b}}$ All the media include $100 \mathrm{unit} / \mathrm{mL}$ penicillin and $100 \mu \mathrm{g} / \mathrm{mL}$ streptomycin.

U937
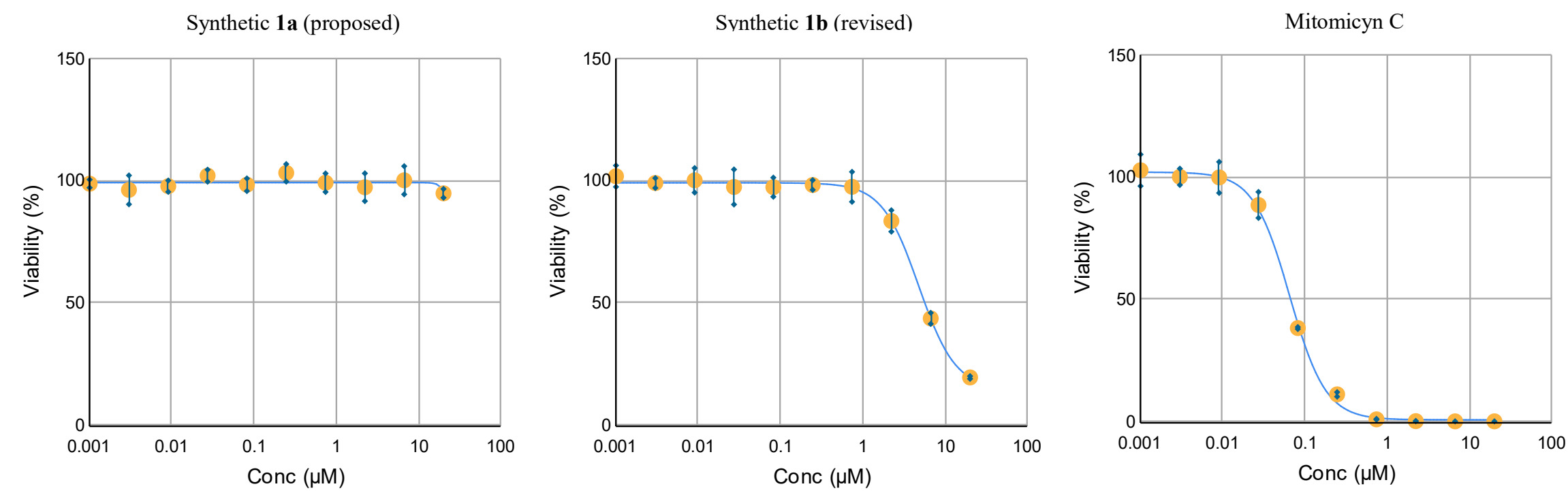
MOLT4

Synthetic 1a (proposed)

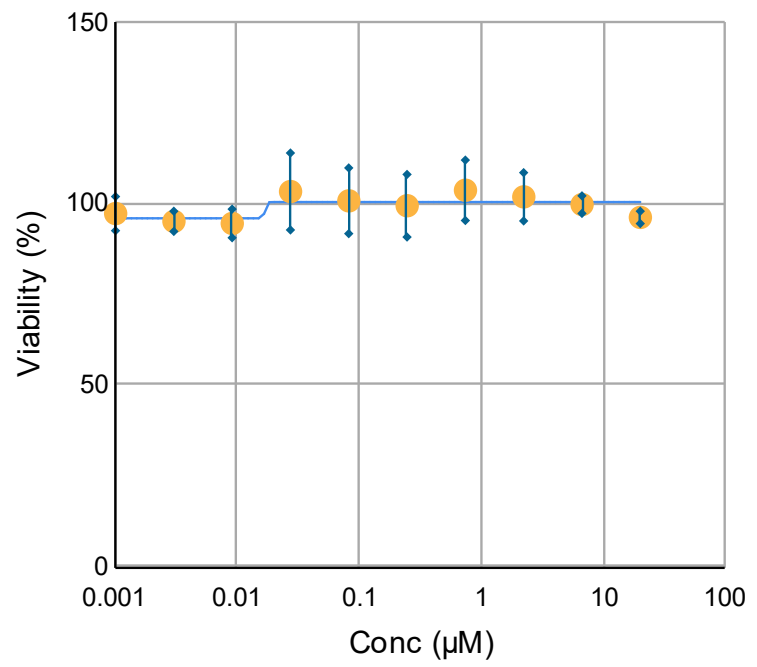

Synthetic 1b (revised)

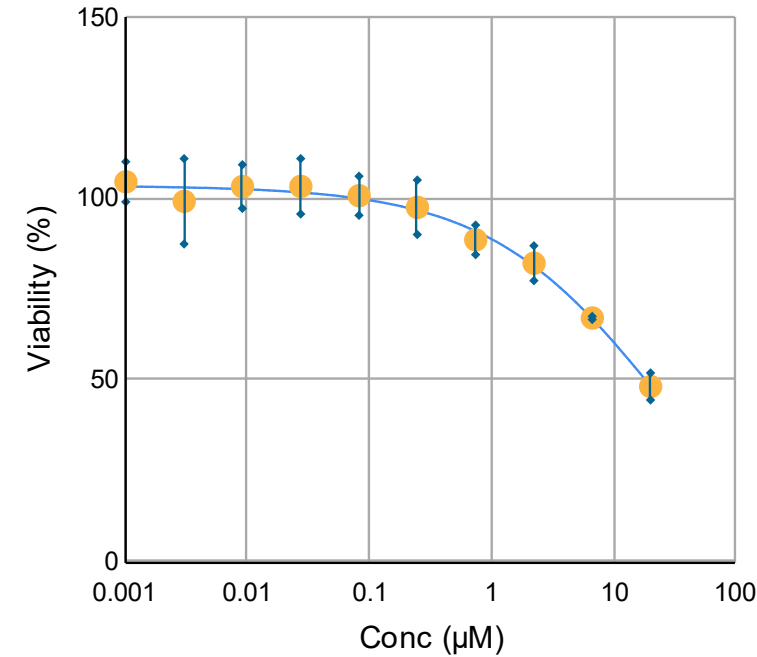

Mitomicyn C

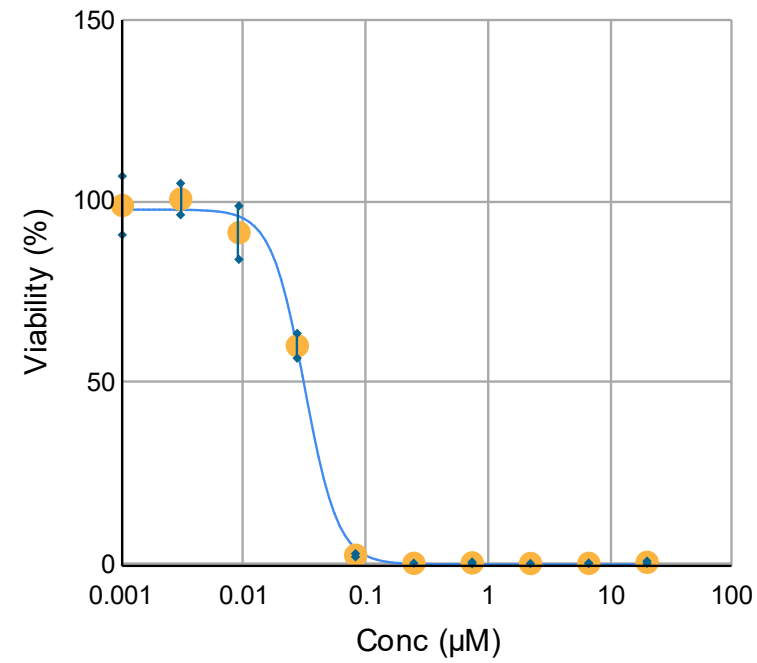


Synthetic 1a (proposed)

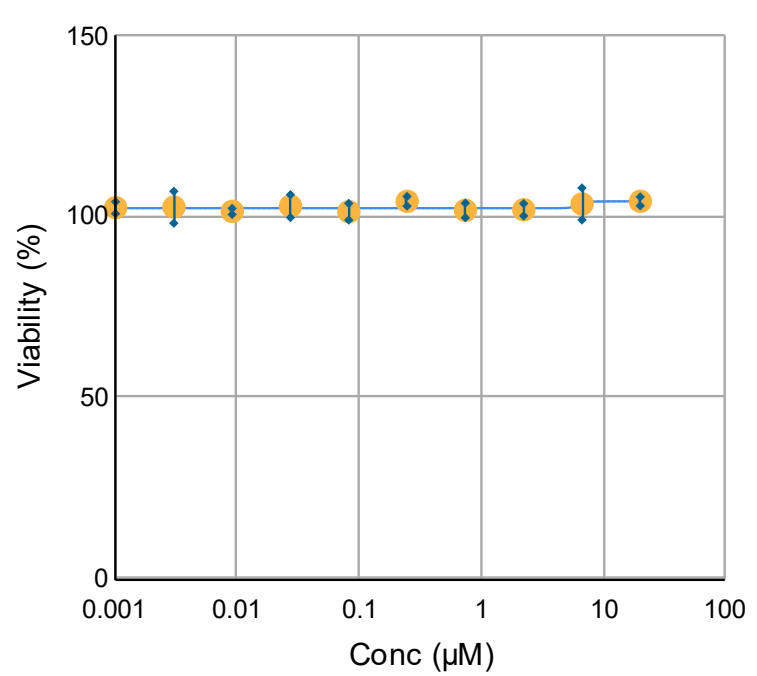

Synthetic 1b (revised)

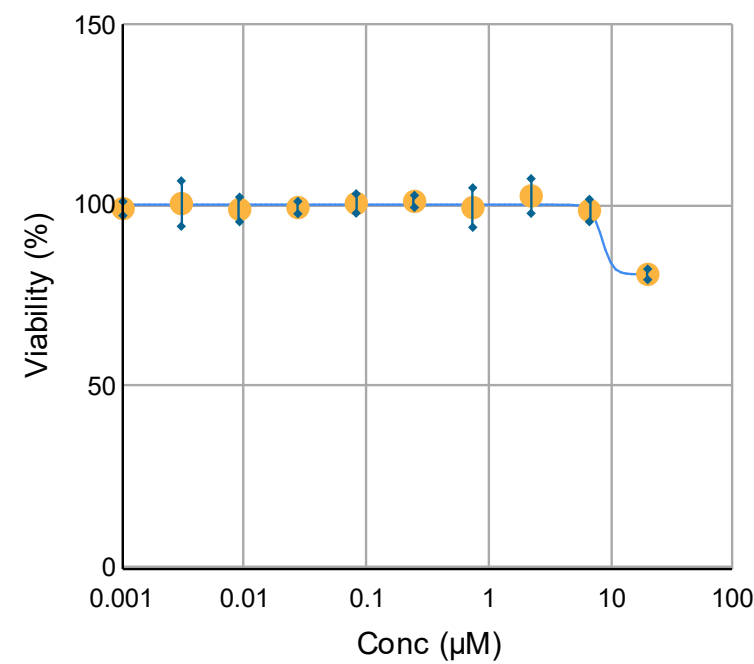

Mitomicyn C

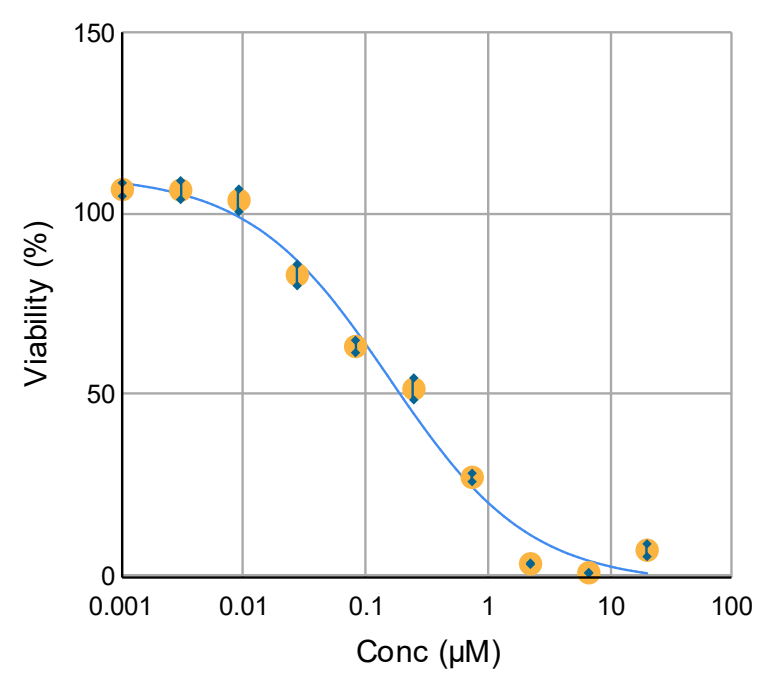

